\title{
LOW-FREQUENCY SERIES LOADED RESONANT INVERTER CHARACTERIZATION
}

\author{
A Thesis \\ presented to \\ the Faculty of California Polytechnic State University, \\ San Luis Obispo
}

\author{
In Partial Fulfillment \\ of the Requirements for the Degree \\ Master of Science in Electrical Engineering
}

by

Alfredo Medina

June 2016 
(C)2016

Alfredo Medina

ALL RIGHTS RESERVED 


\section{COMMITTEE MEMBERSHIP}

TITLE: Low-Frequency Series Loaded Resonant Inverter

Characterization

AUTHOR: Alfredo Medina

DATE SUBMITTED: June, 2016

COMMITTEE CHAIR: Taufik, Ph.D.

Professor of Electrical Engineering

COMMITTEE MEMBER: Ali O. Shaban, Ph.D.

Professor of Electrical Engineering

COMMITTEE MEMBER: Ahmad Nafisi, Ph.D.

Professor of Electrical Engineering 


\section{ABSTRACT \\ Low-Frequency Series Loaded Resonant Inverter Characterization \\ Alfredo Medina}

Modern power systems require multiple conversions between DC and AC to deliver power from renewable energy sources. Recent growth in DC loads result in increased system costs and reduced efficiency, due to redundant conversions. Advances in DC microgrid systems demonstrate superior performance by reducing conversion stages. The literature reveals practical DC microgrid systems composed of wind and solar power to replace existing fossil fuel technologies for residential consumers. Although higher efficiencies are achieved, some household appliances require AC power; thus, the need for highly efficient DC to AC converters is imperative in establishing DC microgrid systems. Resonant inverter topologies exhibit zero current switching (ZCS); hence, eliminate switching losses leading to higher efficiencies in comparison to hard switched topologies.

Resonant inverters suffer severe limitations mainly attributed to a load dependent resonant frequency. Recent advancements in power electronics propose an electronically tunable inductor suited for low frequency applications [24], [25]; as a consequence, frequency stability in resonant inverters is achievable within a limited load range. This thesis characterizes the operational characteristics of a low-frequency series loaded resonant inverter using a manually tunable inductor to achieve frequency stability and determine feasibility of utilization. Simulation and hardware results demonstrate elimination of switching losses via ZCS; however, significant losses are observed in the resonant inductor which compromises overall system efficiency. Additionally, harmonic distortion severely impacts output power quality and limits practical applications.

Keywords: Resonant Inverter, Low-Frequency Resonance, Series Resonance, ZCS Inverter 


\section{ACKNOWLEDGMENTS}

En la búsqueda de la sabiduría siempre es importante tener personas dispuestas a apoyarte. Esas personas merecen más de lo que puedo dar en esta vida, ya que sin ellas no podría haber llegado tan lejos en mi camino. Son tantas personas que me han apoyado en mi vida, que es difícil nombrarlas a todas. Pero sin más que decir, es claro que las personas que me han apoyado con todo su amor y enseñanzas son mis queridos padres, Ramón y María Medina. Mis padres son mis primeros educadores y modelos a seguir. Sin ellos, no puedo estar seguro que tendría esta vida feliz. Con su apoyo, me he transformado en una persona completa y optimista. Lo que he aprendido de cada uno de ellos es único. Ramón me enseñó como ser hombre; como proteger,

proveer, y trabajar. Los días que mi padre me llevó a trabajar con él, son algunos de los recuerdos más felices para mí. María me enseñó como amar, apreciar, y mostrar afecto. Recuerdo los días en que mi madre venía a casa del trabajo y sus ojos se iluminaban de alegría al ver a sus hijos. Por esas enseñanzas y experiencias les quiero dar gracias a mis padres. Ahora enfrento al mundo con confianza de perseguir mis sueños y combatir cualquier obstáculo. 


\section{TABLE OF CONTENTS}

Page

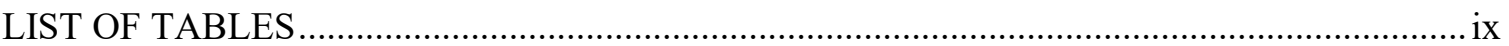

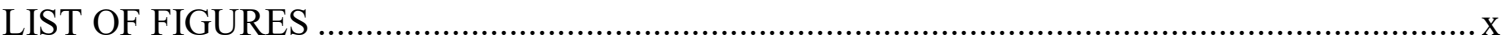

CHAPTER

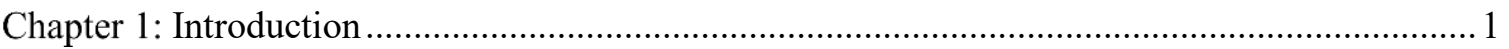

1.1. Incentive

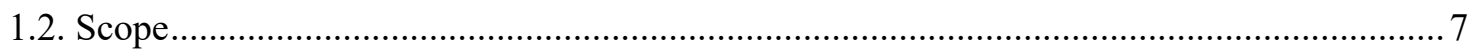

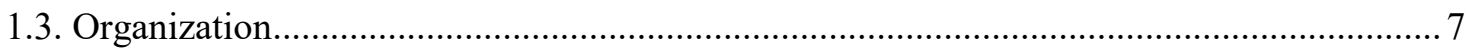

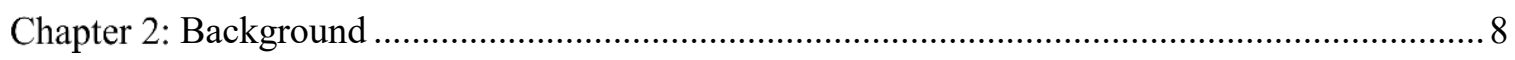

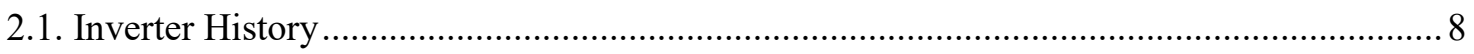

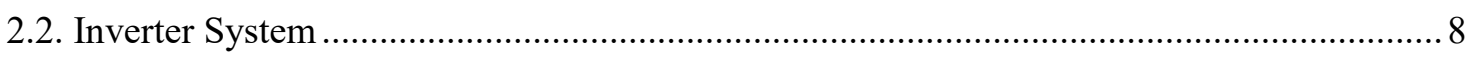

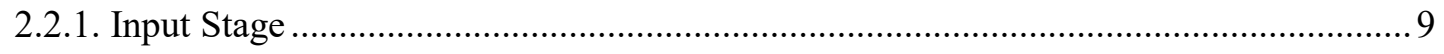

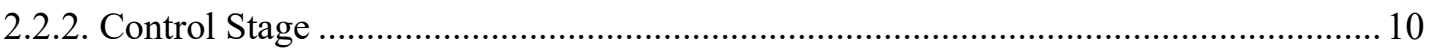

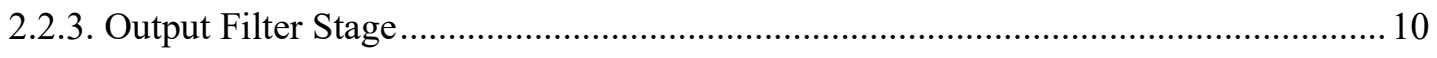

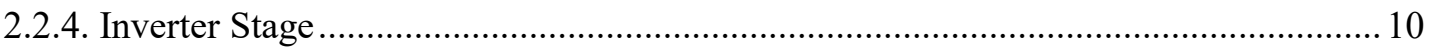

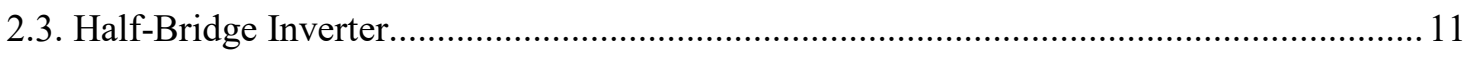

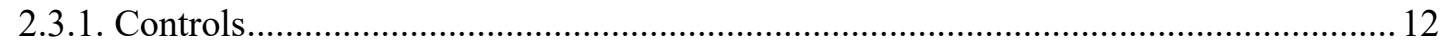

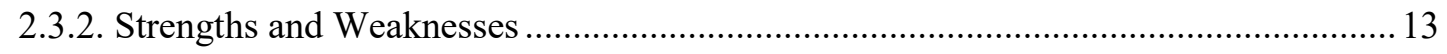

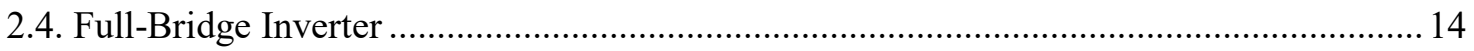

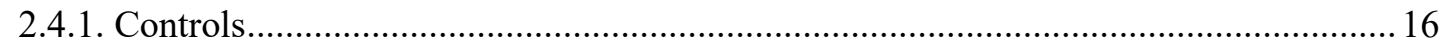

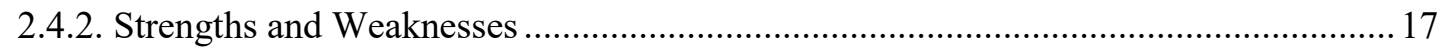

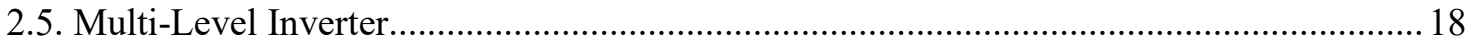

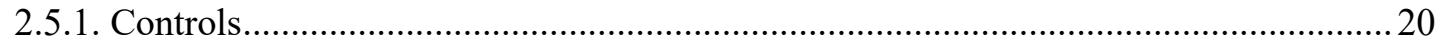

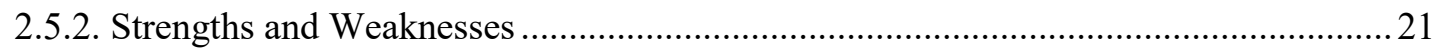

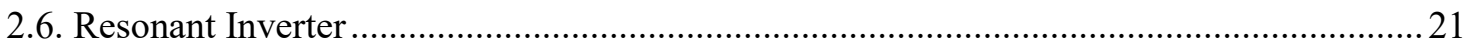




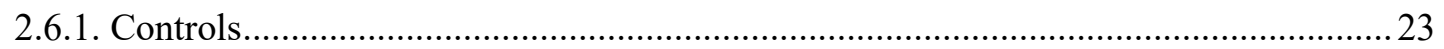

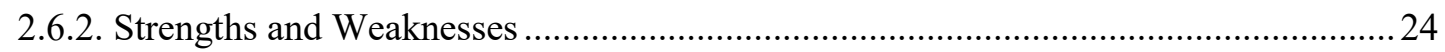

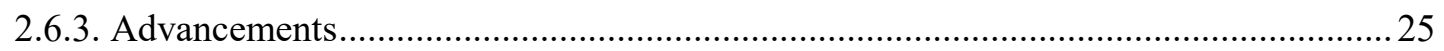

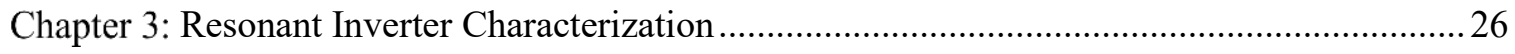

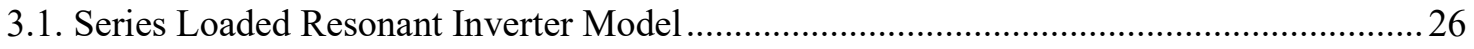

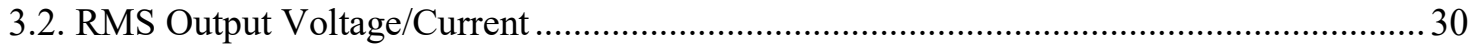

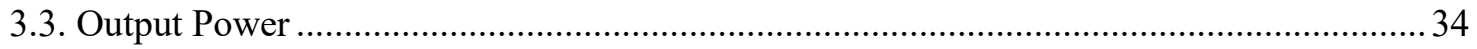

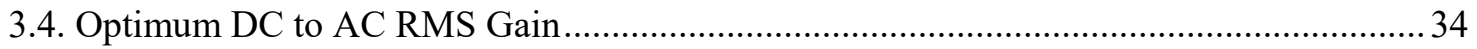

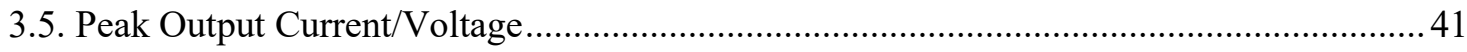

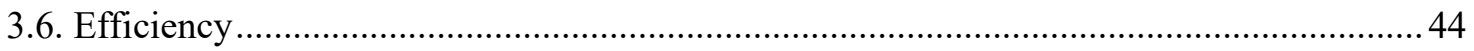

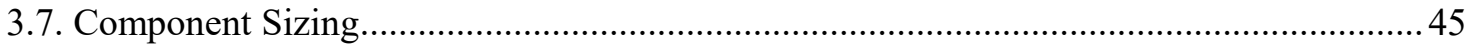

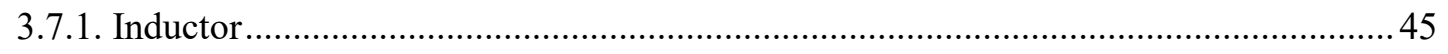

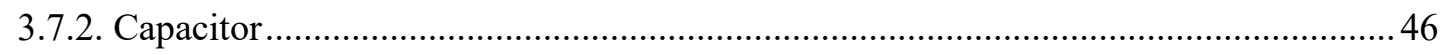

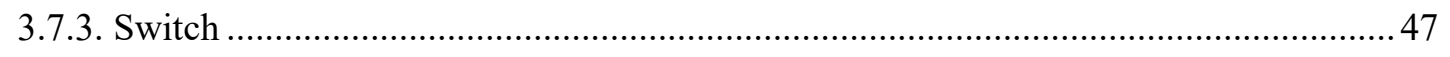

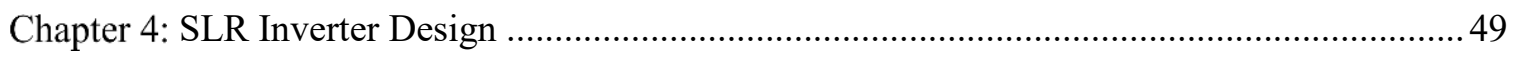

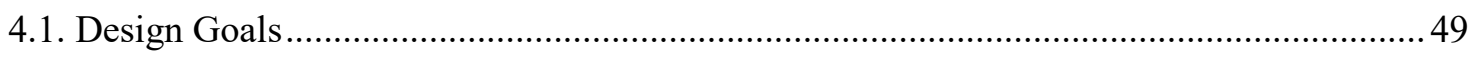

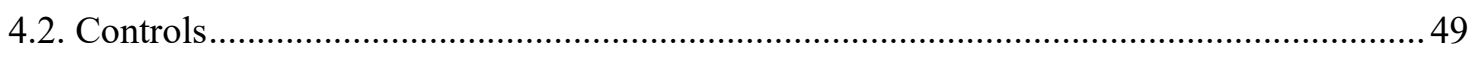

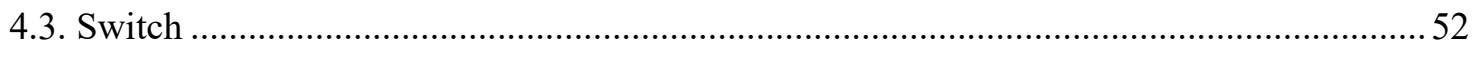

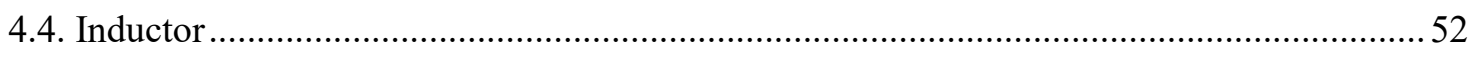

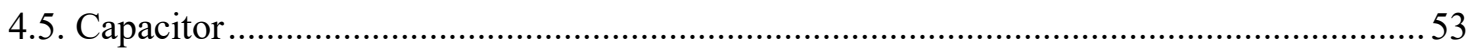

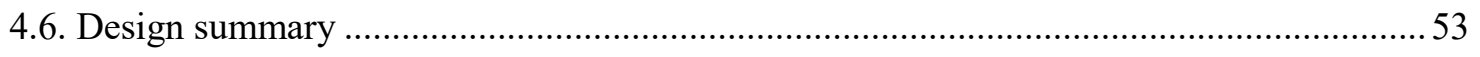

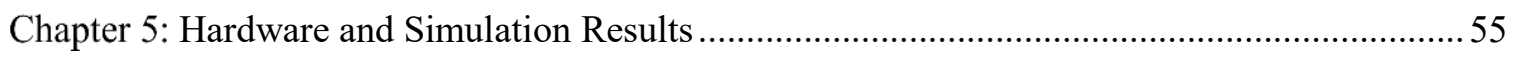

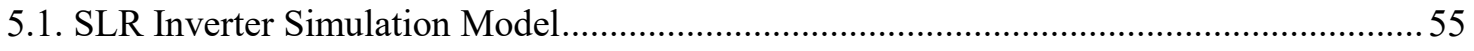

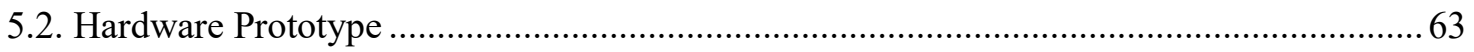

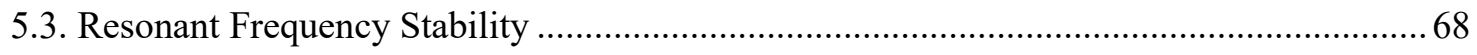

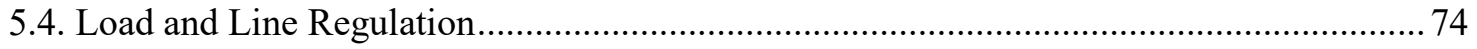




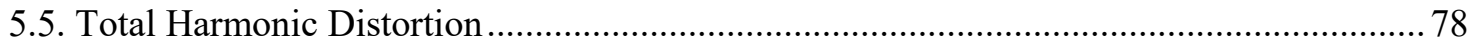

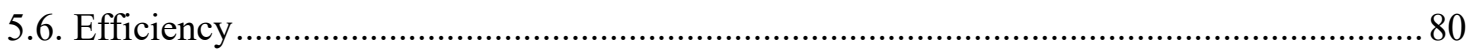

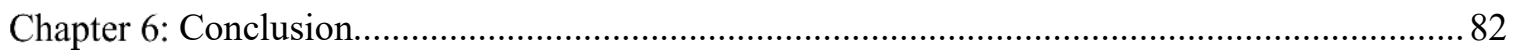

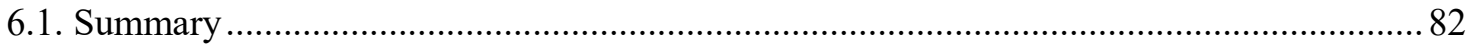

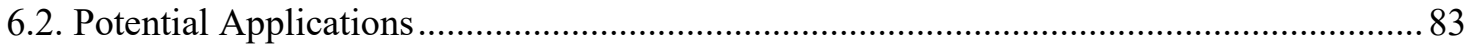

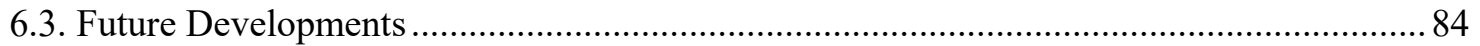

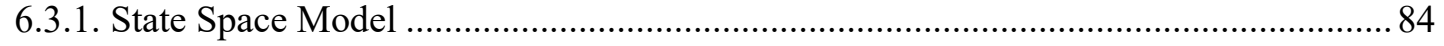

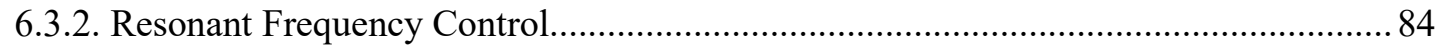

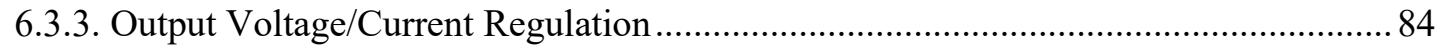

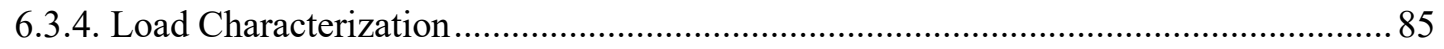

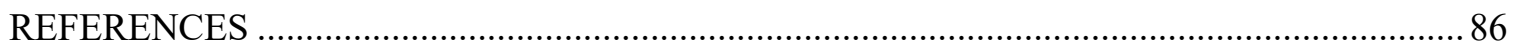

Appendix A: LTspice Half-Bridge Controller Module............................................................... 90 


\section{LIST OF TABLES}

Table

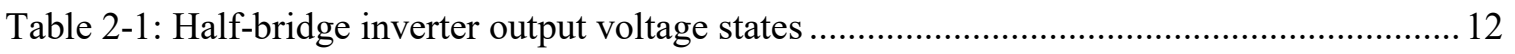

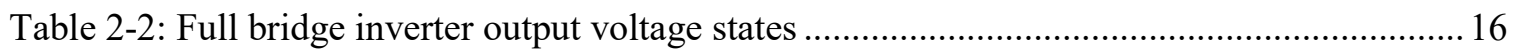

Table 2-3: Three level multi-level inverter output voltage states ............................................... 19

Table 2-4: Series loaded resonant inverter output voltage states ...............................................23

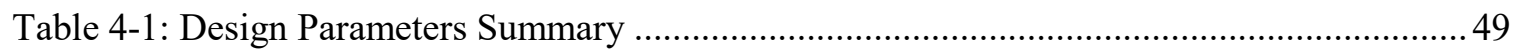

Table 4-2: IRS2153 self-oscillating controller design values ...................................................51

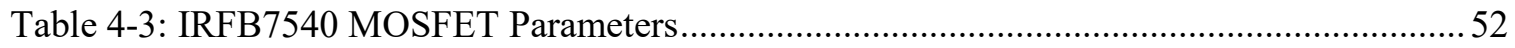

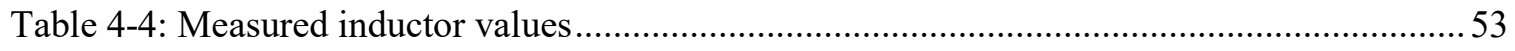

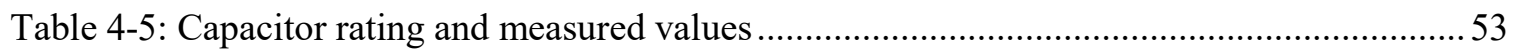

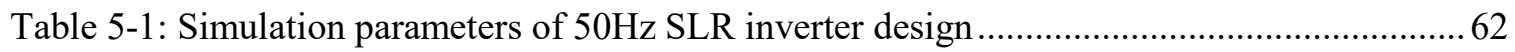

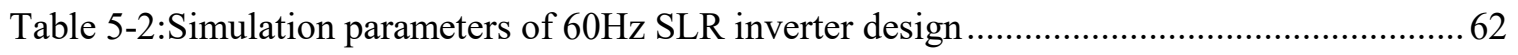

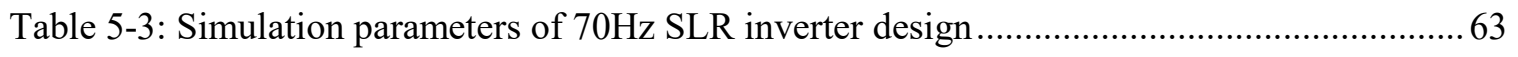

Table 5-4: SLR inverter prototype design vs. measurement...................................................... 65

Table 5-5: General Radio 940-E variable inductor measured parameters ...................................... 68 


\section{LIST OF FIGURES}

Figure

Page

Figure 1-1: 1973 and 2013 world fuel shares of total primary energy supply, courtesy of [1] ......2

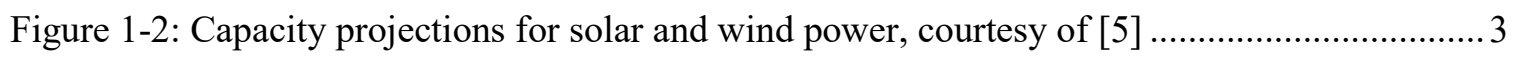

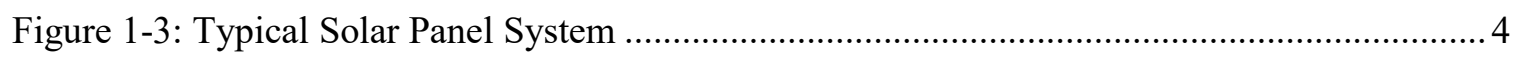

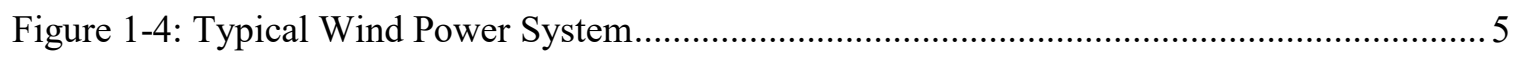

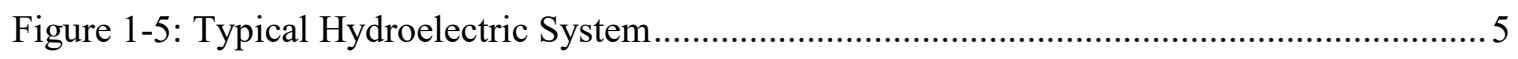

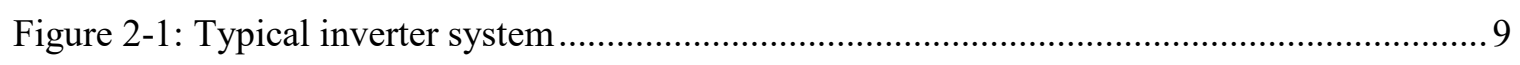

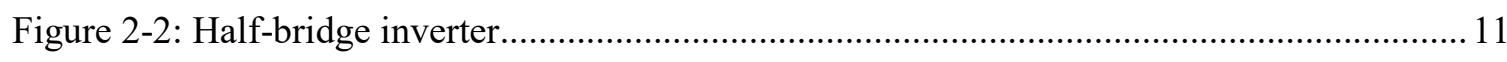

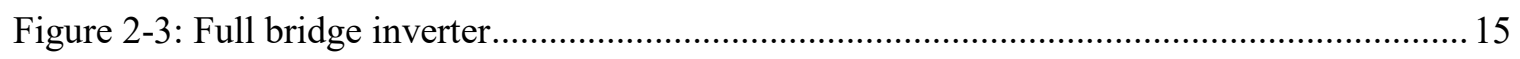

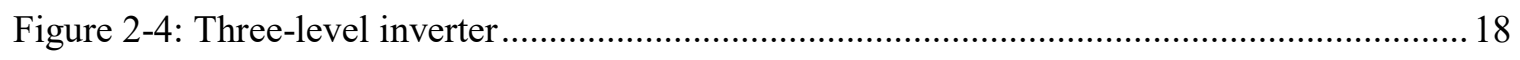

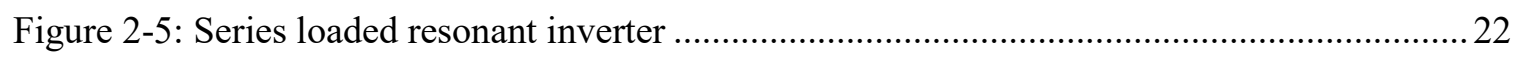

Figure 3-1: Frequency domain representation of output RLC network ...................................26

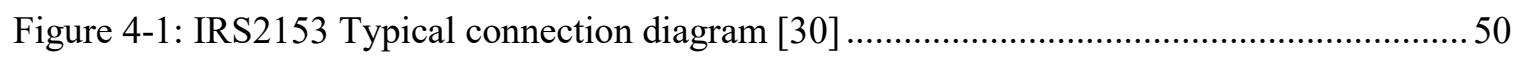

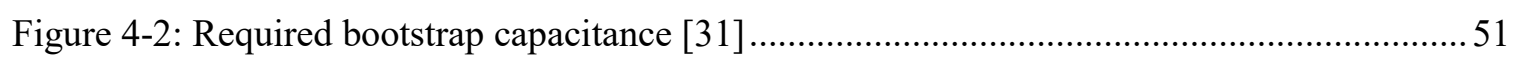

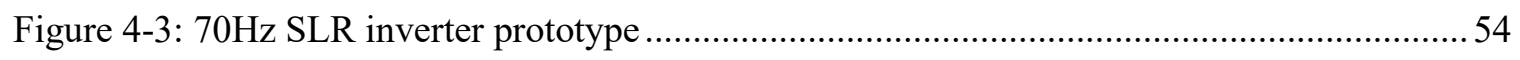

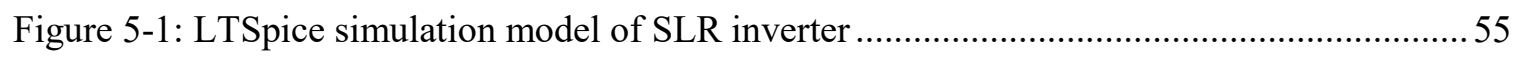

Figure 5-2: SLR inverter switch current and voltage demonstrating ZCS ..................................56

Figure 5-3: Capacitor voltage, switch node voltage, and output current demonstrating capacitor

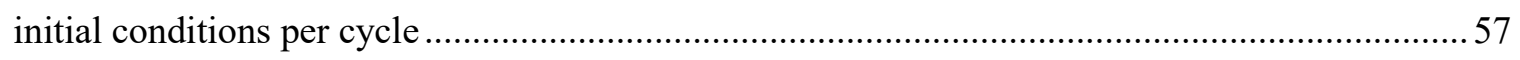

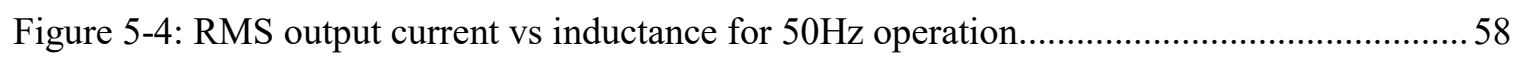

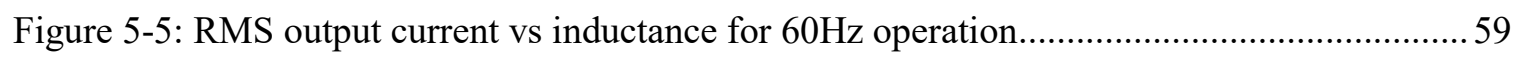

Figure 5-6: RMS output current vs inductance for $70 \mathrm{~Hz}$ operation............................................59

Figure 5-7: Percent error between calculated and steady state RMS output voltage ....................60

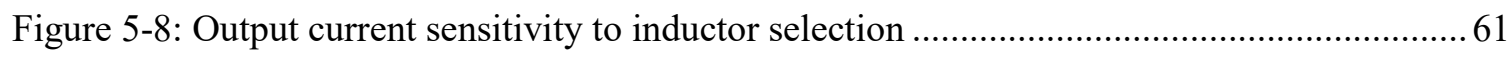

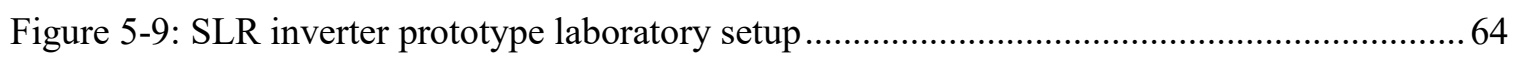




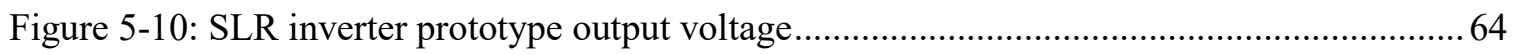

Figure 5-11: SLR inverter prototype switch node (SW) and output voltage (Vout)..................... 66

Figure 5-12: SLR inverter prototype switch node (SW), and capacitor voltage (Vcap)...............66

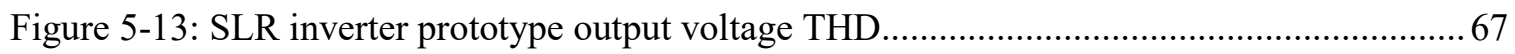

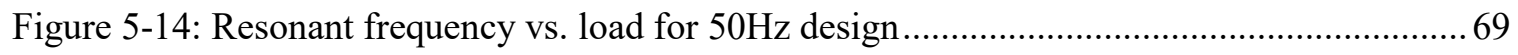

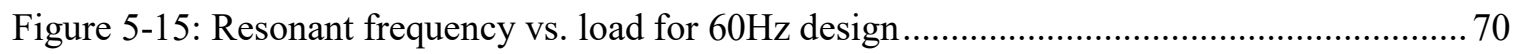

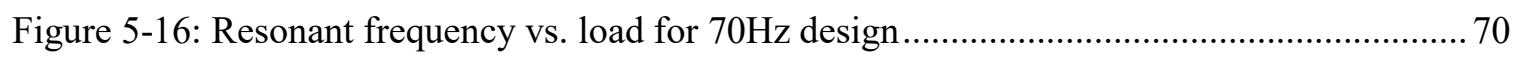

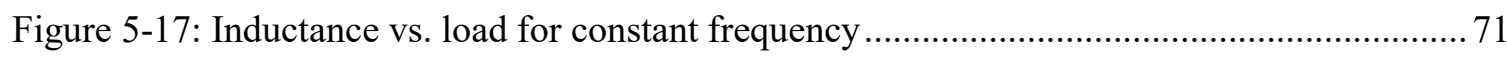

Figure 5-18: Frequency stability using variable inductance hardware results ............................. 74

Figure 5-19: Load and line regulation for $50 \mathrm{~Hz}$ design using variable inductance frequency

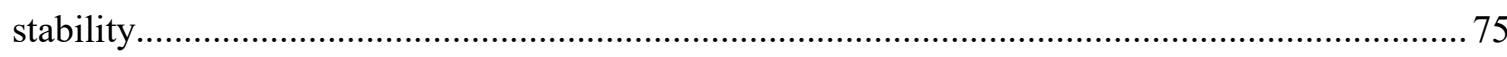

Figure 5-20: Load and line regulation for $60 \mathrm{~Hz}$ design using variable inductance frequency

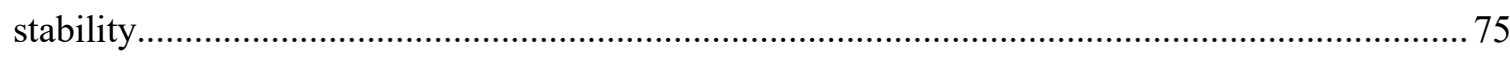

Figure 5-21: Load and line regulation for $70 \mathrm{~Hz}$ design using variable inductance frequency

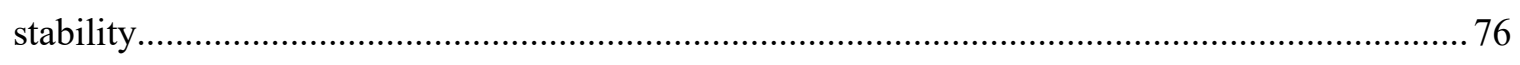

Figure 5-22: Current regulation using variable inductance hardware results .............................. 77

Figure 5-23: Output current THD vs. load for constant frequency using variable inductance .......78

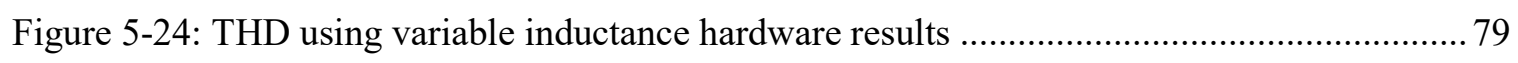

Figure 5-25: Efficiency vs. load resistance for constant frequency using variable inductance ..... 80

Figure 5-26: Hardware efficiency using variable inductance frequency stability ........................ 81 


\section{Chapter 1: Introduction}

\subsection{Incentive}

Renewable energy has set the tone for the early $21^{\text {st }}$ century. A consequence of Earth's pollution, scarcity of resources, and overpopulation; all of which are human induced factors. At the heart of these issues is energy. Energy is the source of all matter and life in the universe; therefore, being conscious of the supply and use of energy is detrimental for the future of humanity. Now more than ever, researchers, scientists, and engineers are pushing for highly efficient self-sustainable systems to combat these issues.

Crude oil, coal, and natural gas have historically supplied a majority of the world's energy, as shown in Figure 1-1. These energy supplies are limited, emit pollutants, and are linked to global climate change [2]. Nuclear and bioenergy systems seem promising, but exhibit issues such as resource limitations, water use, land use, and have a considerable risk of catastrophe [3]. Research conducted by [3] proposes a completely self-sustainable energy system incorporating wind, water, and solar (WWS) power as early as 2050. The benefits of a WWS system extend to all facets of our society and include: low pollution, low cost, and a zero catastrophe risk [3]. While the technology for using renewable resources is available, [3] argues that "The obstacles to powering the world with wind, water, and sunshine are primarily social and political, not technical or economic". 


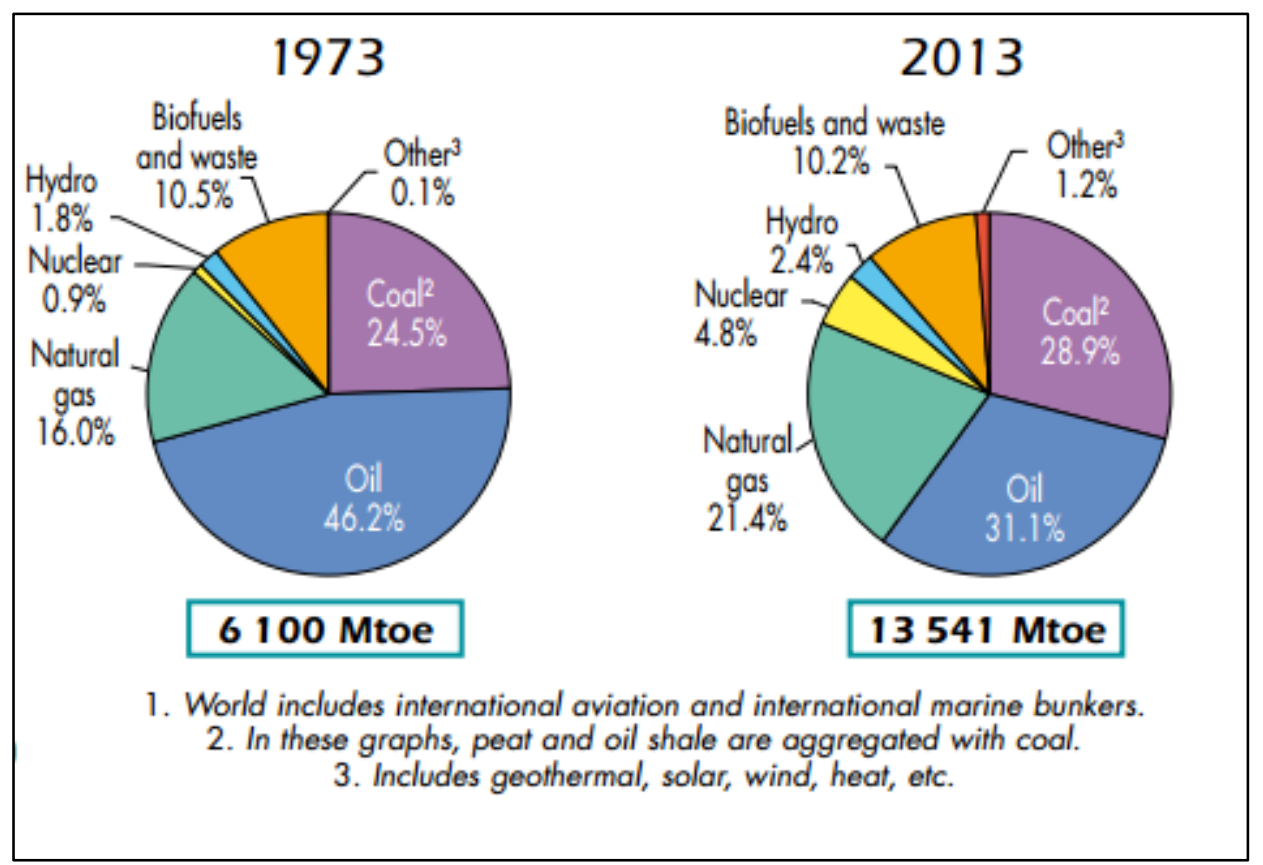

Figure 1-1: 1973 and 2013 world fuel shares of total primary energy supply, courtesy of [1]

Policymaking plays a crucial role in promoting renewable energy sources. Policies supporting renewable energies, such as the recently adopted U.S. Clean Power Plan, are already under work. The Clean Power Plan establishes emission guidelines for existing fossil fuel powered electric generating units [4]. The guidelines limit existing power plant $\mathrm{CO}_{2}$ emissions and support renewable energy for both the generation and load side. These government policies are critical for the advancement of renewable energies. Figure 1-2 demonstrates capacity projections for solar and wind generation in the annual energy outlook (AEO) of 2015 with and without the proposed Clean Power Plan. Note that with the proposed Clean Power Plan, solar and wind capacity in the year 2040 is expected to be $125 \%$ and $87 \%$, respectively, greater than the reference case, which does not include the Clean Power Plan. Though these energies demonstrate a promising future on the supply side, an emphasis must also be placed on the electronics that govern generation, transmission, and distribution of renewable energy in order to yield high system efficiencies. 


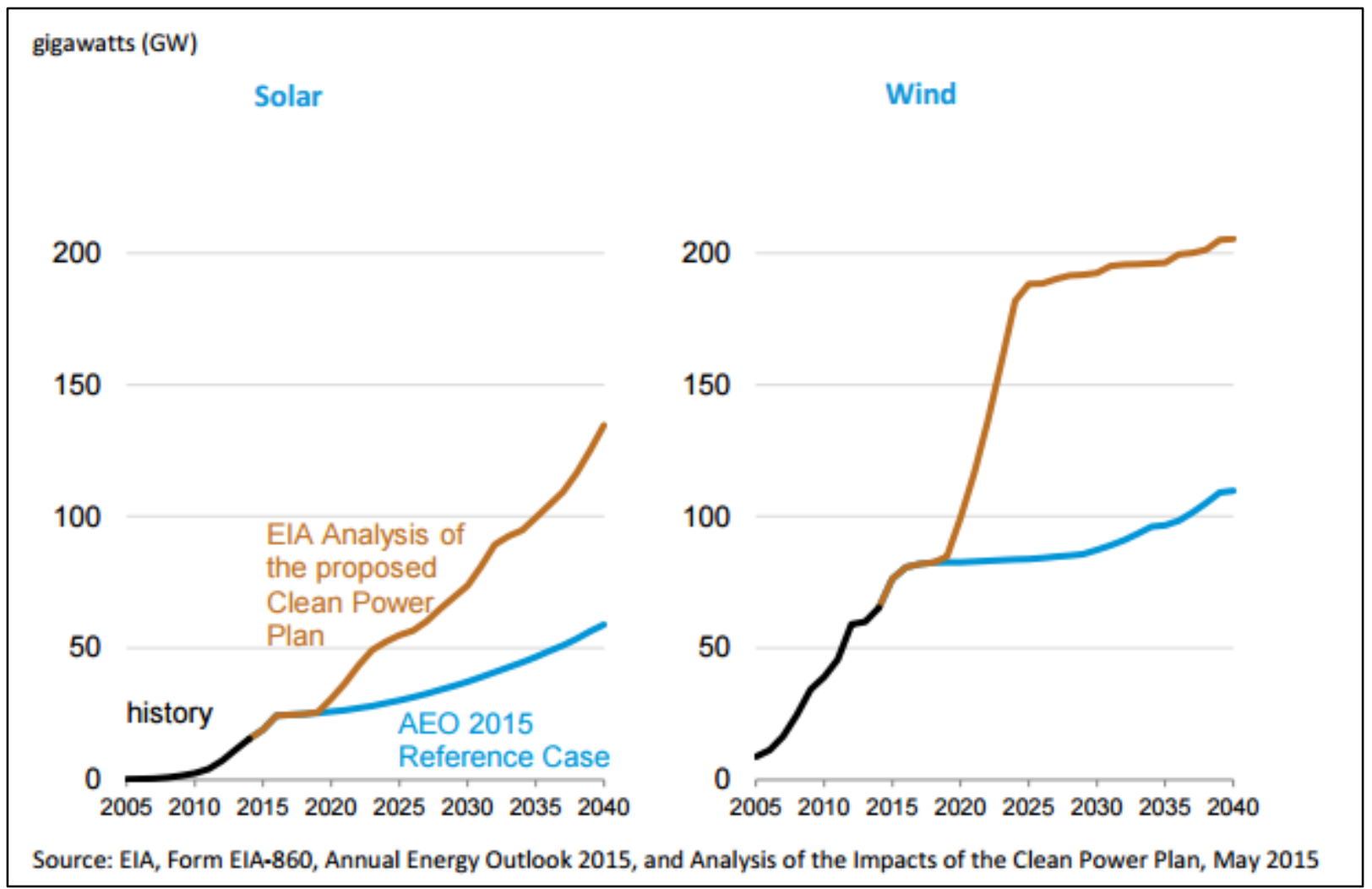

Figure 1-2: Capacity projections for solar and wind power, courtesy of [5]

To understand the electronic requirements for WWS power, it is important to analyze the systems that govern the generation of electricity from renewable energy sources. Figure 1-3 demonstrates a typical solar panel system. Solar panel systems produce DC power via the photovoltaic effect and use DC-DC converters to store energy in a battery bank. Figure 1-4 demonstrates a typical wind power system. Wind power systems convert the mechanical energy of a rotating turbine to $\mathrm{AC}$ power via a generator. The rotation of the turbine is caused by wind current. The generated AC power is unpredictable and non-dispatchable, due to wind fluctuations; thus, an AC-DC converter is required to rectify AC power to store in a battery bank. Figure 1-5 demonstrates a typical hydroelectric system. Hydroelectric systems also use a turbine to turn mechanical energy into AC power; however, the rotation of the turbine is caused by water current. Water power is inherently an uncontrollable energy source; though, dams are often used to regulate the amount of water flow and thus the amount of generated AC power. Note that the 
hydroelectric dam is a dispatchable generating unit, thus may be connected directly to the grid. For the case of wind and solar, an inverter must be introduced in order to deliver energy to the AC grid. The need for multiple power conversions in wind and solar systems undesirably impacts the overall efficiency of the system. The efficiency is impacted even more when the AC power must be rectified to power DC devices such as laptops, cell phones, computers, etc. This proposes a problem between the current $\mathrm{AC}$ distribution system and the sought after renewable energy.

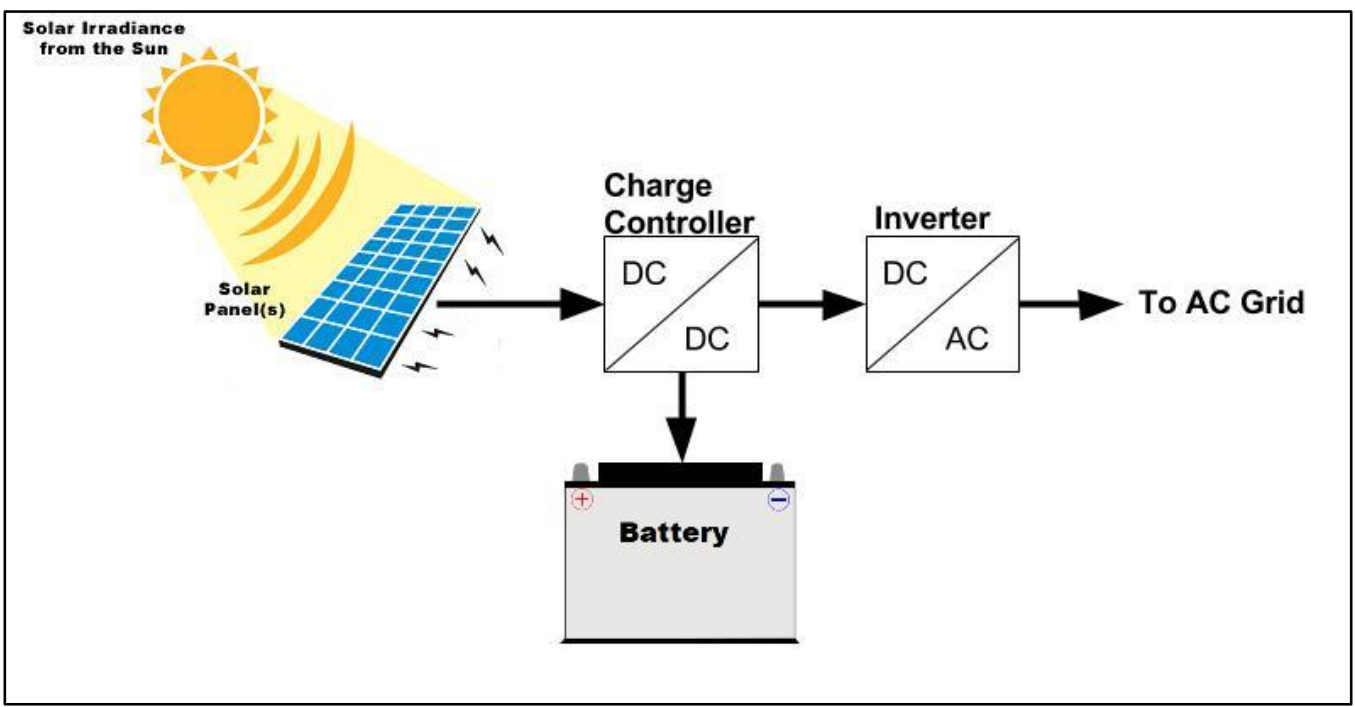

Figure 1-3: Typical Solar Panel System 


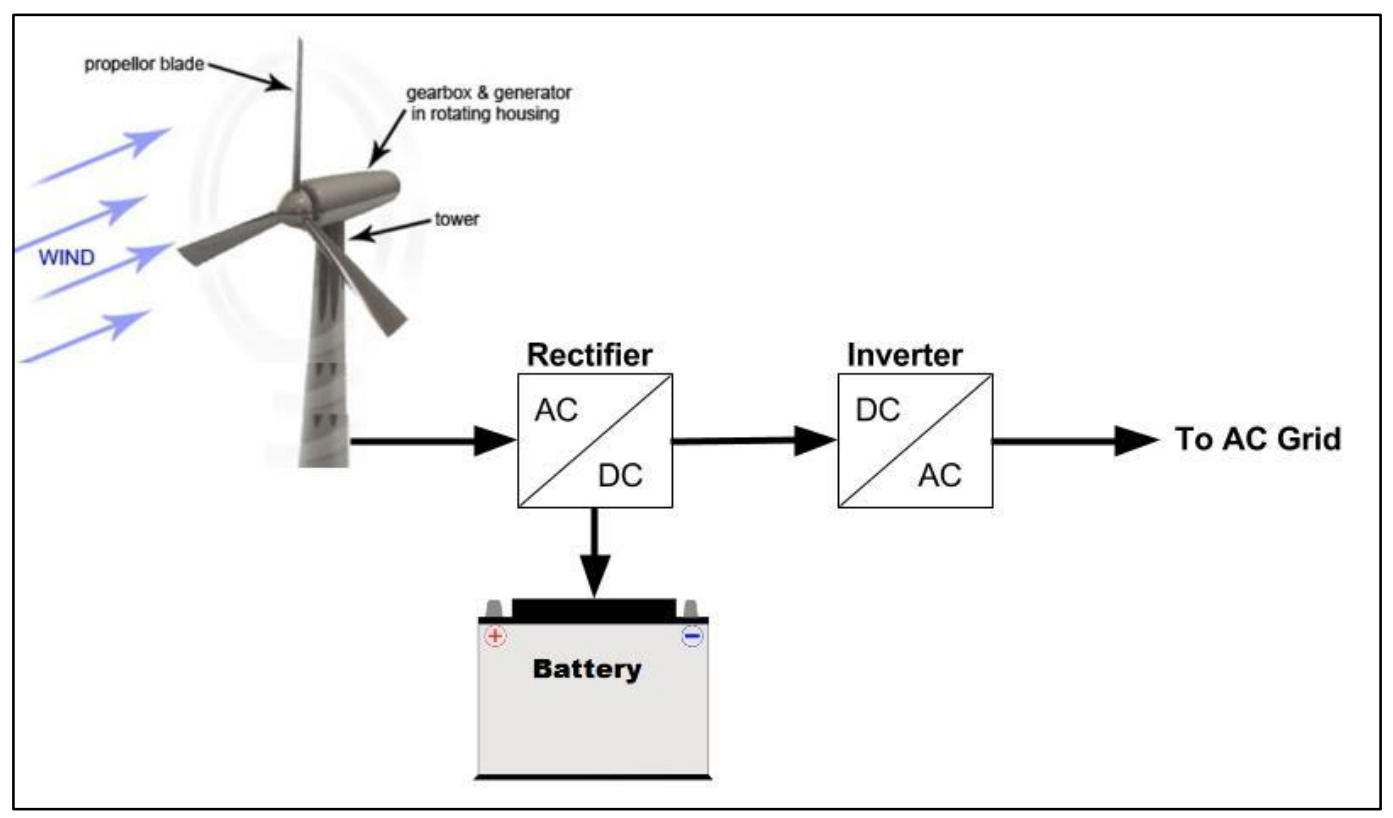

Figure 1-4: Typical Wind Power System

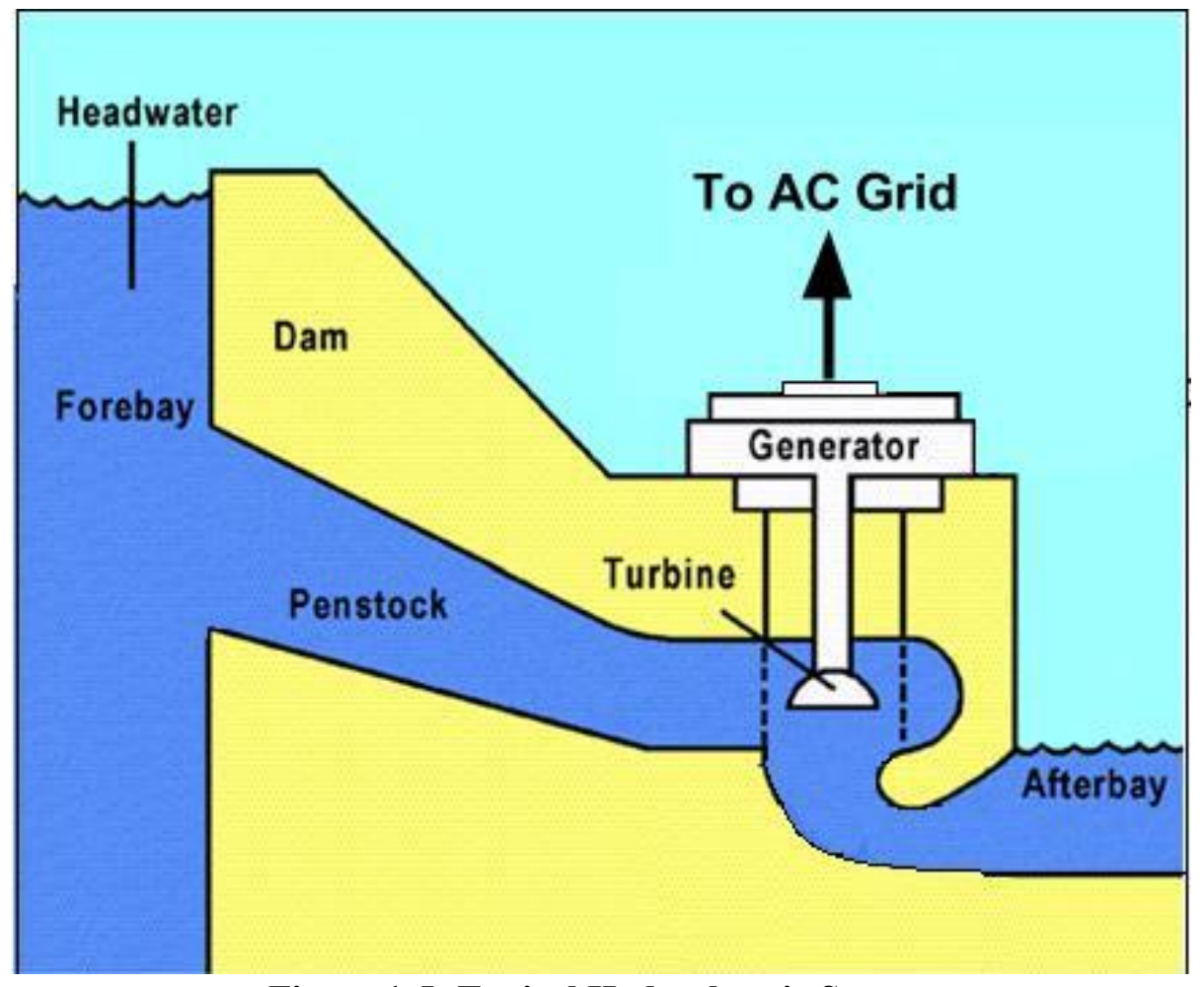

Figure 1-5: Typical Hydroelectric System

Recent advancements propose DC microgrid systems to combat the adverse effects of multiple power conversions [6]-[9]. Microgrid systems may be composed of distributed energy sources, such as the method proposed in [3], thus supporting WWS systems. Microgrids may be 
designed as $\mathrm{AC}, \mathrm{DC}$, or hybrid $\mathrm{AC} / \mathrm{DC}$ systems. In [6], $\mathrm{AC}$ and $\mathrm{DC}$ microgrids are compared and results show $15 \%$ less losses in the DC microgrid; however, the study was conducted with only DC loads. [7] also compares the AC and DC microgrids with an emphasis on economics and demonstrates that "DC microgrids could potentially improve microgrid economic benefits when the ratio of DC loads is high" [7]. The increasing demand of DC loads is described in [8] which suggests that high distortion loads be converted to a DC system to increase efficiency and decrease harmonic injection onto the $\mathrm{AC}$ grid. Though there is an increasing demand for $\mathrm{DC}$ loads, an AC system is still required to incorporate dispatchable generating units to meet a high energy demand when DC energy sources do not suffice [9].

Although the generation and transmission of renewable energy sources may be accomplished via DC, there exists the need for AC power in various household applications. Household appliances requiring $\mathrm{AC}$ power include: fans, induction cooktops, compact fluorescent lights (CFL), air conditioners, refrigerators, microwaves, etc.; thus, the need for DC to AC conversion is necessary. The power requirements for these appliances may be met with the use of highly efficient point of load inverters. Current inverter technologies utilize switching topologies; however, this creates harmonics and reduces inverter efficiency. Resonant inverters eliminate switching losses by using resonance to accomplish DC to AC conversion. In addition, resonant inverters are highly efficient, since losses only occur in the parasitic resistance of components. However, the performance characteristics are highly dependent on the load and very little research has been conducted in this field. This thesis aims to design and analyze a $60 \mathrm{~Hz}$ resonant inverter based on a maximum DC to AC voltage gain; thus, quantifying operating characteristics to determine feasibility of utilization. 


\subsection{Scope}

The scope of this thesis is limited to the analysis and simulation of a low frequency series loaded resonant (SLR) inverter. The author uses a transfer function method to develop a mathematical model of the SLR inverter and proposes a design strategy for maximum DC to AC gain. The load dependent performance characteristics of the SLR inverter are analyzed using the derived mathematical model. These performance characteristics include: resonant frequency, output voltage, efficiency, and quality factor. The model assumes low frequency operation and does not account for high frequency parasitic components. In addition, the model assumes a purely resistive load and does not account for leading or lagging loads. The developed model is compared to hardware and simulation via LTSpice.

\subsection{Organization}

The following thesis chapter presents an introduction to inverters, followed by survey on existing inverter topologies, and ends with a justification of the proposed SLR inverter topology. Chapter 3 establishes the requirements of the proposed SLR inverter. Chapter 4 forms the basis of the thesis, and focuses on the analysis and simulation of the proposed SLR inverter. Chapter 5 compares and analyzes results from simulation and the derived model. This thesis ends in chapter 6 with a summary of the performed work, recommendation of potential SLR inverter applications, and recommendation of future SLR inverter developments. 


\section{Chapter 2: Background}

\subsection{Inverter History}

The idea of controlling current flow using gates in conjunction with phase delay to modulate AC power was first proposed in the early 1920's [11]. Those that proposed this new idea are not known; however, in 1925 David Prince wrote an article titled "The Inverter" and is credited for establishing the term "inverter", as cited in [11]. Initial inverter technologies incorporated mechanical commutation and vacuum tube devices [11]. Mechanical commutation inverters (Rotary Inverters) use the rotation of a motor to route DC to an AC load. Vacuum tube inverters use vacuum tube devices as valves to direct the flow of current. These inverters were eventually deemed obsolete, due to the rise of semi-conductor technology which yielded higher efficiencies and controllability. In present day, the majority of inverters use semi-conductor technology to convert DC to AC.

\subsection{Inverter System}

Modern day inverters use semi-conductor technology to route electrical energy via switching and forms the foundation of inverter functionality. Though all inverters use semiconductor switches, inverter systems differ significantly in performance, cost, and size. The typical inverter system is shown in Figure 2-1. Note that in addition to the inverter stage, an input power conditioning stage, control stage, and output filtering stage are used to increase system performance and reliability. Although this thesis focuses on the inverter power stage, it is imperative that the reader understand the significance of each stage in the inverter system. 


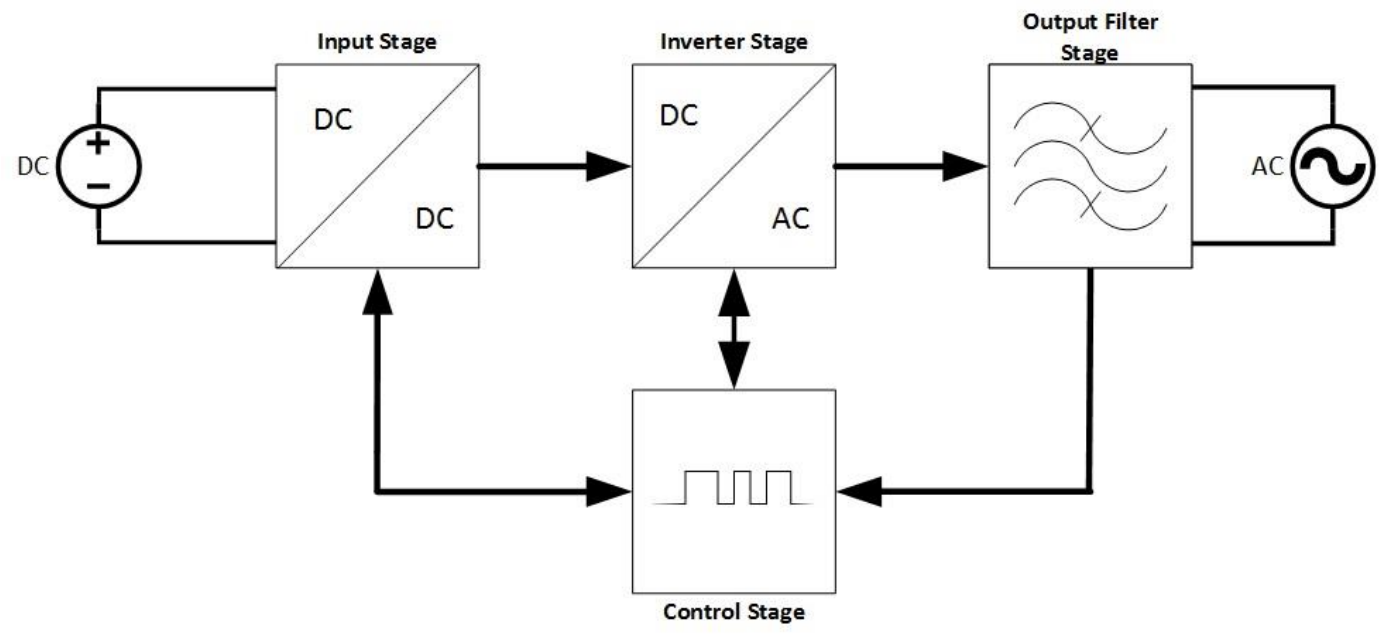

Figure 2-1: Typical inverter system

\subsubsection{Input Stage}

The input stage is composed of a DC-DC converter used to regulate the DC bus voltage supplied to the inverter stage. This stage is commonly used in solar power inverters to draw the maximum amount of power from a solar panel, known as maximum power point tracking (MPPT) [12]. In addition to MPPT, the input stage reduces the low frequency current ripple current generated by the inverter stage; thus, reducing the distortion effecting the grid.

Alternately, the input may be an $\mathrm{AC}$ source such as the case with variable speed drives. In the occurrence of an AC source, the input stage is composed of an AC-DC converter, also known as a rectifier. Variable speed drives augment the frequency of the AC source to drive electronic motors [13]. For variable speed drives, the input stage must both rectify and regulate the DC bus voltage of the inverter stage. Rectification contributes distortion to the line power, in addition to the distortion generated by the inverter stage; thus complicating the filtering task of the input stage. 


\subsubsection{Control Stage}

The control stage generates electrical signals that trigger the inverter stage switches. Furthermore, feedback in the control stage regulates system parameters including temperature, output voltage, and output frequency. It is important to note that extensive research exists in the field of inverter controls, thus only a few of the most commonly regulated parameters are mentioned. Control systems vary in complexity and size depending on the application and fidelity of the system.

\subsubsection{Output Filter Stage}

The output filtering stage attenuates high frequency harmonics generated by switching in the inverter stage. Typically, the inverter stage outputs a desired low frequency and an undesirable high switching frequency. The responsibility of the output filter is to attenuate the harmonics of the high switching frequency. The resulting frequency content of the output is the fundamental component of the low frequency signal. These filters are typically exhibit either low-pass or band-pass frequency response.

\subsubsection{Inverter Stage}

The inverter stage is composed of an essential topology used to convert DC to AC and is the focus of this thesis. Issues with the inverter power stage include input current ripple, output current/voltage distortion, and efficiency. The input current ripple is a result of the output $\mathrm{AC}$ power demand causing distortion of the line current and may be detrimental to electro-magnetic compliance (EMC). These distortions are typically handled by the input stage. Output current and voltage distortion is caused by the harmonics associated with the switching frequency. These harmonics reduce efficiency and compromise the stability of sensitive loads. Overall, the inverter stage must diminish switching harmonics while maintaining a high efficiency. 


\subsection{Half-Bridge Inverter}

The half-bridge inverter is the most basic circuit for converting DC to AC. The topology uses a minimum of 2 switches to accomplish inverter functionality. The half-bridge inverter is only capable of generating two output voltage states, which correspond to the positive and negative rails of the input DC voltages. The half-bridge inverter is shown in Figure 2-2.

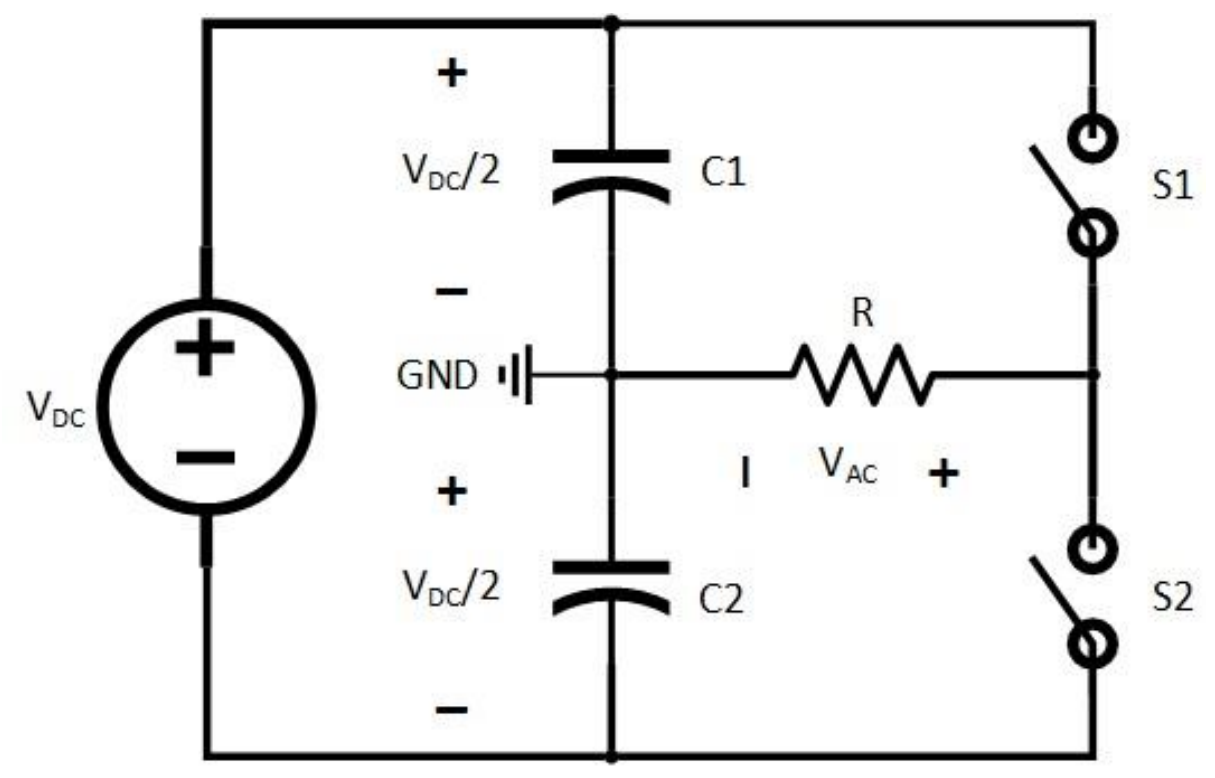

Figure 2-2: Half-bridge inverter

When SW1 is on and SW2 is off, a positive voltage is applied to the load. When SW1 is off and SW2 is on, a negative voltage is applied to the load. When both switches are off the voltage across a purely resistive load is $0 \mathrm{~V}$; however, if the load is either capacitive or inductive the output voltage state is unknown. The unknown voltage state is not used, since it cannot be defined. When both switches are on, a short is created between the terminals of the DC input voltage. Both switches are ensured to never be on at the same time to avoid shorting the supply. The practical states of the half-bridge are summarized in Table 2-1. This forms the basis of the operation for the half-bridge inverter. A brief discussion on control strategies encompasses the performances of the half-bridge topology. 
Table 2-1: Half-bridge inverter output voltage states

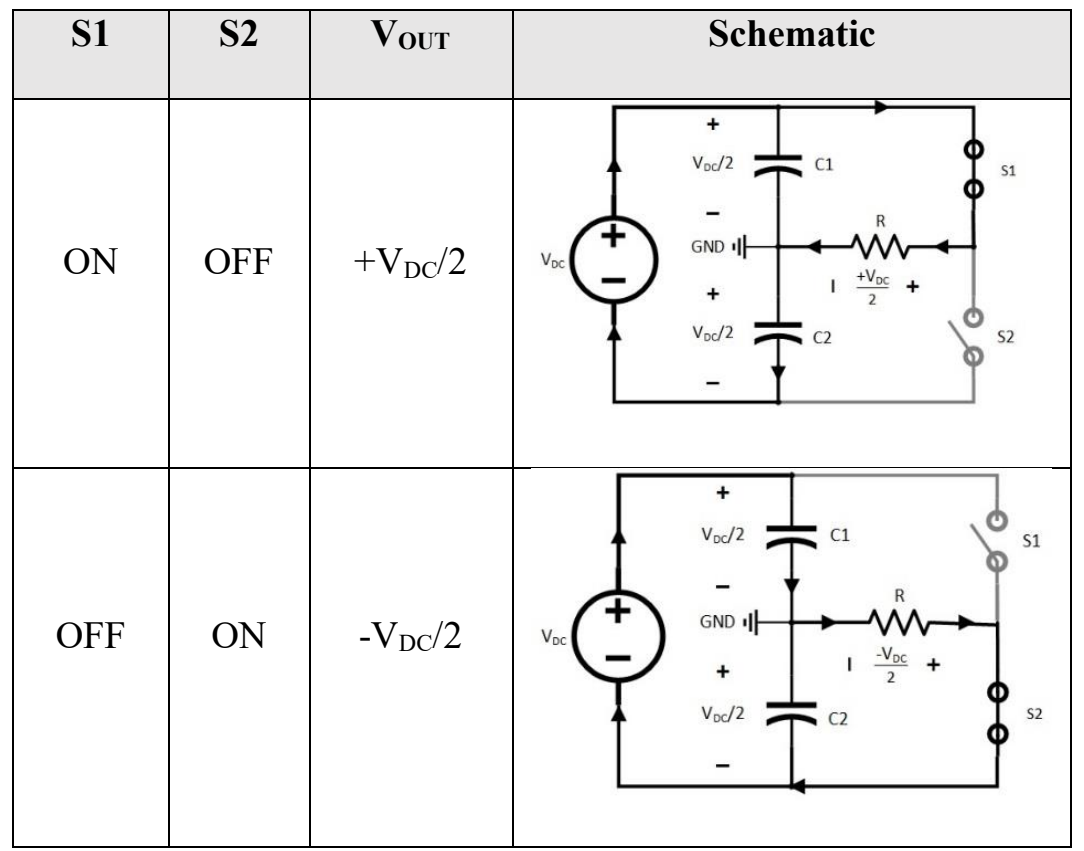

\subsubsection{Controls}

Control strategies for the half bridge inverter are limited by the available output voltage states. A simple strategy consists of alternating between output voltage states by half a period to produce an AC square wave. This method is known as the square wave modulation [14]. Square wave modulation contains odd harmonics of the fundamental frequency, thus requiring extensive filtering to isolate the fundamental frequency. The generated harmonics reduce efficiency and power quality; thus, making the control strategy undesirable to high power applications. These deficiencies may be prevented by implementing selective harmonic elimination (SHE) or bipolar sinusoidal pulse width modulation (SPWM) method [14]. Each method possesses unique characteristics and are highlighted to identify suitable applications.

The SHE method demonstrates improved efficiency and power quality relative to the square wave inverter [14]. SHE requires mathematically generating timing between the two output voltage states to effectively null harmonics of the fundamental frequency. The 
mathematical generation of switching states is achieved using Fourier analysis [14]. The output waveform and frequency spectrum of the SHE method is abstracted from [14], where the third and fifth order harmonics are eliminated. This control strategy is worthy of applications where an unwanted harmonic is significant or may cause instability, such as the triplen harmonics associated with power systems. Unfortunately, the harmonics produced by SHE are significantly close to the fundamental frequency, thus complicating the task of filtering. Even though SHE is capable of eliminating harmonics, the applications of the method are limited and higher efficiencies are achieved using bipolar sinusoidal pulse width modulation (SPWM).

SPWM is the most common control method for inverters and the bipolar SPWM method is the simplest to implement. Implementation of bipolar SPWM is realized by comparing a sinusoidal wave of a desired low frequency to a higher frequency triangle wave [14]. The resulting control signals are used to drive the switches in the half-bridge topology. The benefit of bipolar SPWM is the separation of the sine wave fundamental frequency from the high switching frequency; thus, reducing the filtering requirement and increasing the quality and efficiency of the AC output. In addition, the amplitude of the output voltage is controllable by selecting the ratio between the triangular and sinusoidal waveforms [14]. The drawback of the bipolar SPWM method is the increased need for controls, thus increasing the cost of the system relative to the square wave method. Additionally, the resulting ac output waveform contains significant high frequency pulses that introduce additional switching losses in the inverter as well as Electromagnetic Interference (EMI) noise.

\subsubsection{Strengths and Weaknesses}

The simplicity of the half-bridge topology results in two main advantages. Low system costs are achieved, since minimal components are required for half bridge operation. In addition, simple control strategies further reduce system costs. Although the half-bridge 
topology offers a low cost system, the performance of the topology is severely impacted relative to other topologies, which are discussed in the following sections. Additional advantage includes effective use of its transformer due to the four-quadrant flux excursion which implies smaller sized transformer compared to other isolated topologies which utilizes one quadrant of their transformer's B-H curve.

The disadvantages of this topology are mainly due to minimized output voltage states and the requirement of a split supply. Since only two output voltage states are achievable, limited control strategies may be implemented in the half-bridge. A split supply is achieved using two well matched capacitors [14]; however, low frequency switching applications cause voltage imbalances in this split supply method [16]. In addition, voltage imbalances also occur as a result of leakage currents in the capacitors [15]. Balancing resistors are used to achieve equal voltages, though this introduces losses to the system. The literature, [15] and [16], demonstrates methods to achieve voltage balance. [16] proposes the use of a constant frequency control to achieve voltage balance; though, this comes at the cost of reduced efficiencies at lighter loads.

\subsection{Full-Bridge Inverter}

The full bridge inverter, also known as the H-bridge, incorporates a total of four switches and is shown in Figure 2-3. The full bridge may achieve a maximum of three states and the requirement for a split supply is eliminated. 


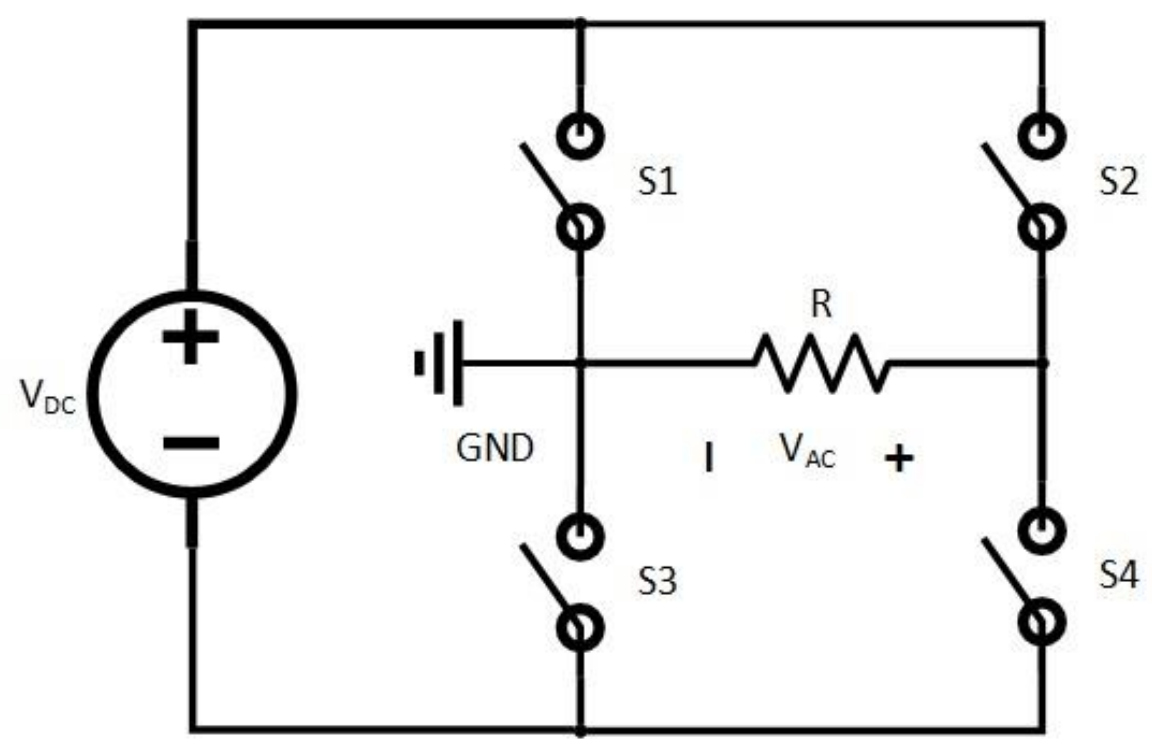

Figure 2-3: Full bridge inverter

When SW1 and SW4 are on, SW2 and SW3 are off, resulting in a positive voltage across the load. When SW2 and SW3 are on, SW1 and SW4 are off, resulting in a negative voltage across the load. A zero voltage state is achieved when both SW1 and SW2 are on, or when both SW3 and SW4 are on. SW1 and SW3 form a leg of the inverter, and the two switches should never be on at the same time to prevent shorting the DC supply. Likewise, SW2 and SW4 form another leg and should never be on at the same time. 
Table 2-2: Full bridge inverter output voltage states

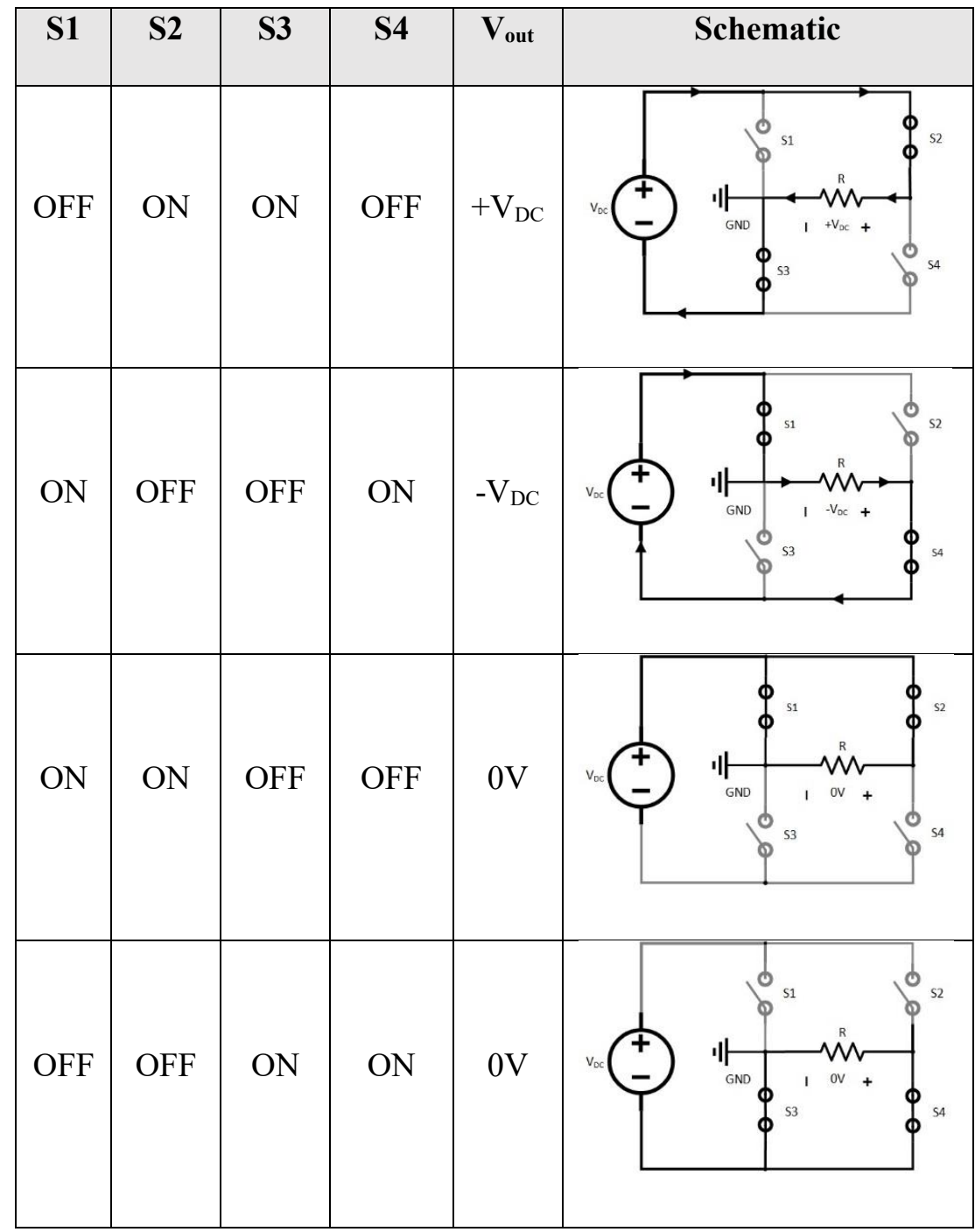

\subsubsection{Controls}

The full bridge topology is capable of employing the control strategies proposed for the half-bridge inverter; however, these strategies do not make use of the $0 \mathrm{~V}$ state. Thus, conventional control strategies for the half-bridge topology offer limited performance. A unipolar SPWM control method is employed to effectively utilize all states of the full bridge topology, thus increasing performance [14].

The unipolar SPWM method overcomes the deficiencies of the bipolar SPWM method by utilizing more switching states. In addition to the aforementioned DC rail 
voltages, the unipolar SPWM method utilizes the zero voltage state of the full bridge topology. Controls are realized by comparing two sinusoidal reference signals of a fundamentally low frequency to a high frequency triangular wave [14]. The two sinusoidal reference signals have the same frequency, but are out of phase by $180^{\circ}$.

Similar to bipolar SPWM, unipolar SPWM utilizes a series of pulses with modulated duty cycles; however, the zero voltage state is used to effectively suppress transient switching voltages. This reduces the filtering requirement of the output filtering stage, since harmonics of the switching frequency are diminished. The disadvantage of the unipolar method is the increased need for controls, since a secondary reference signal is required [14]. The increase in controls corresponds to an increase in system price; thus, unipolar SPWM method is commonly used in high performance systems where efficiency, power quality, and cost must be optimized.

\subsubsection{Strengths and Weaknesses}

The benefit of the full bridge inverter lies within the additional $0 \mathrm{~V}$ state. Utilizing this state diminishes switching voltage transients, which in turn reduces output voltage harmonic content. The reduced harmonic content of the output voltage eases the filtering requirement of the output filter; thus reducing the size and cost of the output filter. Additionally, the full bridge topology is susceptible to supplementary switching techniques relative to the half-bridge, which increase system performance flexibility. For these reasons, the full bridge topology is implemented in systems where power quality and efficiency must be balanced with system cost.

Disadvantages of the full-bridge inverter include the use of more switches. The increased number of switches results in greater conduction losses. Additionally, switching losses increase as a result of the increase in the number of switches. Furthermore, controls are 
required to drive the four switches, which grows control system complexity and requires peripheral functions to accurately control switching.

\subsection{Multi-Level Inverter}

The multi-level inverter combines multiple discrete voltage levels to form an AC output. The topology of a three level inverter is demonstrated in Figure 2-4. Though this figure only demonstrates the topology for a three level inverter, it is important to remember that this concept may be extracted to multiple levels by stacking multiple switches to increase the number of allowed states. Note that increasing the number of states directly increases the number of split supplies required; thus complicating the topology, since voltage balance for each source would be required.

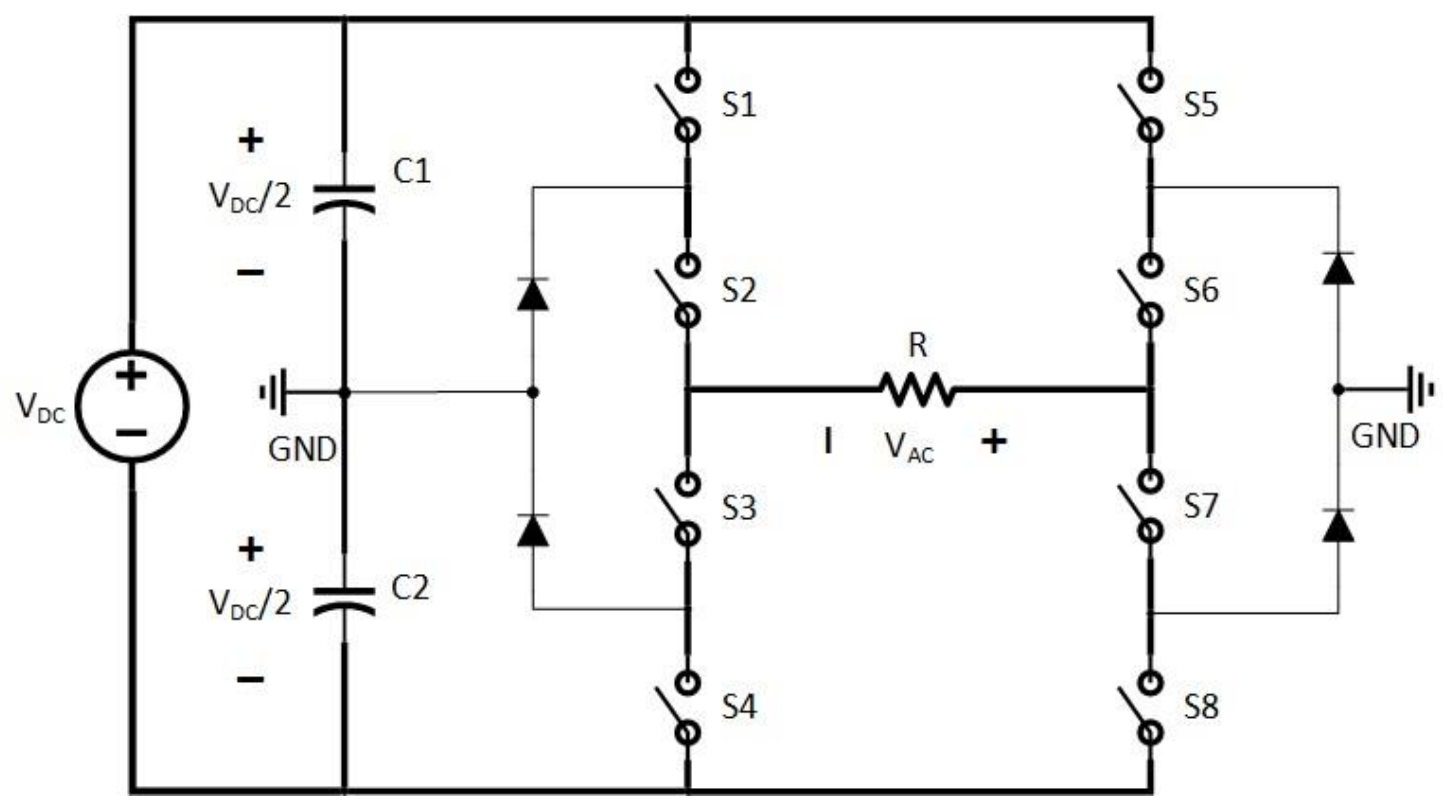

Figure 2-4: Three-level inverter

The operation of the multi-level inverter is achieved by alternating states of the inverter.

To define the practical use of the multi-level inverter, the switching states of a 3-level inverter are demonstrated in Table 2-3. In a 3-level inverter a total of 5 states are achievable. The practical voltage states are as follows: +VDC, $+0.5 \mathrm{VDC}, 0 \mathrm{~V},-0.5 \mathrm{VDC}$, and -VDC. 
Table 2-3: Three level multi-level inverter output voltage states

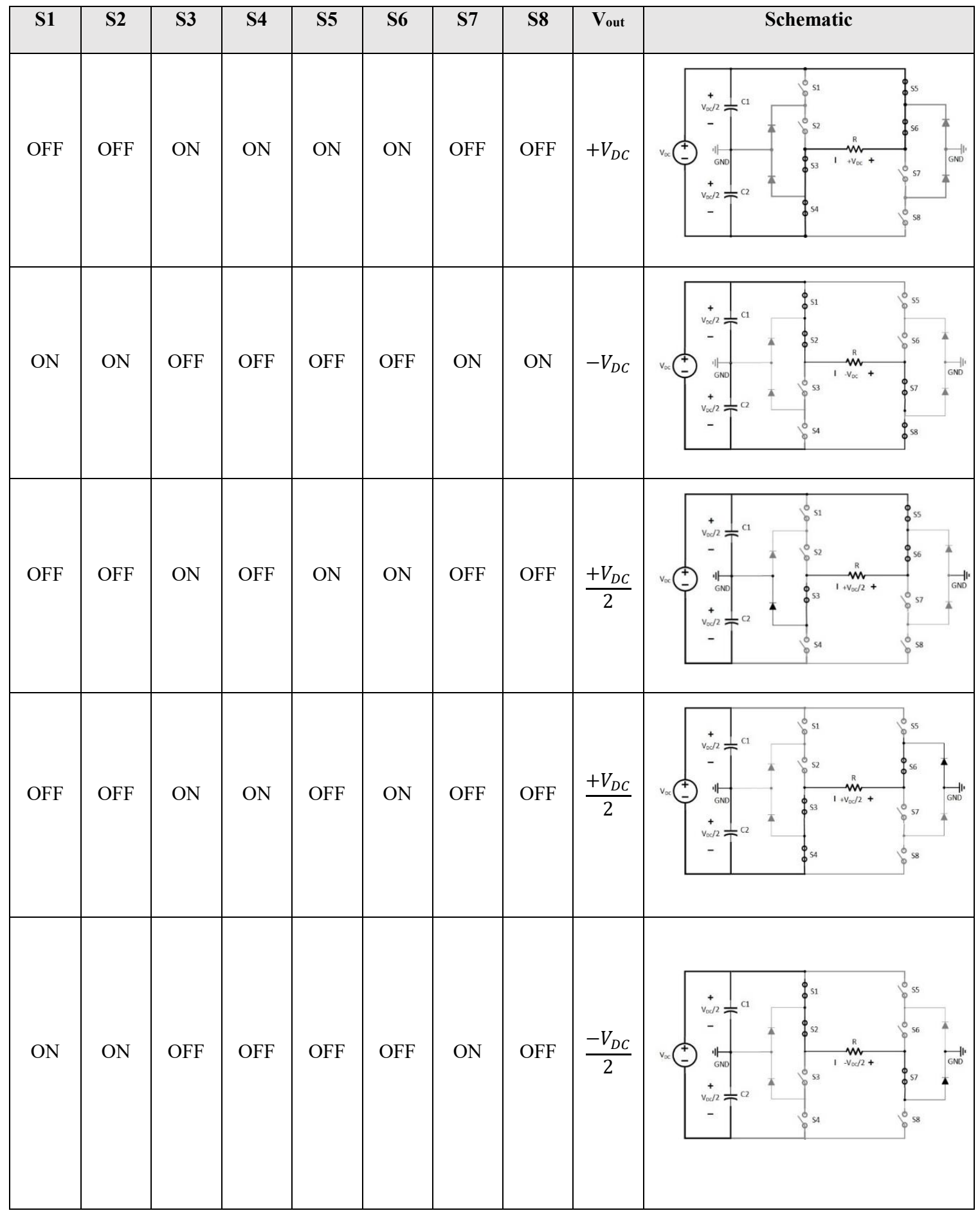




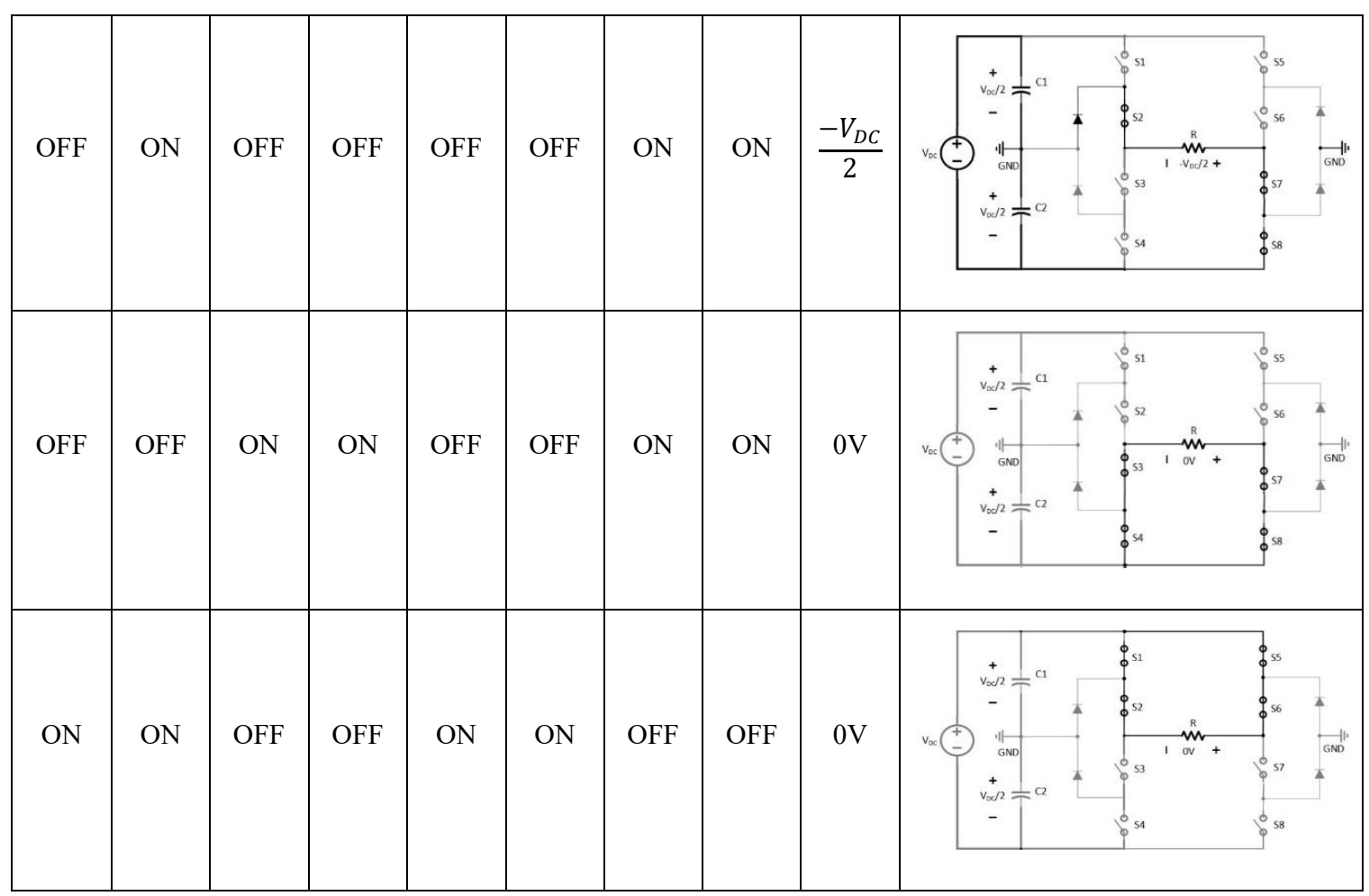

\subsubsection{Controls}

Control of the multi-level inverter is implemented using a multi-level unipolar SPWM method. The maximum switch stress for the multi-level inverter topology is $\mathrm{V}_{\mathrm{DC}} / 2$, which is evident by the switching states shown in Table 2-3: Three level multi-level inverter output voltage states. This makes the multi-level inverter suitable for high voltage applications, since switching losses are minimized, due to the reduced voltage stress. The multi-level unipolar SPWM method is the most common for multi-level inverter topologies [17]. Although many multi-level SPWM strategies exist, all methods compare a triangular waveform reference to a sinusoidal reference to generate control signals [17]. Strategies shown in [17] differ by altering the phase and offset of the reference signals. The control signals required for multi-level inverters increase as the number of levels increases; however, these systems exhibit high efficiencies and flexible performance at high power demands [18]. 


\subsubsection{Strengths and Weaknesses}

Performance of the multi-level inverter is adjustable depending on the application. As discussed, the topology of the inverter is adjustable to accommodate the amount of necessary states for a given design. This suits high voltage applications well, since the voltage stress induced on switches is inversely proportional to the number of states. This means that for a high voltage application, many switches may be stacked to reduce switch voltage stress with minimal impact on performance [18]. In addition, the diminished switching transients reduce the harmonic content of the output, thus reducing the filtering requirement of the output filter. This leads to a reduced cost and size of the output filtering stage.

Though these systems exhibit excellent performance, the caveat is an increase in controls and components. Greater system performance is achieved by increasing the number of allotted switching states. In turn, this causes an increase in the size and cost of the control system. Efforts to reduce components are demonstrated in [19]; however, isolated DC

supplies are required for each level of the inverter, which increases cost of the input stage of the inverter system. Moreover, multi-level inverters require split supplies to achieve switch voltage stress reduction; thus, the same issues regarding voltage balancing presented in [15] and [16] plague the multi-level inverter. Though multi-level inverter is complex and expensive when compared to full and half-bridge topologies, these systems are an excellent fit for high power, high voltage systems that require performance flexibility.

\subsection{Resonant Inverter}

The resonant inverter is unlike previously discussed topologies, as the output voltage waveform does not contain discrete voltage levels. Instead, a step input is applied to a second order system to induce an underdamped response, resulting in a sinusoidal voltage output. A series loaded half-bridge resonant inverter is shown in Figure 2-5. Although variations of the 
resonant inverter topology exist [20], the focus of this thesis is the series loaded resonant inverter, due to the potential for high efficiencies as switching losses are eliminated.

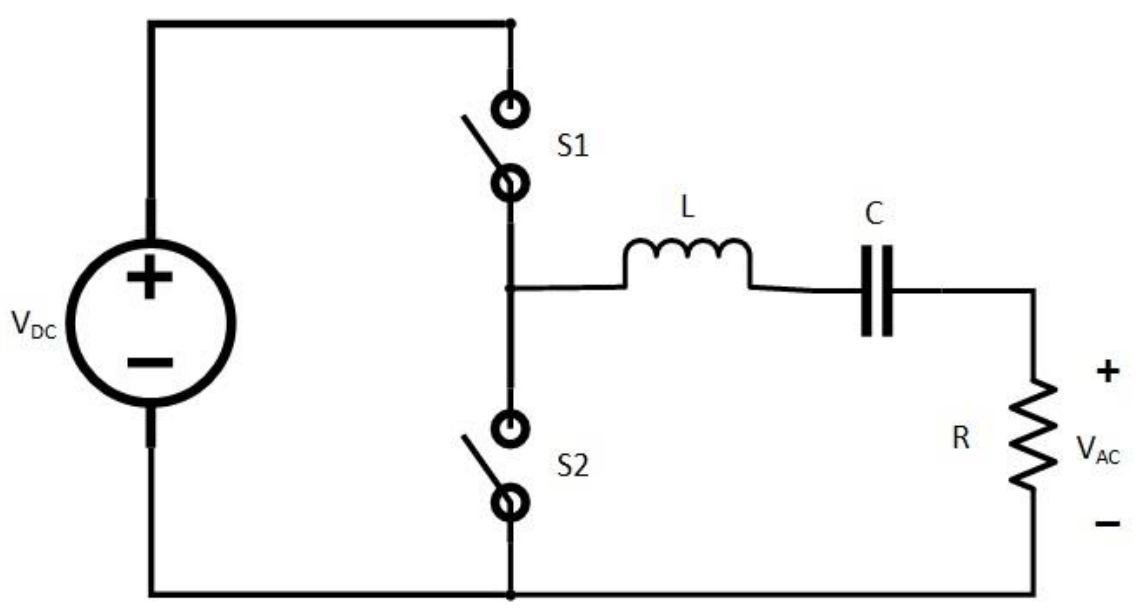

Figure 2-5: Series loaded resonant inverter

Similar to the half-bridge topology, the resonant inverter is only capable of two states for the output voltage; however, a NPC configuration is not necessary, since the output capacitor eliminates the DC component of the output. To achieve these states, a step response is introduced to a second order system composed of inductor, capacitor, and load resistor. The resonant frequency of the network is designed to be equivalent to the desired output frequency. When the inverter switches between states, the resonant network causes an oscillation at the output of the inverter. The output states of the series resonant inverter are summarized in Table 2-4. 
Table 2-4: Series loaded resonant inverter output voltage states

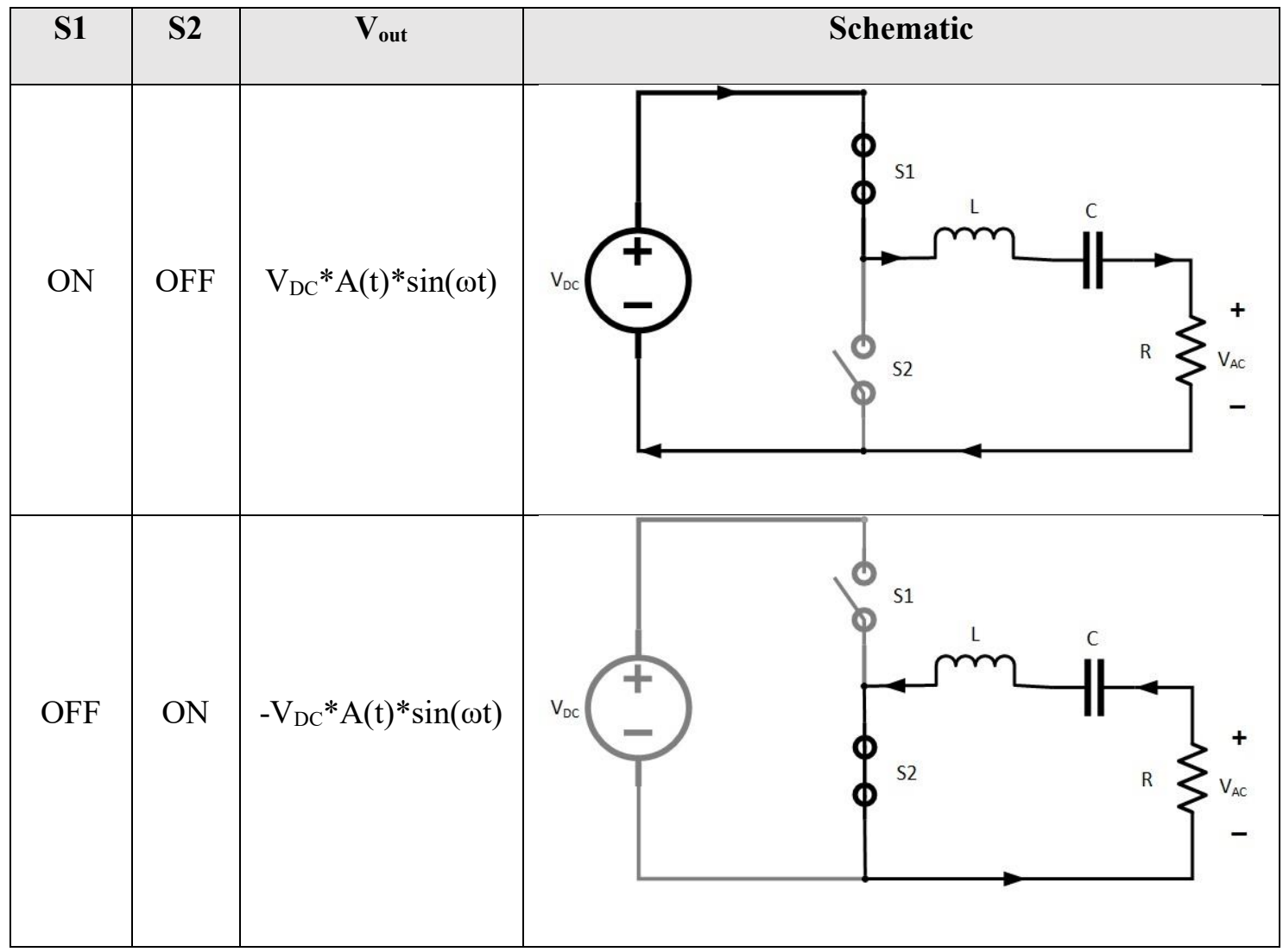

Note that the output of SLR inverter is not exactly sinusoidal. An additional term, $A(t)$, is used to describe the time domain output of the SLR inverter. This term attenuates the sinusoid and is a function of time. This term is further discussed in the following chapter. The literature demonstrates a great focus on resonant inverters for induction cooktops and ballasts for lighting [21][22]. These applications require high frequency operation; however, this thesis aims to model the SLR inverter in low frequency applications to identify suitable applications.

\subsubsection{Controls}

Control of the series loaded resonant inverter is realized by square wave modulation.

This is the simplest control strategy for inverters. Ideally, the frequency spectrum will only contain a fundamental frequency equivalent to the resonant frequency of the LC network; 
however, switching transients induce harmonics causing distortion of the output. The effects of harmonic distortion are characterized in this thesis. It is important to note that significant path losses contribute to the overall distortion of the output. The influence of path loss is investigated to determine suitable applications for the low frequency SLR inverter.

The self-oscillating nature of the resonant inverter allows for control without the need of a controller. This is evident in [23] where an IC-less control system for self-resonating structures is demonstrated. Energy is coupled from the main inductor to drive the half-bridge switches. This is a good solution for high frequency applications; however, low-frequencies require significant windings to drive switches. Nevertheless, a solution may be constructed using the information from [23] to develop a self-oscillating control suited for low frequencies.

\subsubsection{Strengths and Weaknesses}

The simplicity of the resonant inverter is a major benefit, since only 2 switches are required. The simple two switch topology minimizes conduction losses and system size. In addition, the resonant nature of the inverter results in zero-current switching (ZCS), thus eliminating switching losses allowing for high voltage operation. Control of the resonant inverter is achieved using a simple square wave technique, which results in reduced size and cost of the control system. These benefits allow high voltage operation leading to increased efficiencies; however, frequency stability limits applications for the SLR inverter.

Although resonant inverter has the potential of being a high performance inverter, frequency stability is highly load dependent. Output voltage and frequency are both dependent on load and resistive parasitic in the series LC path. These parameters are difficult to control since a change in the load will effectively change the performance of the inverter. Although these flaws are well known to limit resonant inverter operation, little research has 
been conducted on the characterization of these limitations. This thesis characterizes the series loaded resonant topology to further understand the limitations and practicality of use.

\subsubsection{Advancements}

Advancements in the field of power electronics suggest an electronically tunable inductor [23][25][26]. Electro-tunable inductor technology has existed in the past for RF applications [23]; however, recent advancements demonstrate larger tuning ranges and increased quality factors all within lower operational frequencies [25][26]. The magneto-

electric inductor is reported to have a tuning range of up to $370 \%\left(\mathrm{~L}_{\max } / \mathrm{L}_{\min }=370 \%\right)$ [25]. Additionally, quality factors up to 24 have been reported, though dependent on control voltage. These advancements may be a feasible solution to the frequency stability concerning resonant inverters. The frequency range required to maintain stability is demonstrated in this thesis. In addition, a design methodology is presented to determine optimum selection of inductance for the SLR inverter based on load and desired frequency of operation. 


\section{Chapter 3: Resonant Inverter Characterization}

A model is developed to determine the optimal selection of inductance and capacitance for the series loaded resonant inverter. A transfer function method is used to develop the SLR inverter model in the frequency domain, followed by a Laplace transformation to determine a solution in the time domain. Similar models exist in the literature, [27]; however, this method uses a phase plane trajectory to analyze the model. The phase plane trajectory model lends well for analyzing stability, though fails to identify RMS output voltage and efficiency. The proposed model analyzes RMS output voltage/current, efficiency, and determines component sizing of inductor, capacitor, and switches. In addition, a design strategy to select optimum inductance and capacitance is presented. The optimal selection of inductance and capacitance is based on a well characterized load and desired frequency of operation.

\subsection{Series Loaded Resonant Inverter Model}

The SLR model is first developed by applying the voltage divider equation to the circuit shown in Figure 3-1, which demonstrates the frequency domain representation of the RLC network at the SLR inverter output. The resulting equation is shown below.

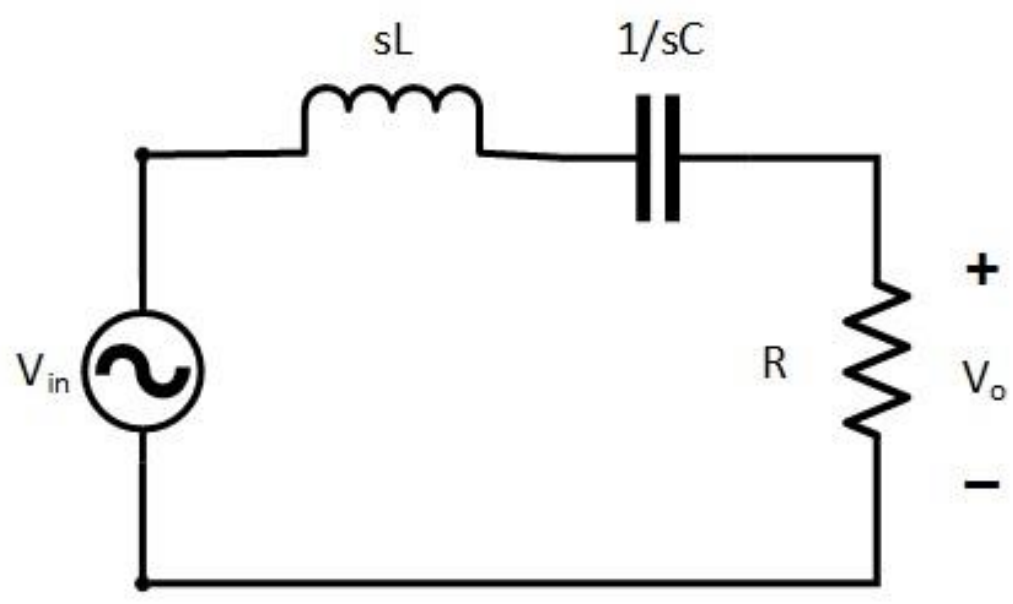

Figure 3-1: Frequency domain representation of output RLC network 


$$
V_{o}=V_{\text {in }} \frac{R_{L}}{R_{L}+R_{s}+R_{e s r}+R_{D S(o n)}+s L+\frac{1}{s C}}
$$

Equation (3-1) accounts for the inductor winding resistance, $R_{S}$, capacitor equivalent series resistance, $R_{e s r}$, and MOSFET on resistance, $R_{D S(o n)}$. The summation of these resistances are referred to as the total series resistance, $R_{\text {total }}$, shown below.

$$
R_{\text {total }}=R_{L}+R_{S}+R_{e s r}+R_{D S(o n)}
$$

A step input is introduced to the transfer function to account for the switching between states of the half-bridge inverter. The Laplacian step input is given below.

$$
V_{\text {in }}=\frac{V_{D C}}{S}
$$

Combining equations (3-1), (3-2), and (3-3) results in,

$$
V_{o}=\frac{V_{D C}}{s} * \frac{R_{L}}{R_{\text {total }}+s L+\frac{1}{s C}}=\frac{V_{D C} R_{L}}{L} * \frac{1}{s^{2}+s \frac{R_{\text {total }}}{L}+\frac{1}{L C}}
$$

Completing the square for the denominator,

$$
V_{o}=\frac{V_{D C} R_{L}}{L} * \frac{1}{\left(s+\frac{R_{\text {total }}}{2 L}\right)^{2}+\frac{1}{L C}-\left(\frac{R_{\text {total }}}{2 L}\right)^{2}}
$$

Fitting the function for an exponentially decaying sine wave, which is a known Laplace transformation,

$$
V_{o}=\frac{V_{D C} R_{L}}{L} * \frac{1}{\sqrt{\frac{1}{L C}-\left(\frac{R_{\text {total }}}{2 L}\right)^{2}}} * \frac{\sqrt{\frac{1}{L C}-\left(\frac{R_{\text {total }}}{2 L}\right)^{2}}}{\left(s+\frac{R_{\text {total }}}{2 L}\right)^{2}+\frac{1}{L C}-\left(\frac{R_{\text {total }}}{2 L}\right)^{2}}
$$


Taking the inverse Laplace Transform,

$$
\begin{aligned}
& v_{o}(t)=\left[\frac{V_{D C} R_{L}}{L \sqrt{\frac{1}{L C}-\left(\frac{R_{\text {total }}}{2 L}\right)^{2}}}\right] *\left[e^{-\left(\frac{R_{\text {total }}}{2 L}\right) t}\right] *\left[\sin \left(\sqrt{\frac{1}{L C}-\left(\frac{R_{\text {total }}}{2 L}\right)^{2}}\right) t\right] * u(t) \\
& =\left[\frac{V_{D C} R_{L}}{\sqrt{\frac{L}{C}-\left(\frac{R_{\text {total }}}{2}\right)^{2}}}\right] *\left[e^{-\left(\frac{R_{\text {total }}}{2 L}\right) t}\right] *\left[\sin \left(\sqrt{\frac{1}{L C}-\left(\frac{R_{\text {total }}}{2 L}\right)^{2}}\right) t\right] * u(t)
\end{aligned}
$$

The above equation may be reduced by assuming the parameters $R_{L}, R_{\text {total }}, L$, and $C$ are constant. Constants are used to simplify the derived equation and their significance is defined below.

Note that the sine wave is attenuating by a constant factor, $\alpha$. This is known as the attenuation factor and determines the rate at which the sine wave attenuates and is defined below.

$$
\alpha=\frac{R_{\text {total }}}{2 L}
$$

The resonant frequency is defined in equation (3-5). The resonant frequency is the ideal frequency of resonance for the case where resistance is absent in the series path. In practice, series resistances are always present and thus affect the frequency of the resonating output.

$$
\omega_{o}=\sqrt{\frac{1}{L C}}
$$

The actual frequency of the sine wave resonance is known as the damped frequency, $\omega_{d}$. Equation (3-6) demonstrates the damped frequency as a function of the total series resistance. Note that as the resistance of the series path increases the damped frequency decreases. This brings about an important concern with resonant inverters, since a load change will inherently cause a deviation in frequency. 


$$
\omega_{d}=\sqrt{\frac{1}{L C}-\left(\frac{R_{\text {total }}}{2 L}\right)^{2}}=\sqrt{\omega_{o}^{2}-\alpha^{2}}
$$

The characteristic impedance is defined by equation (3-7). The characteristic impedance is known as the natural resistance of the second order system, when series path resistances are absent.

$$
Z_{o}=\sqrt{\frac{L}{C}}
$$

The total effective impedance is defined in equation (3-8). In practice, series path resistances are always present, thus diminishing the effective impedance of the second order system.

$$
Z_{\text {eff }}=\sqrt{\frac{L}{C}-\left(\frac{R_{t o t a l}}{2}\right)^{2}}=\sqrt{Z_{o}^{2}-(a L)^{2}}
$$

Equations (3-4) to (3-8) simplify the output voltage to the equation shown below.

$$
v_{o}(t)=\left[\frac{V_{D C} R_{L}}{Z_{e f f}}\right] *\left[e^{-\alpha t}\right] *\left[\sin \omega_{d} t\right] * u(t)
$$

Equation (3-9) is the result of a step input to the second order system formed by series R, L, C components. This step input occurs at the SLR inverter switch node. A positive step input occurs when the low side switch turns off and the high side switch turns on. Similarly, a negative step input occurs when the high side switch turns off and the low side switch turns on. For a negative step input, the output voltage described by equation (3-9) is negative. In both cases, the circuit parameters are equivalent, except for the polarity of the step input. The SLR inverter transitions between positive and negative voltage states every half-period established by the damped resonant frequency $\omega_{\mathrm{d}}$. Initial conditions are ignored for every switching transition to 
simplify the mathematical model of the SLR inverter. The mathematical model of the SLR inverter is provided below.

$$
v_{o}(t)=A *\left[\frac{V_{D C} R_{L}}{Z_{\text {eff }}}\right] *\left[e^{-\alpha t}\right] *\left[\sin \omega_{d} t\right]\left\{\begin{array}{cl}
A=1, & 0 \leq \omega_{d} t<\pi \\
A=-1, & \pi \geq \omega_{d} t<2 \pi
\end{array}\right.
$$

The voltage was derived for a purely resistive load; thus, Ohm's law is used to find the corresponding output current.

$$
i_{o}(t)=\frac{v_{o}(t)}{R_{L}}=A *\left[\frac{V_{D C}}{Z_{e f f}}\right] *\left[e^{-\alpha t}\right] *\left[\sin \omega_{d} t\right]\left\{\begin{array}{cl}
A=1, & 0 \leq \omega_{d} t<\pi \\
A=-1, & \pi \geq \omega_{d} t<2 \pi
\end{array}\right.
$$

Equations (3-4) to (3-11) define the output voltage and current of the SLR inverter. It is important to note that these equations disregard initial conditions. Impact of initial conditions are further discussed in the analysis section of this thesis. An analysis of the derived model reveals pertinent system parameters described below.

\subsection{RMS Output Voltage/Current}

Solving for the root mean square (RMS) of the output voltage where $v_{o}(t)$ is given in equation (3-10) and the RMS is defined as,

$$
\widetilde{V_{o}}=\sqrt{\frac{1}{T_{d}} \int_{-T_{d} / 2}^{T_{d} / 2} v_{o}^{2}(t) d t}
$$

where $T_{d}$ is the period of the damped oscillation,

$$
T_{d}=\frac{2 \pi}{\omega_{d}}=\frac{2 \pi}{\sqrt{\frac{1}{L C}-\left(\frac{R_{\text {total }}}{2 L}\right)^{2}}}
$$

and, 


$$
v_{o}^{2}(t)=\left[\left(\frac{V_{D C} R_{L}}{Z_{e f f}}\right)\left(e^{-\alpha t}\right)\left(\sin \omega_{d} t\right)\right]^{2}=\left(\frac{V_{D C} R_{L}}{Z_{e f f}}\right)^{2}\left(e^{-2 \alpha t}\right)\left(\sin ^{2} \omega_{d} t\right)
$$

By the half angle identity,

$$
v_{o}^{2}(t)=\frac{1}{2}\left(\frac{V_{D C} R_{L}}{Z_{e f f}}\right)^{2}\left(e^{-2 \alpha t}\right)\left(1-\cos 2 \omega_{d} t\right)
$$

$v_{o}(t)^{2}$ repeats every half cycle. The integral is performed for a half period using symmetry,

$$
\begin{gathered}
\widetilde{V}_{o}=\sqrt{\frac{1}{T_{d}} \int_{-T_{d} / 2}^{T_{d} / 2} v_{o}^{2}(t) d t}=\sqrt{\frac{2}{T_{d}} \int_{0}^{T_{d} / 2} v_{o}^{2}(t) d t} \\
=\sqrt{\frac{2}{T_{d}} \int_{0}^{T_{d} / 2} \frac{1}{2}\left(\frac{V_{D C} R_{L}}{Z_{e f f}}\right)^{2}\left(e^{-2 \alpha t}\right)\left(1-\cos 2 \omega_{d} t\right) d t} \\
\widetilde{V}_{o}=\sqrt{\frac{1}{T_{d}}\left(\frac{V_{D C} R_{L}}{Z_{e f f}}\right)^{2} \int_{0}^{T_{d} / 2}\left(e^{-2 \alpha t}\right)\left(1-\cos 2 \omega_{d} t\right) d t}
\end{gathered}
$$

The integral of equation (3-13) is solved using a table of integrals [28]. The solution is shown below, 


$$
\begin{aligned}
& \int_{0}^{\frac{T_{d}}{2}}\left(e^{-2 \alpha t}\right)\left(1-\cos 2 \omega_{d} t\right) d t=\int_{0}^{\frac{T_{d}}{2}}\left(e^{-2 \alpha t}\right) d t-\int_{0}^{\frac{T_{d}}{2}}\left(e^{-2 \alpha t}\right)\left(\cos 2 \omega_{d} t\right) d t \\
& =\frac{e^{-2 \alpha t}}{-2 \alpha}-\left.\frac{e^{-2 \alpha t}}{\left(2 \omega_{d}\right)^{2}+(-2 \alpha)^{2}}\left(2 \omega_{d} \sin 2 \omega_{d} t-2 \alpha \cos 2 \omega_{d} t\right)\right|_{0} ^{\frac{T_{d}}{2}} \\
& =\left.e^{-2 \alpha t}\left(\frac{1}{-2 \alpha}-\frac{1}{\left(2 \omega_{d}\right)^{2}+(-2 \alpha)^{2}}\left(2 \omega_{d} \sin 2 \omega_{d} t-2 \alpha \cos 2 \omega_{d} t\right)\right)\right|_{0} ^{\frac{T_{d}}{2}} \\
& =e^{-2 \alpha \frac{T_{d}}{2}}\left(\frac{1}{-2 \alpha}-\frac{1}{\left(2 \omega_{d}\right)^{2}+(2 \alpha)^{2}}\left(2 \omega_{d} \sin 2 \omega_{d} \frac{T_{d}}{2}-2 \alpha \cos 2 \omega_{d} \frac{T_{d}}{2}\right)\right) \\
& -e^{0}\left(\frac{1}{-2 \alpha}-\frac{1}{\left(2 \omega_{d}\right)^{2}+(2 \alpha)^{2}}\left(2 \omega_{d} \sin 0-2 \alpha \cos 0\right)\right) \\
& =e^{-\alpha T_{d}}\left(\frac{1}{-2 \alpha}+\frac{2 \alpha}{\left(2 \omega_{d}\right)^{2}+(2 \alpha)^{2}}\right)-\left(\frac{1}{-2 \alpha}+\frac{2 \alpha}{\left(2 \omega_{d}\right)^{2}+(2 \alpha)^{2}}\right) \\
& =\left(\frac{1}{-2 \alpha}+\frac{2 \alpha}{\left(2 \omega_{d}\right)^{2}+(2 \alpha)^{2}}\right)\left(e^{-\alpha T_{d}}-1\right) \\
& =\frac{2 \alpha}{2 \alpha}\left[\left(\frac{1}{-2 \alpha}+\frac{2 \alpha}{\left(2 \omega_{d}\right)^{2}+(2 \alpha)^{2}}\right)\left(e^{-\alpha T_{d}}-1\right)\right] \\
& =\frac{1}{2 \alpha}\left(-1+\frac{(2 \alpha)^{2}}{\left(2 \omega_{d}\right)^{2}+(2 \alpha)^{2}}\right)\left(e^{-\alpha T_{d}}-1\right) \\
& =\frac{1}{2 \alpha}\left(-1+\frac{\alpha^{2}}{\omega_{d}^{2}+\alpha^{2}}\right)\left(e^{-\alpha T_{d}}-1\right)
\end{aligned}
$$

Where $\omega_{o}^{2}=\omega_{d}^{2}+\alpha^{2}$ by equation (3-6),

$$
\begin{aligned}
\int_{0}^{T_{d} / 2}\left(e^{-2 \alpha t}\right)(1 & \left.-\cos 2 \omega_{d} t\right) d t=\frac{1}{2 \alpha}\left(-1+\frac{\alpha^{2}}{\omega_{o}^{2}}\right)\left(e^{-\alpha T_{d}}-1\right) \\
& =\frac{1}{2 \alpha}\left(-\frac{\omega_{o}^{2}}{\omega_{o}^{2}}+\frac{\alpha^{2}}{\omega_{o}^{2}}\right)\left(e^{-\alpha T_{d}}-1\right)=\frac{1}{2 \alpha}\left(\frac{-1}{-1}\right)\left(\frac{\alpha^{2}-\omega_{o}^{2}}{\omega_{o}^{2}}\right)\left(e^{-\alpha T_{d}}-1\right) \\
& =\frac{1}{2 \alpha}\left(\frac{\omega_{o}^{2}-\alpha^{2}}{\omega_{o}^{2}}\right)\left(1-e^{-\alpha T_{d}}\right)
\end{aligned}
$$


Once again, substituting equation (3-6) reduces the integral to,

$$
\int_{0}^{T_{d} / 2}\left(e^{-2 \alpha t}\right)\left(1-\cos 2 \omega_{d} t\right) d t=\frac{\omega_{d}^{2}}{2 \alpha \omega_{o}^{2}}\left(1-e^{-\alpha T_{d}}\right)
$$

Finally, combining equations (3-13) and (3-14) the output RMS voltage is as follows:

$$
\begin{gathered}
\widetilde{V}_{o}=\sqrt{\frac{1}{T_{d}}\left(\frac{V_{D C} R_{L}}{Z_{e f f}}\right)^{2}\left[\int_{0}^{\frac{T_{d}}{2}}\left(e^{-2 \alpha t}\right)\left(1-\cos 2 \omega_{d} t\right) d t\right]}=\sqrt{\frac{1}{T_{d}}\left(\frac{V_{D C} R_{L}}{Z_{e f f}}\right)^{2}\left[\frac{\omega_{d}^{2}}{2 \alpha \omega_{o}^{2}}\left(1-e^{-\alpha T_{d}}\right)\right]} \\
=\frac{V_{D C} R_{L} \omega_{d}}{Z_{e f f} \omega_{o}} \sqrt{\frac{1-e^{-\alpha T_{d}}}{2 \alpha T_{d}}}
\end{gathered}
$$

Plugging in equations (3-5), (3-6), and (3-8).

$$
\begin{gathered}
\widetilde{V}_{o}=\frac{V_{D C} R_{L}\left(\sqrt{\frac{1}{L C}-\left(\frac{R_{\text {total }}}{2 L}\right)^{2}}\right)}{\left(\sqrt{\frac{L}{C}-\left(\frac{R_{\text {total }}}{2}\right)^{2}}\right)\left(\sqrt{\frac{1}{L C}}\right) \sqrt{\frac{1-e^{-\alpha T_{d}}}{2 \alpha T_{d}}}} \\
=V_{D C} R_{L} \sqrt{\frac{\frac{1}{L C}-\left(\frac{R_{\text {total }}}{2 L}\right)^{2}}{\left(\frac{L}{C}-\left(\frac{R_{\text {total }}}{2}\right)^{2}\right)\left(\frac{1}{L C}\right)}\left(\frac{L^{2}}{L^{2}}\right) \sqrt{\frac{1-e^{-\alpha T_{d}}}{2 \alpha T_{d}}}} \\
=V_{D C} R_{L} \sqrt{\frac{\frac{L}{C}-\left(\frac{R_{\text {total }}}{2}\right)^{2}}{\left(\frac{L}{C}-\left(\frac{\left.\left.R_{\text {total }}\right)^{2}\right)\left(\frac{L}{C}\right)}{2}\right.\right.} \sqrt{\frac{1-e^{-\alpha T_{d}}}{2 \alpha T_{d}}}=V_{D C} R_{L} \frac{1}{\sqrt{\frac{L}{C}}} \sqrt{\frac{1-e^{-\alpha T_{d}}}{2 \alpha T_{d}}}}
\end{gathered}
$$

Substituting equation (3-7) yields, 


$$
\widetilde{V}_{o}=\frac{V_{D C} R_{L}}{Z_{o}} \sqrt{\frac{1-e^{-\alpha T_{d}}}{2 \alpha T_{d}}}
$$

Since the RMS output voltage was derived for a purely resistive load, the RMS output current is determined by Ohm's law.

$$
\widetilde{I_{o}}=\frac{\widetilde{V_{o}}}{R_{L}}=\frac{1}{R_{L}} \frac{V_{D C} R_{L}}{Z_{o}} \sqrt{\frac{1-e^{-\alpha T_{d}}}{2 \alpha T_{d}}}
$$

Thus,

$$
\widetilde{I}_{o}=\frac{V_{D C}}{Z_{o}} \sqrt{\frac{1-e^{-\alpha T_{d}}}{2 \alpha T_{d}}}
$$

\subsection{Output Power}

The output power delivered to the load is derived using the power law. Additionally, equations (3-16) and (3-15) are used in conjunction to solve for the output power. The derivation is shown below.

$$
\begin{gathered}
P_{o}=\widetilde{V}_{o} \widetilde{I}_{o}=\left(\frac{V_{D C} R_{L}}{Z_{o}} \sqrt{\frac{1-e^{-\alpha T_{d}}}{2 \alpha T_{d}}}\right)\left(\frac{V_{D C}}{Z_{o}} \sqrt{\frac{1-e^{-\alpha T_{d}}}{2 \alpha T_{d}}}\right)=\frac{V_{D C}{ }^{2} R_{L}}{Z_{o}{ }^{2}}\left(\frac{1-e^{-\alpha T_{d}}}{2 \alpha T_{d}}\right) \\
P_{o}=\frac{V_{D C}{ }^{2} R_{L}}{Z_{o}{ }^{2}}\left(\frac{1-e^{-\alpha T_{d}}}{2 \alpha T_{d}}\right)
\end{gathered}
$$

\subsection{Optimum DC to AC RMS Gain}

Determining optimum inductance to obtain maximum RMS output voltage for a given total path resistance and desired frequency of operation. To determine the optimum inductance, the derivative of the output RMS voltage, $\widetilde{V_{o}}$, is taken with respect to inductance. The result is set 
to zero and a solution is established for the maximum output RMS voltage for a given inductance.

The analysis begins by placing $\widetilde{V_{o}}$ in terms of $\mathrm{L}$,

$$
\widetilde{V}_{o}=\frac{V_{D C} R_{L}}{Z_{o}} \sqrt{\frac{1-e^{-\alpha T_{d}}}{2 \alpha T_{d}}}=\frac{V_{D C} R_{L}}{\sqrt{\frac{L}{C}}} \sqrt{\frac{1-e^{-\alpha T_{d}}}{2 \alpha T_{d}}}=V_{D C} R_{L} \sqrt{\left(\frac{C}{L}\right)\left(\frac{1-e^{-\alpha T_{d}}}{2 \alpha T_{d}}\right)}
$$

Simplifying $\alpha T_{d}$ by substituting equations (3-4) and (3-12),

$$
\begin{aligned}
\alpha T_{d}=\alpha\left(\frac{2 \pi}{\omega_{d}}\right) & =\left(\frac{R_{\text {total }}}{2 L}\right)\left(\frac{2 \pi}{\sqrt{\frac{1}{L C}-\left(\frac{R_{\text {total }}}{2 L}\right)^{2}}}\right)=\frac{2 \pi}{\frac{2 L}{R_{\text {total }}} \sqrt{\frac{1}{L C}-\left(\frac{\left.R_{\text {total }}\right)^{2}}{2 L}\right.}}=\frac{2 \pi}{\left.\sqrt{\left(\frac{2 L}{R_{\text {total }}}\right)^{2}\left(\frac{1}{L C}-\left(\frac{R_{\text {total }}}{2 L}\right)^{2}\right)}-1\right)} \\
& =\frac{\left.\sqrt{\left(R_{\text {total }}^{2} C\right.}-1\right)}{\sqrt{\left.\frac{4 L}{R_{\text {total }}{ }^{2} C}-1\right)^{-\frac{1}{2}}}}
\end{aligned}
$$

Thus,

$$
\alpha T_{d}=2 \pi\left(\frac{4 L}{R_{\text {total }}^{2} C}-1\right)^{-\frac{1}{2}}
$$

Further simplifying by substituting equation (3-18) yields: 


$$
\begin{aligned}
& \widetilde{V}_{O}=V_{D C} R_{L} \sqrt{\left(\frac{C}{L}\right)\left(\frac{\left.\left.1-e^{-\left(2 \pi \left(\frac{4 L}{R_{\text {total }}{ }^{2} C}-1\right.\right.}\right)^{-\frac{1}{2}}\right)}{2\left(2 \pi\left(\frac{4 L}{R_{\text {total }^{2} C}}-1\right)^{-\frac{1}{2}}\right)}\right)} \\
& =\frac{V_{D C} R_{L}}{\sqrt{4 \pi}} \sqrt{\frac{C}{L} \sqrt{\frac{4 L}{R_{\text {total }^{2} C}}-1}\left(1-e^{\left.-2 \pi\left(\frac{4 L}{R_{\text {total }}^{2} C}-1\right)^{-\frac{1}{2}}\right)}\right)} \\
& =\frac{V_{D C} R_{L}}{\sqrt{4 \pi}} \sqrt{\sqrt{\frac{4 C}{L_{\text {total }^{2}}}-\frac{C^{2}}{L^{2}}}\left(1-e^{\left.-2 \pi\left(\frac{4 L}{R_{\text {total }}{ }^{2} C}-1\right)^{-\frac{1}{2}}\right)}\right.} \\
& =\frac{V_{D C} R_{L}}{\sqrt{4 \pi}}\left(\frac{4 C}{L R_{\text {total }}{ }^{2}}-\frac{C^{2}}{L^{2}}\right)^{\frac{1}{4}}\left(1-e^{-2 \pi\left(\frac{4 L}{R_{\text {total }}{ }^{2} C}-1\right)^{-\frac{1}{2}}}\right)^{\frac{1}{2}}
\end{aligned}
$$

Finding maximum output RMS voltage by taking derivative with respect to inductance,

$$
\frac{d \widetilde{V}_{o}}{d L}=\frac{d}{d L}\left\{\frac{V_{D C} R_{L}}{\sqrt{4 \pi}}\left(\frac{4 C}{L R_{\text {total }}^{2}}-\frac{C^{2}}{L^{2}}\right)^{\frac{1}{4}}\left(1-e^{-2 \pi\left(\frac{4 L}{R_{\text {total }}{ }^{2} C}-1\right)^{-\frac{1}{2}}}\right)^{\frac{1}{2}}\right\}
$$

Using U-V substitution to solve where,

$$
u=\frac{V_{D C} R_{L}}{\sqrt{4 \pi}}\left(\frac{4 C}{L R_{\text {total }}^{2}}-\frac{C^{2}}{L^{2}}\right)^{\frac{1}{4}}
$$

and, 


$$
\begin{aligned}
u^{\prime}=\frac{d u}{d L}=\frac{d}{d L}\{ & \left.\frac{V_{D C} R_{L}}{\sqrt{4 \pi}}\left(\frac{4 C}{L R_{\text {total }}{ }^{2}}-\frac{C^{2}}{L^{2}}\right)^{\frac{1}{4}}\right\} \\
= & \frac{V_{D C} R_{L}}{\sqrt{4 \pi}}\left(\frac{1}{4}\right)\left(\frac{2 C^{2}}{L^{3}}-\frac{4 C}{L^{2} R_{\text {total }}^{2}}\right)\left(\frac{4 C}{L R_{\text {total }}^{2}}-\frac{C^{2}}{L^{2}}\right)^{-\frac{3}{4}} \\
& =\frac{V_{D C} R_{L}}{\sqrt{4 \pi}}\left(\frac{C^{2}}{2 L^{3}}-\frac{C}{L^{2} R_{\text {total }}^{2}}\right)\left(\frac{4 C}{L R_{\text {total }}^{2}}-\frac{C^{2}}{L^{2}}\right)^{-\frac{3}{4}}
\end{aligned}
$$

Furthermore,

$$
v=\left(1-e^{-2 \pi\left(\frac{4 L}{R_{\text {total }}{ }^{2} C}-1\right)^{-\frac{1}{2}}}\right)^{\frac{1}{2}}
$$

and,

$$
v^{\prime}=\frac{d v}{d L}=\frac{d}{d L}\left\{\left(1-e^{-2 \pi\left(\frac{4 L}{R_{\text {total }}{ }^{2} C}-1\right)^{-\frac{1}{2}}}\right)^{\frac{1}{2}}\right\}
$$

Using $\mathrm{x}$-substitution to solve for $v^{\prime}$, where

$$
v=\left(1-e^{-x}\right)^{\frac{1}{2}}
$$

and,

$$
x=2 \pi\left(\frac{4 L}{R_{\text {total }}^{2} C}-1\right)^{-\frac{1}{2}}
$$

Finding the derivative of $\mathrm{x}$ with respect to $\mathrm{L}$ results in,

$$
\frac{d x}{d L}=2 \pi\left(-\frac{1}{2}\right)\left(\frac{4}{R_{\text {total }}^{2} C}\right)\left(\frac{4 L}{R_{\text {total }}^{2} C}-1\right)^{-\frac{3}{2}}=\left(\frac{-4 \pi}{R_{\text {total }}^{2} C}\right)\left(\frac{4 L}{R_{\text {total }}^{2} C}-1\right)^{-\frac{3}{2}}
$$

Furthermore,

$$
\frac{d v}{d x}=\frac{d}{d x}\left\{\left(1-e^{-x}\right)^{\frac{1}{2}}\right\}=\left(\frac{1}{2}\right)\left(e^{-x}\right)\left(1-e^{-x}\right)^{-\frac{1}{2}}=\left(\frac{e^{-x}}{2}\right)\left(1-e^{-x}\right)^{-\frac{1}{2}}
$$


Finally, $v^{\prime}$ is solved using the chain rule

$$
\begin{aligned}
v^{\prime}=\frac{d v}{d L}=\frac{d v}{d x} \frac{d x}{d L} & =\left[\left(\frac{e^{-x}}{2}\right)\left(1-e^{-x}\right)^{-\frac{1}{2}}\right]\left[\left(\frac{-4 \pi}{R_{\text {total }}^{2} C}\right)\left(\frac{4 L}{R_{\text {total }}^{2} C}-1\right)^{-\frac{3}{2}}\right] \\
= & \frac{\left(\frac{-4 \pi}{R_{\text {total }}^{2} C}\right)\left(\frac{e^{-x}}{2}\right)}{\left(1-e^{-x}\right)^{\frac{1}{2}}\left(\frac{4 L}{R_{\text {total }}^{2} C}-1\right)^{\frac{3}{2}}}
\end{aligned}
$$

Using product rule in combination with u-v substitution to determine,

$$
\begin{aligned}
\frac{d \widetilde{V}_{o}}{d L}=u v^{\prime}+v u^{\prime} & = \\
& =\left\{\frac{V_{D C} R_{L}}{\sqrt{4 \pi}}\left(\frac{4 C}{L R_{\text {total }}^{2}}-\frac{C^{2}}{L^{2}}\right)^{\frac{1}{4}}\right\}\left\{\frac{\left(\frac{-4 \pi}{R_{\text {total }}^{2} C}\right)\left(\frac{e^{-x}}{2}\right)}{\left(1-e^{-x}\right)^{\frac{1}{2}}\left(\frac{4 L}{R_{\text {total }}^{2} C}-1\right)^{\frac{3}{2}}}\right\} \\
& +\left\{\left(1-e^{-x}\right)^{\frac{1}{2}}\right\}\left\{\frac{V_{D C} R_{L}}{\sqrt{4 \pi}}\left(\frac{C^{2}}{2 L^{3}}-\frac{C}{L^{2} R_{\text {total }}^{2}}\right)\left(\frac{4 C}{L R_{\text {total }}^{2}}-\frac{C^{2}}{L^{2}}\right)^{-\frac{3}{4}}\right\}
\end{aligned}
$$

Setting expression equal to zero and cancelling like terms,

$$
\begin{gathered}
\frac{d \widetilde{V}_{o}}{d L}=0=\left\{\frac{4 C}{L R_{\text {total }}^{2}}-\frac{C^{2}}{L^{2}}\right\}\left\{\frac{\left(\frac{-4 \pi}{R_{\text {total }}{ }^{2} C}\right)\left(\frac{e^{-x}}{2}\right)}{\left(\frac{4 L}{R_{\text {total }}{ }^{2} C}-1\right)^{\frac{3}{2}}}\right\}+\left\{1-e^{-x}\right\}\left\{\frac{C^{2}}{2 L^{3}}-\frac{C}{L^{2} R_{\text {total }}^{2}}\right\} \\
{\left[\left(1-e^{-x}\right)\left(\frac{C^{2}}{2 L^{3}}-\frac{C}{L^{2} R_{\text {total }}^{2}}\right)=\frac{\left(\frac{4 C}{L R_{\text {total }}^{2}}-\frac{C^{2}}{L^{2}}\right)\left(\frac{2 \pi}{R_{\text {total }}{ }^{2} C}\right)\left(e^{-x}\right)}{\left(\frac{4 L}{R_{\text {total }}{ }^{2} C}-1\right)^{\frac{3}{2}}}\right] \frac{\left(e^{x}\right)}{\left(\frac{C^{2}}{2 L^{3}}-\frac{C}{L^{2} R_{\text {total }}{ }^{2}}\right)}}
\end{gathered}
$$




$$
\begin{aligned}
& e^{x}-1=\frac{\left(\frac{4 C}{L R_{\text {total }}^{2}}-\frac{C^{2}}{L^{2}}\right)\left(\frac{2 \pi}{R_{\text {total }}^{2} C}\right)}{\left(\frac{C^{2}}{2 L^{3}}-\frac{C}{L^{2} R_{\text {total }}{ }^{2}}\right)\left(\frac{4 L}{R_{\text {total }}^{2} C}-1\right)^{\frac{3}{2}}} \\
& e^{x}-1=\frac{\frac{L^{2}}{C^{2}}\left(\frac{4 C}{L R_{\text {total }}^{2}}-\frac{C^{2}}{L^{2}}\right)\left(\frac{2 \pi}{R_{\text {total }}^{2} C}\right)}{\frac{L^{2}}{C^{2}}\left(\frac{C^{2}}{2 L^{3}}-\frac{C}{L^{2} R_{\text {total }}{ }^{2}}\right)\left(\frac{4 L}{R_{\text {total }}{ }^{2} C}-1\right)^{\frac{3}{2}}} \\
& e^{x}-1=\frac{\left(\frac{4 L}{R_{\text {total }}^{2} C}-1\right)\left(\frac{2 \pi}{R_{\text {total }}{ }^{2} C}\right)}{\left(\frac{1}{2 L}-\frac{1}{R_{\text {total }}^{2} C}\right)\left(\frac{4 L}{R_{\text {total }}{ }^{2} C}-1\right)^{\frac{3}{2}}} \\
& e^{x}-1=\frac{\frac{R_{\text {total }}}{2}\left(\frac{2 \pi}{R_{\text {total }}{ }^{2} C}\right)}{\frac{R_{\text {total }}}{2}\left(\frac{1}{2 L}-\frac{1}{R_{\text {total }}{ }^{2} C}\right)\left(\frac{4 L}{R_{\text {total }}{ }^{2} C}-1\right)^{\frac{1}{2}}} \\
& e^{x}-1=\frac{R_{\text {total }} C}{R_{\text {total }} C} \frac{\left(\frac{\pi}{R_{\text {total }} C}\right)}{\left(\frac{1}{2 L}-\frac{1}{R_{\text {total }}{ }^{2} C}\right)\left(\frac{L}{C}-\left(\frac{R_{\text {total }}}{2}\right)^{2}\right)^{\frac{1}{2}}}
\end{aligned}
$$

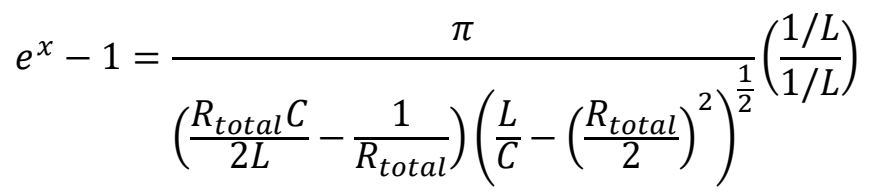

$$
\begin{aligned}
& e^{x}-1=\frac{\pi / L}{\left(\frac{R_{\text {total }} C}{2 L}-\frac{1}{R_{\text {total }}}\right)\left(\frac{1}{L C}-\left(\frac{R_{\text {total }}}{2 L}\right)^{2}\right)^{\frac{1}{2}}}
\end{aligned}
$$

Substituting in equation (3-6), thus simplifying the expression using the damped frequency, 


$$
e^{x}-1=\frac{\pi / L}{\left(\frac{R_{\text {total }} C}{2 L}-\frac{1}{R_{\text {total }}}\right) \omega_{d}}
$$

Substituting for $\mathrm{x}$ where,

$$
x=2 \pi\left(\frac{4 L}{R_{\text {total }}^{2} C}-1\right)^{-\frac{1}{2}}=\frac{\pi R_{\text {total }}}{L \omega_{d}}
$$

results in

$$
e^{\frac{\pi R_{\text {total }}}{L \omega_{d}}}-1=\frac{\pi / L}{\left(\frac{R_{\text {total }} C}{2 L}-\frac{1}{R_{\text {total }}}\right) \omega_{d}}
$$

Capacitance and inductance are related via the damped frequency; thus, the above equation is simplified by solving for the capacitance using equation (3-6),

$$
\begin{gathered}
\omega_{d}=\sqrt{\frac{1}{L C}-\left(\frac{R_{\text {total }}}{2 L}\right)^{2}} \\
\omega_{d}^{2}=\frac{1}{L C}-\left(\frac{R_{\text {total }}}{2 L}\right)^{2} \\
\omega_{d}^{2}+\left(\frac{R_{\text {total }}}{2 L}\right)^{2}=\frac{1}{L C} \\
\frac{\omega_{d}^{2} L+\frac{R_{\text {total }}{ }^{2}}{4 L}=\frac{1}{C}}{1} \\
\frac{\omega_{d}^{2} L+\frac{R_{\text {total }}^{2}}{4 L}}{\left(2 \omega_{d} L\right)^{2}+R_{\text {total }}^{2}}
\end{gathered}
$$

Substituting equation (3-8) into the derived expression and simplifying, 


$$
\begin{gathered}
e^{\frac{\pi R_{\text {total }}}{L \omega_{d}}}-1=\frac{\pi / L}{\left(\frac{R_{\text {total }}}{2 L}\left(\frac{4 L}{\left(2 \omega_{d} L\right)^{2}+R_{\text {total }}{ }^{2}}\right)-\frac{1}{R_{\text {total }}}\right) \omega_{d}} \\
e^{\frac{\pi R_{\text {total }}}{L \omega_{d}}}-1=\frac{R_{\text {total }}}{R_{\text {total }}\left(\frac{2 R_{\text {total }}}{\left(2 \omega_{d} L\right)^{2}+R_{\text {total }}{ }^{2}}-\frac{1}{R_{\text {total }}}\right) \omega_{d}} \\
e^{\frac{\pi R_{\text {total }}}{L \omega_{d}}-1}=\frac{\pi R_{\text {total }}}{L \omega_{d}\left(\frac{2 R_{\text {total }}{ }^{2}}{\left(2 \omega_{d} L\right)^{2}+R_{\text {total }}{ }^{2}}-1\right)} \\
K_{2}=\frac{\pi R_{\text {total }}}{L \omega_{d}-1} \\
L \omega_{d}\left(\frac{\pi R_{\text {total }}}{\left(2 \omega_{d} L\right)^{2}+R_{\text {total }}^{2}}-1\right) \\
K_{1}^{2}
\end{gathered}
$$

The derived expression does not have an elementary algebraic solution. Instead, a solution is determined by finding the intersection between equations (3-20) and (3-21). Additionally, for a real solution to exist the damped frequency, $\omega_{d}$, must be greater than the attenuation factor, $\alpha$. This is evident by equation (3-21) where a solution must exist below the asymptote (i.e. $L=\frac{R_{t o t a l}}{2 \omega_{d}}$ ), since all values must be real and positive. Thus,

$$
L<\frac{R_{\text {total }}}{2 \omega_{d}}
$$

\subsection{Peak Output Current/Voltage}

Peak current is a system parameter concerning component ratings. Peak current occurs every half-cycle, where a positive peak current occurs for the first half-cycle and a negative peak current occurs for the second half-cycle. The magnitude of each peak is equivalent by symmetry; 
thus, only the peak current of the positive cycle is derived. A sinusoid exhibits peaking at a quarter wave; however, this is not true for the damped frequency sine wave of the SLR inverter. Consequently, it becomes necessary to determine the time in which the peak occurs. Peak time is derived by identifying the time at which the output current is at a maximum. This derivation is performed below:

$$
\frac{d i_{o}(t)}{d t}=\frac{d}{d t}\left[\left(\frac{V_{D C}}{Z_{e f f}}\right) *\left(e^{-\alpha t}\right) *\left(\sin \omega_{d} t\right)\right]=\left(\frac{V_{D C}}{Z_{e f f}}\right)\left(\frac{d}{d t}\left[\left(e^{-\alpha t}\right) *\left(\sin \omega_{d} t\right)\right]\right)
$$

By the product rule,

$$
\frac{d i_{o}(t)}{d t}=\left(\frac{V_{D C}}{Z_{e f f}}\right)\left(\omega_{d} e^{-\alpha t} \cos \left(\omega_{d} t\right)-\alpha e^{-\alpha t} \sin \left(\omega_{d} t\right)\right)
$$

Finding $t_{\max }$ by setting $\frac{d I_{o}(t)}{d t}=0$,

$$
\begin{gathered}
0=\left(\frac{V_{D C}}{Z_{e f f}}\right)\left(\omega_{d} e^{-\alpha t_{\max }} \cos \left(\omega_{d} t_{\max }\right)-\alpha e^{-\alpha t_{\max }} \sin \left(\omega_{d} t_{\max }\right)\right) \\
0=\omega_{d} e^{-\alpha t_{\max }} \cos \left(\omega_{d} t_{\max }\right)-\alpha e^{-\alpha t_{\max } \sin \left(\omega_{d} t_{\max }\right)} \\
\omega_{d} \cos \left(\omega_{d} t_{\max }\right)=\alpha \sin \left(\omega_{d} t_{\max }\right) \\
\frac{\sin \left(\omega_{d} t_{\max }\right)}{\cos \left(\omega_{d} t_{\max }\right)}=\frac{\omega_{d}}{\alpha}
\end{gathered}
$$

By tangent quotient identity,

$$
\begin{gathered}
\tan \left(\omega_{d} t_{\text {max }}\right)=\frac{\omega_{d}}{\alpha} \\
\omega_{d} t_{\text {max }}=\tan ^{-1}\left(\frac{\omega_{d}}{\alpha}\right)
\end{gathered}
$$

Thus, 


$$
t_{\text {max }}=\frac{\tan ^{-1}\left(\frac{\omega_{d}}{\alpha}\right)}{\omega_{d}}
$$

Combining equations (3-11) and (3-23),

$$
\begin{aligned}
i_{\text {peak }}=i_{o}\left(t_{\text {max }}\right) & =\left[\frac{V_{D C}}{Z_{\text {eff }}}\right] *\left[e^{-\frac{\alpha}{\omega_{d}} \tan ^{-1}\left(\frac{\omega_{d}}{\alpha}\right)}\right]\left[\sin \left(\omega_{d} \frac{\tan ^{-1}\left(\frac{\omega_{d}}{\alpha}\right)}{\omega_{d}}\right)\right] \\
& =\left[\frac{V_{D C}}{Z_{\text {eff }}}\right] *\left[e^{-\frac{\alpha}{\omega_{d}} \tan ^{-1}\left(\frac{\omega_{d}}{\alpha}\right)}\right]\left[\sin \left(\tan ^{-1}\left(\frac{\omega_{d}}{\alpha}\right)\right)\right]
\end{aligned}
$$

Using forward inverse identity to simplify,

$$
\begin{aligned}
i_{\text {peak }}=\left[\frac{V_{D C}}{Z_{\text {eff }}}\right] *\left[e^{-\frac{\alpha}{\omega_{d}} \tan ^{-1}\left(\frac{\omega_{d}}{\alpha}\right)}\right]\left[\frac{\frac{\omega_{d}}{\alpha}}{\sqrt{1+\left(\frac{\omega_{d}}{\alpha}\right)^{2}}}\right]=\left[\frac{V_{D C} \omega_{d}}{\left.\alpha Z_{\text {eff }} \sqrt{1+\left(\frac{\omega_{d}}{\alpha}\right)^{2}}\right] *\left[e^{-\frac{\alpha}{\omega_{d}} \tan ^{-1}\left(\frac{\omega_{d}}{\alpha}\right)}\right]}\right. \\
=\left[\frac{V_{D C} \omega_{d}}{Z_{e f f} \sqrt{\alpha^{2}+\omega_{d}^{2}}}\right] *\left[e^{-\frac{\alpha}{\omega_{d}} \tan ^{-1}\left(\frac{\omega_{d}}{\alpha}\right)}\right]=\left[\frac{V_{D C} \omega_{d}}{Z_{e f f} \omega_{o}}\right] *\left[e^{-\frac{\alpha}{\omega_{d}} \tan ^{-1}\left(\frac{\omega_{d}}{\alpha}\right)}\right]
\end{aligned}
$$

Further simplifying by substituting equations (3-5), (3-6), and (3-8)

$$
\begin{gathered}
i_{\text {peak }}=\left[\frac{V_{D C} \sqrt{\frac{1}{L C}-\left(\frac{R_{\text {total }}}{2 L}\right)^{2}}}{\left.\sqrt{\frac{L}{C}-\left(\frac{\left.R_{\text {total }}\right)^{2}}{2} \sqrt{\frac{1}{L C}}\right.}\right]\left(\frac{L}{L}\right) *\left[e^{-\frac{\alpha}{\omega_{d}} \tan ^{-1}\left(\frac{\omega_{d}}{\alpha}\right)}\right]}\right. \\
=\left[\frac{V_{D C} \sqrt{\frac{L}{C}-\left(\frac{R_{\text {total }}}{2}\right)^{2}}}{\sqrt{\frac{L}{C}-\left(\frac{R_{\text {total }}}{2}\right)^{2}} \sqrt{\frac{L}{C}}}\right] *\left[e^{-\frac{\alpha}{\omega_{d}} \tan ^{-1}\left(\frac{\omega_{d}}{\alpha}\right)}\right]=\left[\frac{V_{D C}}{Z_{o}}\right] *\left[e^{\left.-\frac{\alpha}{\omega_{d}} \tan ^{-1}\left(\frac{\omega_{d}}{\alpha}\right)\right]}\right. \\
i_{\text {peak }}=\left[\frac{V_{D C}}{Z_{o}}\right] *\left[e^{-\frac{\alpha}{\omega_{d}} \tan ^{-1}\left(\frac{\omega_{d}}{\alpha}\right)}\right]
\end{gathered}
$$

Ohms law then determines the peak load voltage as, 


$$
v_{\text {peak }}=i_{\text {peak }} R_{L}=\left[\frac{V_{D C} R_{L}}{Z_{o}}\right] *\left[e^{-\frac{\alpha}{\omega_{d}} \tan ^{-1}\left(\frac{\omega_{d}}{\alpha}\right)}\right]
$$

\subsection{Efficiency}

The SLR inverter has the potential of high efficiency. The theoretical efficiency of the SLR inverter is determined based on zero current switching (ZCS) for both turn-off and turn-on; thus, switching losses are neglected in the derivation of efficiency. Conduction losses include load resistance, capacitor series resistance, inductor winding resistance, and switch on resistance. Although there are two switches, the combined losses between the two switches is equivalent to a series switch that is constantly conducting the output current. This assumes both switch on resistances are equivalent and the duty cycle of each switch is $50 \%$. Theoretical calculations for efficiency are determined below.

$$
\begin{aligned}
\eta=\frac{P_{\text {out }}}{P_{\text {in }}} * 100 \% & =\frac{P_{\text {out }}}{P_{\text {out }}+P_{L}+P_{C}+P_{D S(\text { on })}} 100 \% \\
& =\frac{{\widetilde{I_{o}}}^{2} R_{L}}{\widetilde{I}_{o}^{2} R_{L}+{\widetilde{I_{o}}}^{2} R_{S}+{\widetilde{I_{o}}}^{2} R_{\text {esr }}+{\widetilde{I_{o}}}^{2} R_{D S(\text { on })}} 100 \% \\
& =\frac{R_{L}}{R_{L}+R_{S}+R_{\text {esr }}+R_{D S(\text { on })}} 100 \%
\end{aligned}
$$

Substituting equation (3-2),

$$
\eta=\frac{R_{L}}{R_{\text {total }}} 100 \%
$$

Equation (3-26) demonstrates that the ratio between the load resistance and total series resistance determines the efficiency. High efficiencies may be attained by selecting a large ratio between load resistance and inherent parasitic resistances. This topic is discussed and analyzed in the following chapter. 


\subsection{Component Sizing}

In order to design a practical SLR inverter, consideration of component ratings is critical. This section describes the necessary component ratings for the inductor, capacitor, and switch. The component rating calculations are not specific to the optimum inductor selection design method. Once component values are determined (i.e. capacitance, inductance) these equations are used to realize component ratings.

\subsubsection{Inductor}

Inductor component ratings rely on rated current and saturation current. Rated current describes the allowable RMS current that ensures the inductor will not overheat. Saturation current describes the maximum allowable current through the inductor in order to maintain linear operation. Equations (3-16) and (3-24) define the RMS and peak inductor current respectively. These values must always be less than the inductor current ratings, as shown below.

$$
I_{L r}>\widetilde{I_{o}}
$$

Substituting equation (3-16),

$$
I_{L r}>\frac{V_{D C}}{Z_{o}} \sqrt{\frac{1-e^{-\alpha T_{d}}}{2 \alpha T_{d}}}
$$

Also, the saturation current must be greater than the peak current, as shown below.

$$
I_{\text {sat }}>i_{\text {peak }}
$$

Substituting equation (3-24),

$$
I_{s a t}>\left[\frac{V_{D C}}{Z_{o}}\right] *\left[e^{-\frac{\alpha}{\omega_{d}} \tan ^{-1}\left(\frac{\omega_{d}}{\alpha}\right)}\right]
$$




\subsubsection{Capacitor}

Capacitor component ratings rely on the peak voltage imposed on the capacitor. This is known as the capacitor $\mathrm{DC}$ voltage rating, $\mathrm{V}_{\mathrm{DCr}}$. The $\mathrm{DC}$ voltage rating of the capacitor must always be greater than the peak voltage across the capacitor; thus, it becomes necessary to calculate capacitor peak voltage. Capacitor peak voltage occurs at half a period, since a positive step input charges the capacitor to a maximum value. Using this knowledge in conjunction with the capacitor charging equation, capacitor peak voltage across the capacitor is derived. In addition, the current through the capacitor is equivalent to the current through the load; thus, equation (3-16) is used in the derivation, as shown below.

$$
\begin{gathered}
V_{D C r}>\frac{1}{C} \int_{0}^{T_{d} / 2} i_{o}(t) d t \\
V_{D C r}>\frac{1}{C} \int_{0}^{T_{d} / 2}\left[\frac{V_{D C}}{Z_{e f f}}\right] *\left[e^{-\alpha t}\right] *\left[\sin \omega_{d} t\right] d t
\end{gathered}
$$

Using a table of integrals, [28], to solve results in,

$$
\begin{gathered}
V_{D C r}>\frac{1}{C}\left[\frac{V_{D C}}{Z_{e f f}}\right]\left[\left.\frac{e^{-a t}}{\omega_{d}^{2}+\alpha^{2}}\left(-\alpha \sin \omega_{d} t-\omega_{d} \cos \omega_{d} t\right)\right|_{0} ^{T_{d} / 2}\right] \\
V_{D C r}>\frac{1}{C}\left[\frac{V_{D C}}{Z_{e f f}\left(\omega_{d}^{2}+\alpha^{2}\right)}\right]\left[e^{-\frac{a T_{d}}{2}}\left(-\alpha \sin \omega_{d} \frac{T_{d}}{2}+\omega_{d} \cos \omega_{d} \frac{T_{d}}{2}\right)\right. \\
\left.-e^{0}\left(-\alpha \sin 0-\omega_{d} \cos 0\right)\right] \\
V_{D C r}>\frac{1}{C}\left[\frac{V_{D C}}{Z_{e f f}\left(\omega_{d}^{2}+\alpha^{2}\right)}\right]\left[e^{-\frac{a T_{d}}{2}}\left(\omega_{d}\right)+\omega_{d}\right]
\end{gathered}
$$

where $\omega_{o}{ }^{2}=\omega_{d}{ }^{2}+\alpha^{2}$, 


$$
V_{D C r}>\frac{1}{C}\left[\frac{V_{D C} \omega_{d}}{Z_{e f f} \omega_{o}^{2}}\right]\left[e^{-\frac{a T_{d}}{2}}+1\right]
$$

Substituting equations (3-5), (3-6), and (3-8),

$$
\begin{gathered}
V_{D C r}>\frac{V_{D C}}{C}\left[\frac{\sqrt{\frac{1}{L C}-\left(\frac{R_{\text {total }}}{2 L}\right)^{2}}}{\sqrt{\frac{L}{C}-\left(\frac{\left.R_{\text {total }}\right)^{2}}{2}\left(\frac{1}{L C}\right)\right.}}\right]\left(e^{\left.-\frac{a T_{d}}{2}+1\right)}\right. \\
V_{D C r}>\frac{V_{D C}}{C}\left[\frac{\sqrt{\frac{1}{L C}-\left(\frac{R_{\text {total }}}{2 L}\right)^{2}}}{\sqrt{\frac{1}{L C}-\left(\frac{R_{\text {total }}}{2 L}\right)^{2}}\left(\frac{1}{C}\right)}\right]\left(e^{\left.-\frac{a T_{d}}{2}+1\right)}\right. \\
V_{D C r}>V_{D C}\left(e^{-\frac{a T_{d}}{2}}+1\right)
\end{gathered}
$$

\subsubsection{Switch}

Switch component ratings are determined by the maximum voltage and RMS current. The maximum voltage across each switch is the same as the DC input voltage. The RMS current rating of the switch must be greater than the RMS current through the switch. Since the switch conducts only half a cycle, the RMS current is proportional to the output RMS current given by equation (3-16). The derivations for these ratings are shown below.

$$
V_{S w(\max )}>V_{D C}
$$

The RMS current through the switch is proportional to the square root of the duty cycle. The duty cycle is assumed to be $50 \%$ since the switch is only conducting for half of the period.

$$
\tilde{I}_{s w r}>\widetilde{I}_{o} \sqrt{D}
$$




$$
\begin{gathered}
\tilde{I}_{s w r}>\tilde{I}_{o} \sqrt{1 / 2} \\
\tilde{I}_{s w r}>\frac{\tilde{I}_{o}}{\sqrt{2}}
\end{gathered}
$$

Substituting equation (3-16) results in,

$$
\tilde{I}_{s w r}>\frac{V_{D C}}{Z_{o}} \sqrt{\frac{1-e^{-\alpha T_{d}}}{4 \alpha T_{d}}}
$$




\section{Chapter 4: SLR Inverter Design}

\subsection{Design Goals}

An SLR inverter prototype is designed using the optimum inductance selection method. The prototype supplies a $10 \mathrm{~W}$ load. The resonant frequency is chosen to be $70 \mathrm{~Hz}$ to limit magnetic component sizing. The load resistance is set to $4.5 \Omega$, and $0.5 \Omega$ parasitic series resistance is assumed; thus, the total path resistance is $5 \Omega$. A solution to equations (3-20) and (321) result in an optimum inductance of $5.62 \mathrm{mH}$. Note that the solution meets the criteria set by equation (3-22). Furthermore, equation (3-19) results in an optimum capacitance of $454.7 \mu \mathrm{F}$. Equation (3-17) is used to solve for the required input voltage based on the parameters specified above. The results show $18.7 \mathrm{~V}_{\mathrm{DC}}$ is required for the input voltage to supply the $10 \mathrm{~W}$ load. The design parameters are summarized in Table 4-1.

Table 4-1: Design Parameters Summary

\begin{tabular}{|l|r|}
\hline Frequency $(\mathrm{Hz})$ & 70.00 \\
\hline Input Voltage $\left(\mathbf{V}_{\mathrm{DC}}\right)$ & 18.70 \\
\hline $\mathbf{L}_{\text {opt }}(\mathbf{m H})$ & 5.62 \\
\hline $\mathbf{C}_{\text {opt }}(\boldsymbol{\mu} \mathrm{F})$ & 454.70 \\
\hline $\mathbf{R}_{\text {load }}(\boldsymbol{\Omega})$ & 4.50 \\
\hline $\mathbf{R}_{\text {par }}(\boldsymbol{\Omega})$ & 0.50 \\
\hline $\mathbf{R}_{\text {total }}(\boldsymbol{\Omega})$ & 5.00 \\
\hline Output Power $(\mathbf{W})$ & 10.00 \\
\hline
\end{tabular}

\subsection{Controls}

The IRS2153 self-oscillating half-bridge driver IC is selected to provide gate drive for both MOSFETs [30]. Additionally, IRS2153 provides self-oscillating capabilities by selecting appropriate resistor and capacitor values. The frequency is expected to deviate with load, thus a variable resistor is used to tune the desired drive frequency. IRS2153 drives the two MOSFETs with a $50 \%$ duty cycle and a 1.1 us dead time. A bootstrap capacitor is also selected to provide hold-up time between switching states for driving the high side switch. The typical connection diagram is demonstrated in Figure 4-1, which is abstracted from the datasheet [30]. 


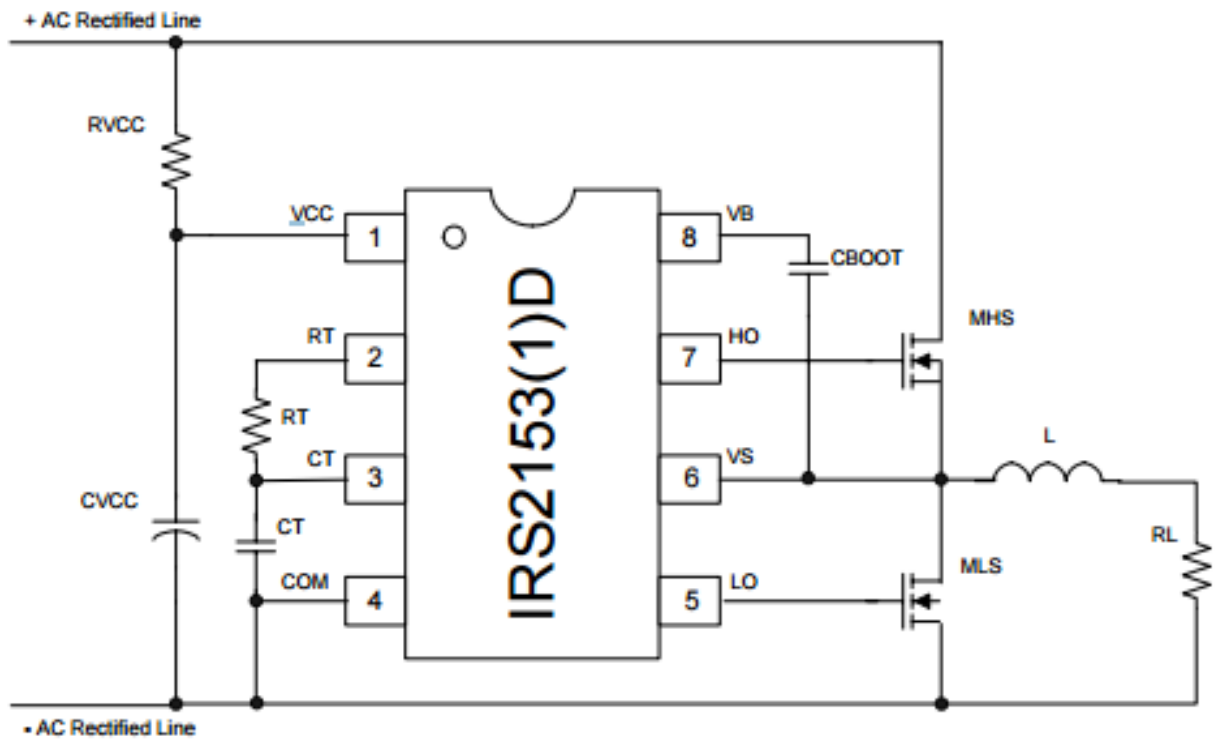

Figure 4-1: IRS2153 Typical connection diagram [30]

A frequency range between $50 \mathrm{~Hz}$ and $100 \mathrm{~Hz}$ is desired to provide frequency tuning range during testing. Equation (4-1) is stated in the IRS2153 datasheet to determine component values for the desired frequency range. Based on the desired frequency range, $\mathrm{CT}$ is set to $10 \mathrm{uF}$ and $\mathrm{RT}$ ranges from $500 \Omega$ to $1 \mathrm{k} \Omega$.

$$
f \sim \frac{1}{1.453 x R T x C T}
$$

The bootstrap capacitor is used to drive the high-side MOSFET. The bootstrap capacitor charges while the low side switch is on, and discharges on the following cycle to drive the high side MOSFET. An appropriate value capacitor must be chosen to ensure the energy in the capacitor is sufficient to drive the high side switch. Application note [31], describes the required capacitance based on switching frequency, supply voltage, capacitor leakage, and MOSFET characteristics. The information presented in [31] is shown in Figure 4-2. Bootstrap capacitance is selected for the worst case frequency of operation of $50 \mathrm{~Hz}$. A $12 \mathrm{~V}$ voltage supply is used to power the IRS2153. The IRS2153 contains an intrinsic diode and parameters are abstracted from 
the datasheet [30]. $\mathrm{V}_{\min }$ is assumed to be $0 \mathrm{~V}$, since the capacitor is expected to discharge completely. $\mathrm{V}_{\mathrm{LS}}$ is assumed to be $0 \mathrm{~V}$, since $\mathrm{ZCS}$ would result in a $0 \mathrm{~V}$ drop across the low side switch before switching to the next cycle. The remaining parameters are found in the data sheets [28] and [30], with the exception of the capacitor leakage current which is assumed to be $10 \mathrm{uA}$. The resulting capacitance calculation yields $0.382 \mathrm{uF}$; thus, a capacitance of $4.7 \mathrm{uF}$ is selected to ensure the energy in the capacitor is sufficient to drive the high side MOSFET. The design values of the controller are demonstrated in Table 4-2.

$$
C \geq \frac{2\left[2 Q_{g}+\frac{I_{q b s(\max )}}{f}+Q_{l s}+\frac{I_{C b s(l e a k)}}{f}\right]}{V_{c c}-V_{f}-V_{L S}-V_{M i n}}
$$

where:

$\mathrm{Qg}_{\mathrm{g}}=$ Gate charge of high-side FET

$f=$ frequency of operation

$\mathrm{I}_{\mathrm{Cbs}(\mathrm{leak})}=$ bootstrap capacitor leakage current

$\mathrm{I}_{\mathrm{qbs}(\max )}=$ Maximum $\mathrm{V}_{\mathrm{BS}}$ quiescent current

$V_{C C}=$ Logic section voltage source

$V_{f}=$ Forward voltage drop across the bootstrap diode

$V_{L S}=$ Voltage drop across the low-side FET or load

$\mathrm{V}_{\text {Min }}=$ Minimum voltage between $\mathrm{V}_{\mathrm{B}}$ and $\mathrm{V}_{\mathrm{S}}$.

$\mathrm{Q}_{\mathrm{Is}}=$ level shift charge required per cycle (typically $5 \mathrm{nC}$ for $500 \mathrm{~V} / 600 \mathrm{~V}$ MGDs and $20 \mathrm{nC}$ for 1200 V MGDs)

Figure 4-2: Required bootstrap capacitance [31]

Table 4-2: IRS2153 self-oscillating controller design values

\begin{tabular}{|l|r|}
\hline $\mathbf{V}_{\mathrm{CC}}\left(\mathbf{V}_{\mathrm{DC}}\right)$ & 12 \\
\hline $\mathbf{C}_{\text {boot }}(\mathbf{u F})$ & 110 \\
\hline RT $(\Omega)$ & $(0,1000)$ \\
\hline CT (uF) & 10 \\
\hline
\end{tabular}




\subsection{Switch}

The switch is designed based on the $10 \mathrm{~W}$ load and $18.7 \mathrm{~V}$ input voltage. Since the maximum voltage stress on the switch is equivalent to the input voltage, the maximum rated voltage is chosen to be $60 \mathrm{~V}$ by equation (3-30). Additionally, the maximum RMS current of each switch is determined to be $1.05 \mathrm{~A}$, given by equation (3-31); thus, a current rating of $110 \mathrm{~A}$ is chosen to avoid excessively heating the switches. Based on the switch maximum required component values the IRFB7540 MOSFET is selected. Summary parameters for the IRFB7540 are demonstrated in Table 4-3, which are abstracted from the datasheet [28].

\section{Table 4-3: IRFB7540 MOSFET Parameters}

\begin{tabular}{|l|r|}
\hline $\mathbf{V}_{\text {DSS }}\left(\mathbf{V}_{\mathrm{DC}}\right)$ & 60 \\
\hline $\mathbf{I}_{\mathrm{D}}\left(\mathbf{A}_{\text {rms }}\right)$ & 110 \\
\hline$R_{\mathrm{DS} \text { (on) }}(\mathbf{m} \Omega)$ & 5.1 \\
\hline
\end{tabular}

\subsection{Inductor}

The inductor is designed for a $5.62 \mathrm{mH}$ inductance capable of sustaining a $10 \mathrm{~W}$ output. Two inductors are wound to achieve the $5.62 \mathrm{mH}$ inductance and are placed in series. The allowable saturation current is determined to be greater than $2.42 \mathrm{~A}$, by equation (3-28). The inductance and series resistance of each inductor is measured using an Instek LCR-819 meter with a $1.25 \mathrm{~V}_{\mathrm{RMS}} \mathrm{AC}$ voltage and a $70 \mathrm{~Hz}$ frequency. Each inductor is designed to be $2.81 \mathrm{mH}$; however, winding inaccuracies result in a slight drift from the design value.

The saturation current of both inductors is measured using the half-bridge circuit consisting of the IRS2153 controller and two IRFB7540 MOSFETs. A square wave is applied to each inductor and current is measured via a $100 \mathrm{~m} \Omega$ resistor. A triangular current wave is observed through the inductor. The input voltage is increased until the triangular current becomes non-linear. Due to power supply limitations, the current through each inductor is limited to $3 \mathrm{~A}$. 
Each inductor demonstrates linearity up to $3 \mathrm{~A}$; thus, both inductors are rated to $3 \mathrm{~A}$ saturation current, which meets specifications. Summary of inductor parameters are shown below.

Table 4-4: Measured inductor values

\begin{tabular}{|l|r|}
\hline $\mathbf{L}_{1}(\mathbf{m H})$ & 2.84 \\
\hline$R_{\text {s1 }}(\mathbf{m} \Omega)$ & 95.74 \\
\hline $\mathbf{I}_{\text {sat1 }}(\mathbf{A})$ & 3.00 \\
\hline $\mathbf{L}_{\mathbf{2}}(\mathbf{m H})$ & 2.88 \\
\hline$R_{\mathrm{s} 2}(\mathbf{m} \Omega)$ & 100.28 \\
\hline $\mathbf{I}_{\text {sat2 }}(\mathbf{A})$ & 3.00 \\
\hline
\end{tabular}

\subsection{Capacitor}

The capacitor is designed for a $454.7 \mathrm{uF}$ capacitance; however, due to component tolerance a $458.38 \mathrm{uF}$ capacitance is measured. Additionally, the series resistance of the capacitor, $\mathrm{R}_{\mathrm{esr}}$, is measured to be $10.9 \mathrm{~m} \Omega$. Capacitor measurements are performed using the Instek LCR819 meter with a $1.25 \mathrm{~V}_{\mathrm{RMS}} \mathrm{AC}$ voltage at a $70 \mathrm{~Hz}$ frequency. Based on equation (3-29), the $\mathrm{DC}$ rating of the capacitor must be greater than $19.5 \mathrm{~V}$; thus, a $63 \mathrm{~V}$ rated capacitor is used to ensure the DC rating is not exceeded. Measured capacitor values are summarized in Table 4-5.

\section{Table 4-5: Capacitor rating and measured values}

\begin{tabular}{|l|r|}
\hline $\mathbf{C}(\mathbf{u F})$ & 454.7 \\
\hline$R_{\text {esr }}(\mathbf{m} \Omega)$ & $10.9 \mathrm{~m}$ \\
\hline $\mathbf{V}_{\mathrm{DCr}}(\mathbf{V})$ & 63 \\
\hline
\end{tabular}

\subsection{Design summary}

An SLR inverter prototype is constructed based on the design parameters developed in this chapter. Design is based on $70 \mathrm{~Hz}$ operation; however, component tolerance results in a deviation from the expected frequency. Characteristics of the designed inverter are demonstrated in the following chapter. The SLR inverter is assembled on proto-board and demonstrated in Figure 4-3. 


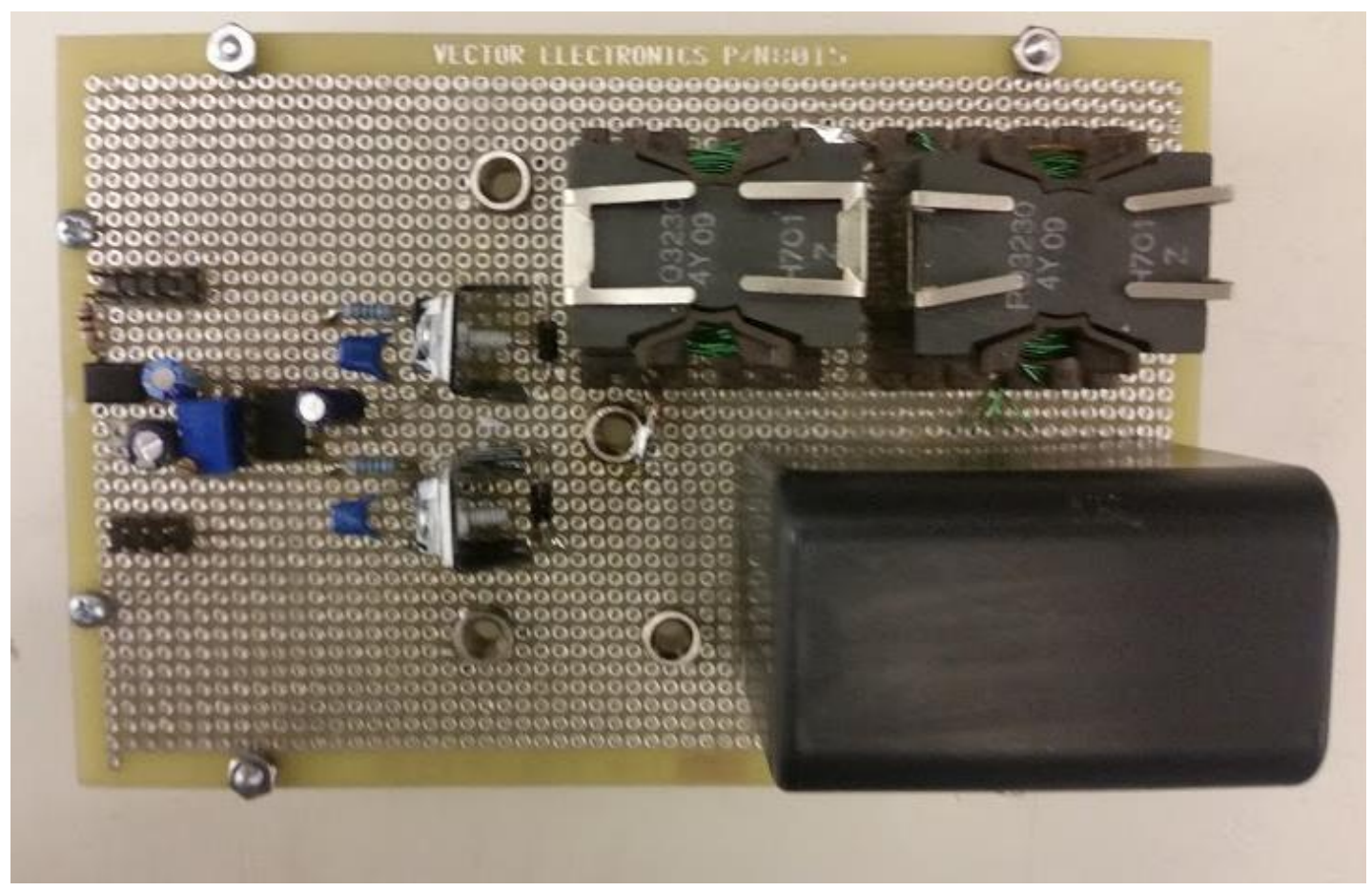

Figure 4-3: 70Hz SLR inverter prototype 


\section{Chapter 5: Hardware and Simulation Results}

\subsection{SLR Inverter Simulation Model}

Simulations are performed to verify the proposed design strategy, mathematical model, and prototype. The mathematical model disregards initial conditions in component values. These initial conditions are expected to alter performance of the SLR inverter, as predicted by the mathematical model. In practice, initial conditions are present due to the switching of the halfbridge. These effects are demonstrated using an LTSpice simulation model shown in Figure 5-1.

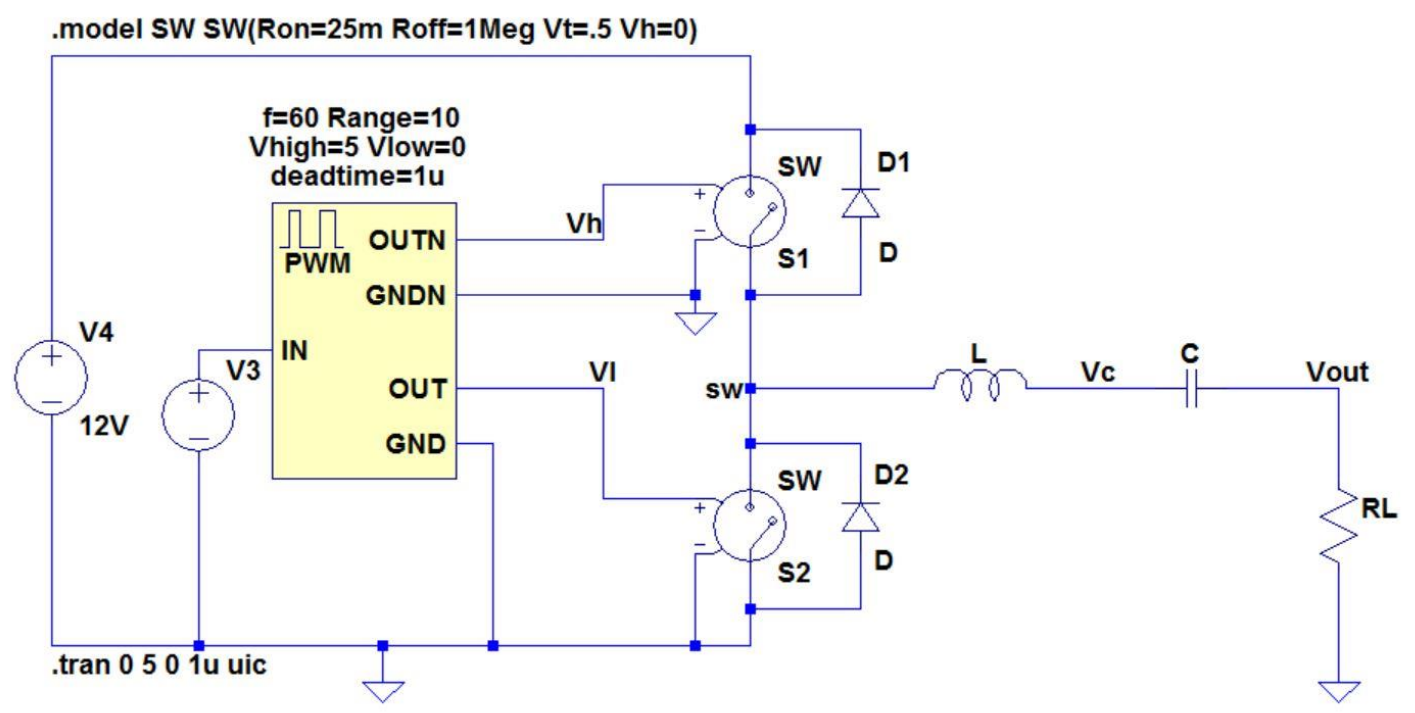

Figure 5-1: LTSpice simulation model of SLR inverter

The simulation model is composed of components previously defined by the SLR inverter topology, which include two switches, inductor, capacitor, and load resistor. The switch model is shown at the top of Figure 5-1 with the on resistance is set to a typical value of $25 \mathrm{~m} \Omega$. The series resistance of the capacitor and inductor are adjusted to achieve a desired total series path resistance. Anti-parallel diodes are placed across the switches to provide a freewheeling path for current during switching transitions. Even though ZCS is achieved with the SLR inverter, a finite amount of current is expected during transitions, due to the inability of switching accurately during zero current. Thus, the anti-parallel diodes clamp voltage spikes generated by the 
freewheeling current of the inductor. Figure 5-2 demonstrates ZCS characteristics of the SLR inverter.

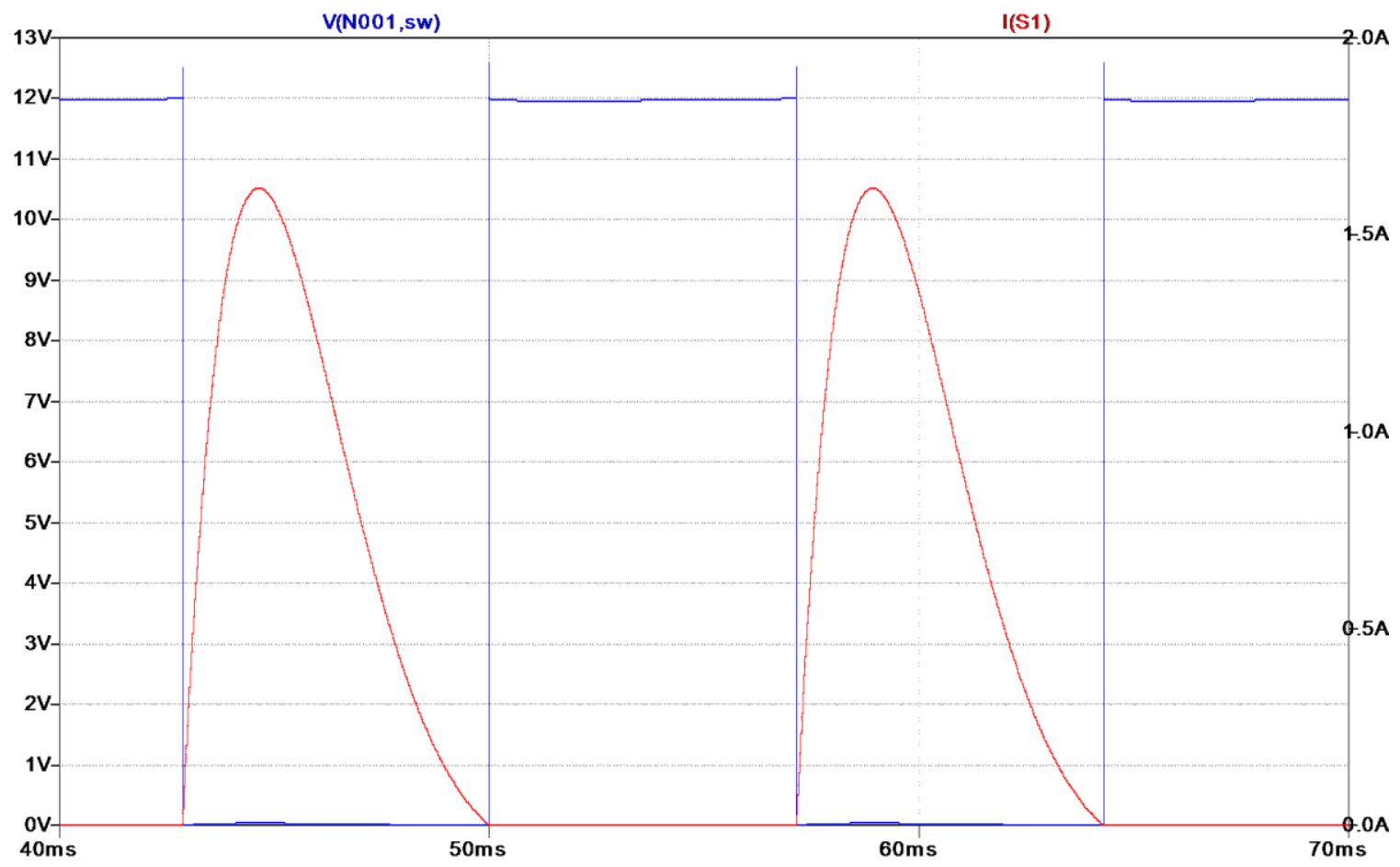

Figure 5-2: SLR inverter switch current and voltage demonstrating ZCS

In addition to the previously stated components, a control block regulates the control signals of the half-bridge switches. The control block's controllable parameters include: deadtime, high output voltage, low output voltage, frequency, and duty cycle. The control block provides programmable dead-time, thus ensuring that the two switches do not conduct at the same instant. Duty cycle is set by the ratio between the variable "range" and the voltage at the "IN" port. The duty cycle is set to $50 \%$ by specifying a 10 for the range value and applying $5 \mathrm{~V}$ at the "IN" port. The Spice model for the control block is shown in Appendix A.

Simulations are performed to obtain the RMS output current versus inductance. For each inductor value, a corresponding capacitance is selected so that the frequency of the system remains constant, based on equation (3-19). The RMS output current is measured in simulation for the first half-cycle and in steady state conditions. It is expected that the RMS of the first half- 
cycle will correspond to the mathematical model, since initial conditions are zero. The steady state value is evaluated to determine the accuracy of the mathematical model. Initial conditions for the load and inductor are zero on cycle to cycle basis; however, capacitor voltage is charged to a finite value before switching to the next cycle. This creates an initial voltage on the capacitor for the following cycle. The absence of initial conditions in the mathematical model fails to account for capacitor voltage at a switching transition. Figure 5-3 demonstrates the capacitor voltage, switch node, and the output voltage waveforms for the first 2 cycles. On the second cycle (approximately $28 \mathrm{~ms}$ ) switching occurs while the capacitor is charged, thus presenting an initial condition for the following cycle. This causes the output current to rise above the current predicted by the mathematical model. This effect is investigated by comparing the mathematical model with the simulated steady state behavior of the SLR inverter.

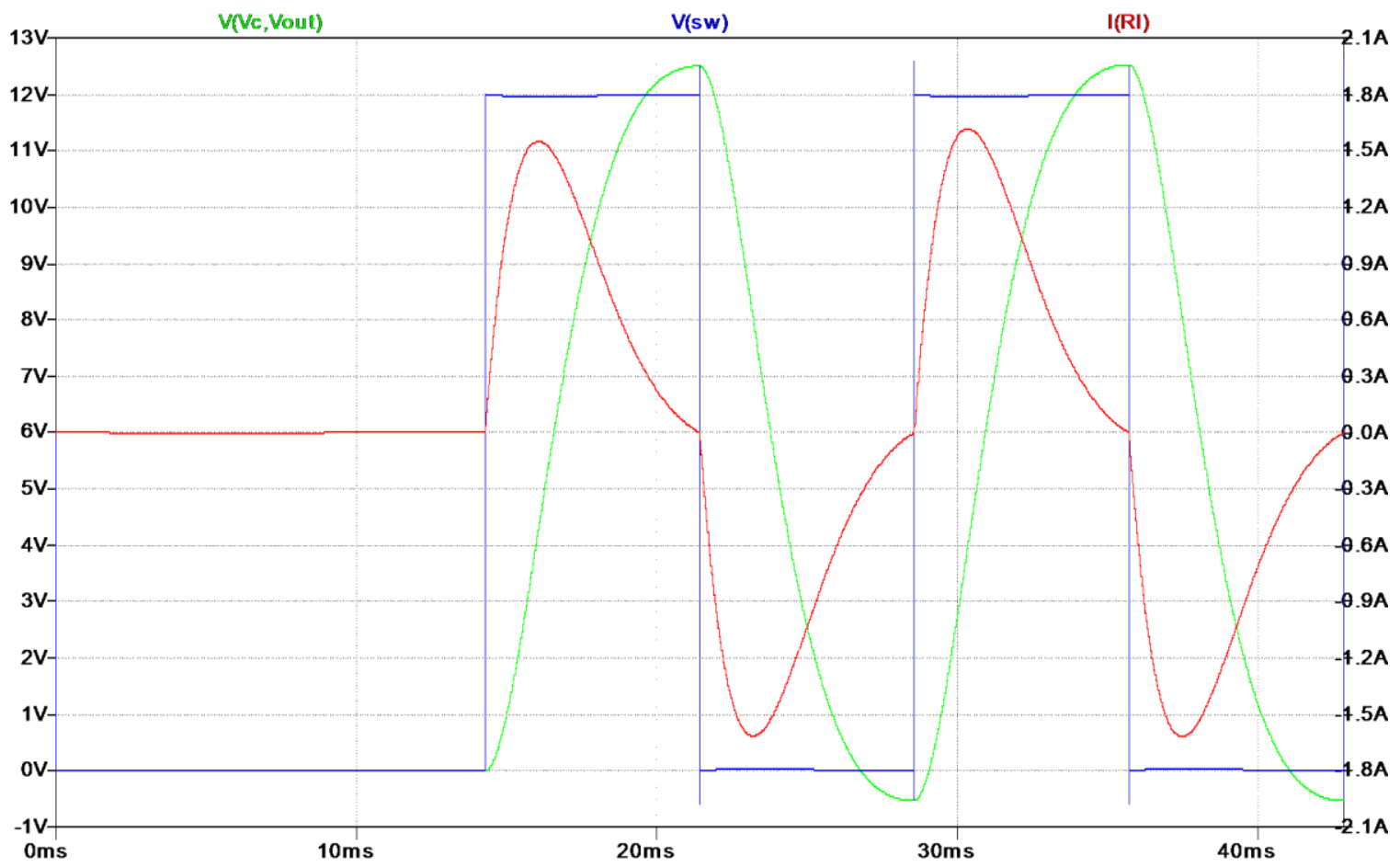

Figure 5-3: Capacitor voltage, switch node voltage, and output current demonstrating capacitor initial conditions per cycle

The proposed design method for optimum selection of inductor is performed for three frequencies which include $50 \mathrm{~Hz}, 60 \mathrm{~Hz}$, and $70 \mathrm{~Hz}$. For each frequency, input voltage, and total 
load resistance are kept constant. The input voltage is set to $12 \mathrm{~V}$ with a load resistance of $4.5 \Omega$, and parasitic resistances of $0.5 \Omega$; thus, a total path resistance of $5 \Omega$. The inductance is varied and compared to the RMS output current to evaluate an optimum operating point. Figure 5-4 to Figure 5-6 demonstrate simulation results of output current vs inductance, and the corresponding error between the steady state and calculated RMS output current is shown in Figure 5-7.

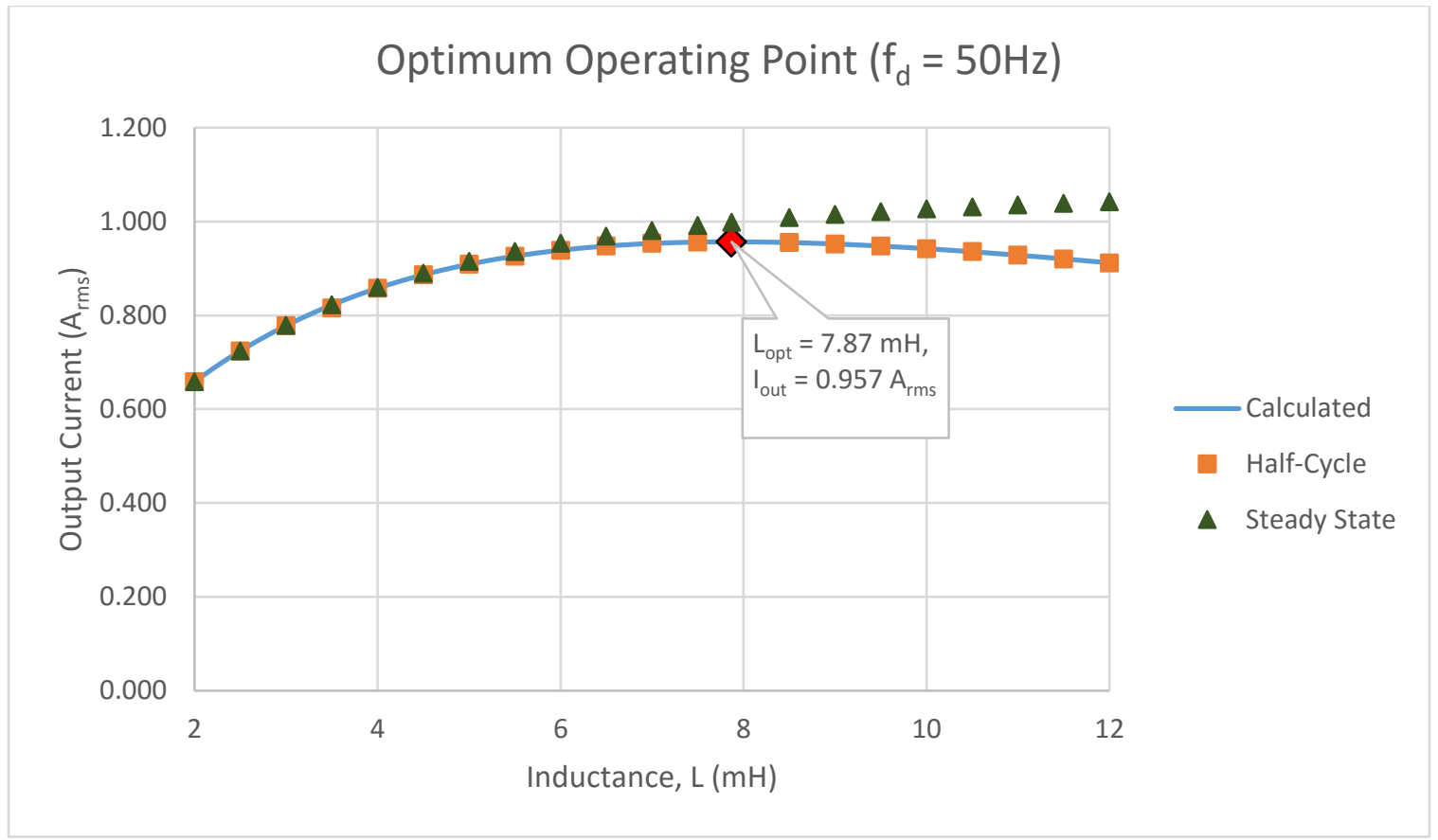

Figure 5-4: RMS output current vs inductance for $50 \mathrm{~Hz}$ operation 


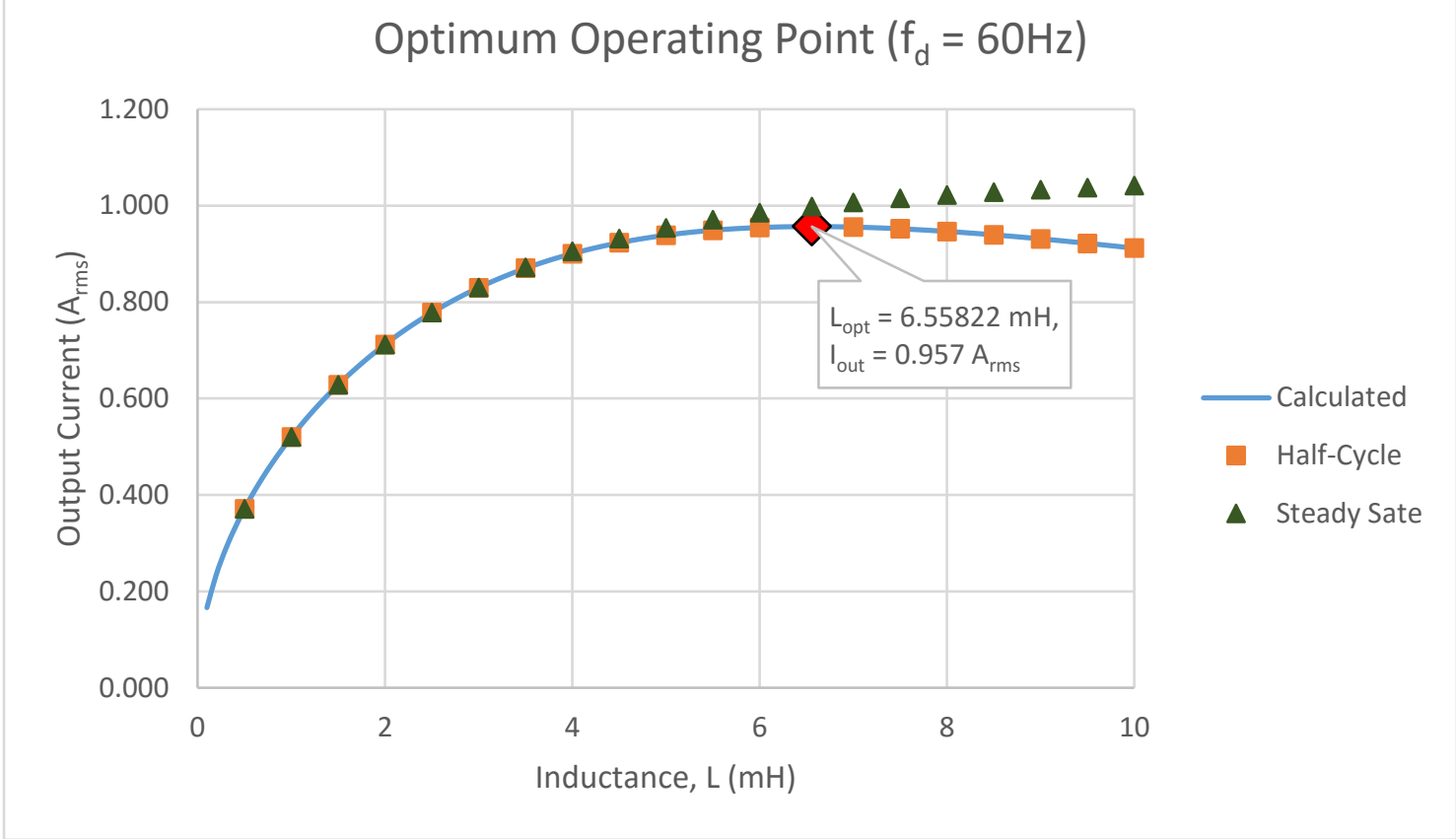

Figure 5-5: RMS output current vs inductance for $60 \mathrm{~Hz}$ operation

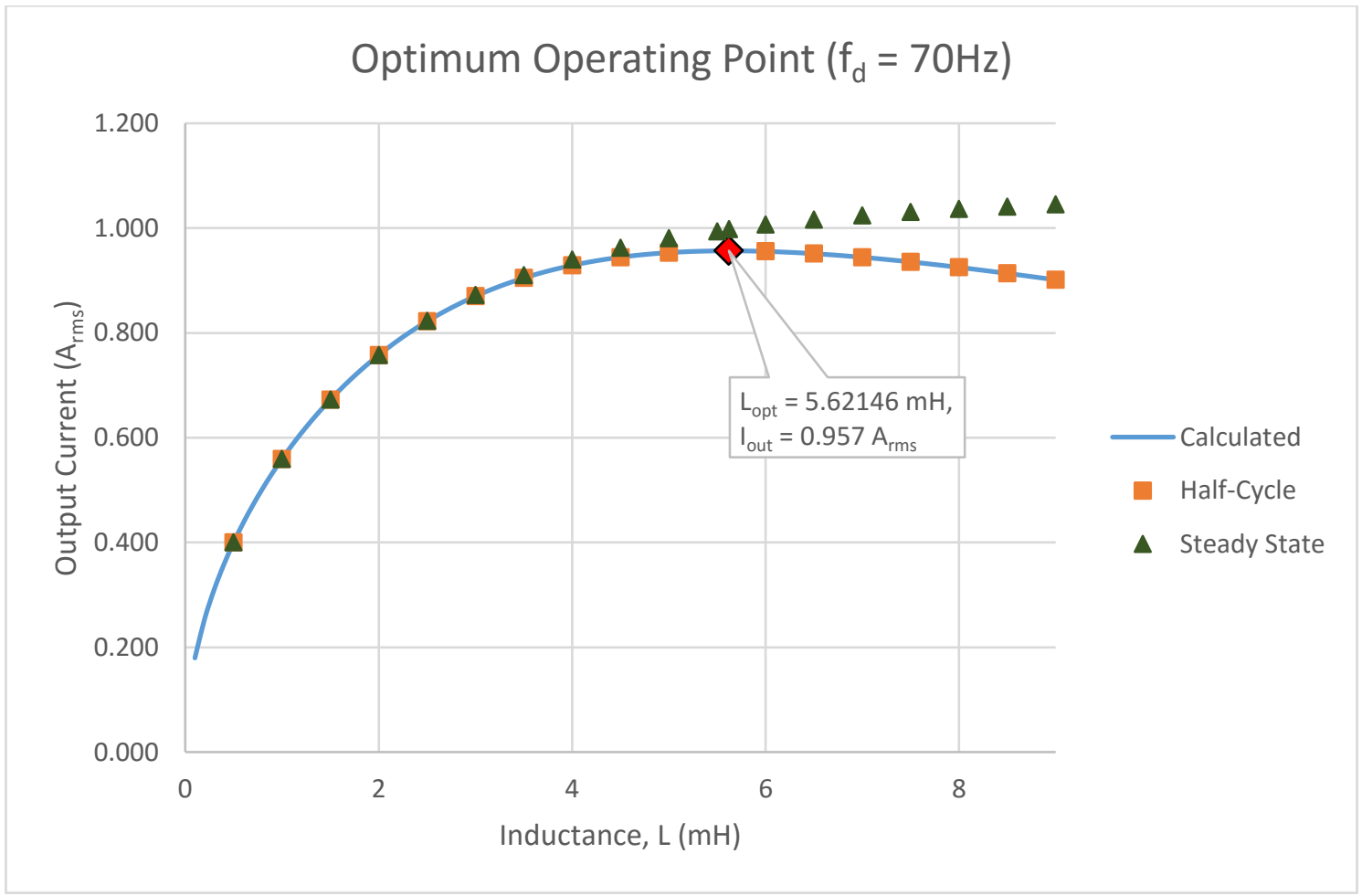

Figure 5-6: RMS output current vs inductance for $70 \mathrm{~Hz}$ operation 


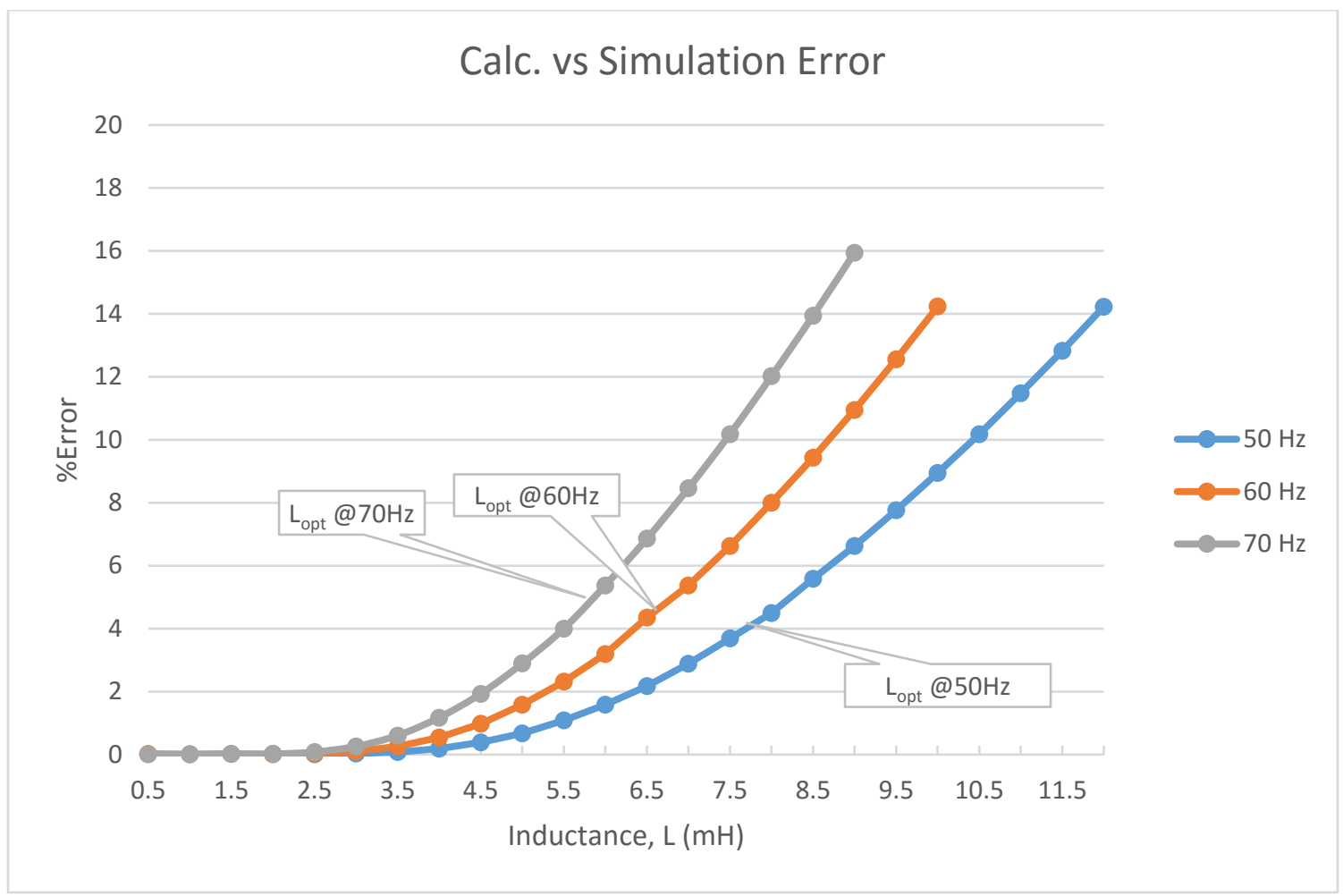

Figure 5-7: Percent error between calculated and steady state RMS output voltage

The results demonstrate a correspondence between the mathematical model and the first half-cycle of simulation. This result is expected as both the mathematical model and the first halfcycle of simulation disregard initial conditions. Figure 5-4 to Figure 5-6 demonstrate the simulated steady state output current increases as inductance increases; thus, disagreeing with the mathematical model. This is attributed to the absence of initial conditions in the mathematical model. For more inductance, more energy is stored in the inductor; therefore, more energy is transferred to the capacitor, which corresponds to greater influence of initial conditions. Figure 5-7 shows the error between the mathematical model and the steady state error. Note that the error remains below $5 \%$ for all three optimum inductor values; thus, the mathematical model is accurate to below $5 \%$ for the optimum inductor selection design method. Although a greater inductance may be selected to achieve a larger output RMS voltage, the benefit diminishes beyond the optimum operating point. Below the optimum inductance, a small change inductance results in a large change in output RMS current; however, beyond the optimum inductance a 
small change in inductance results in a small change in output RMS current. To demonstrate this behavior, the output current sensitivity to inductance is obtained using the discrete difference between data points of the steady state RMS output voltage.

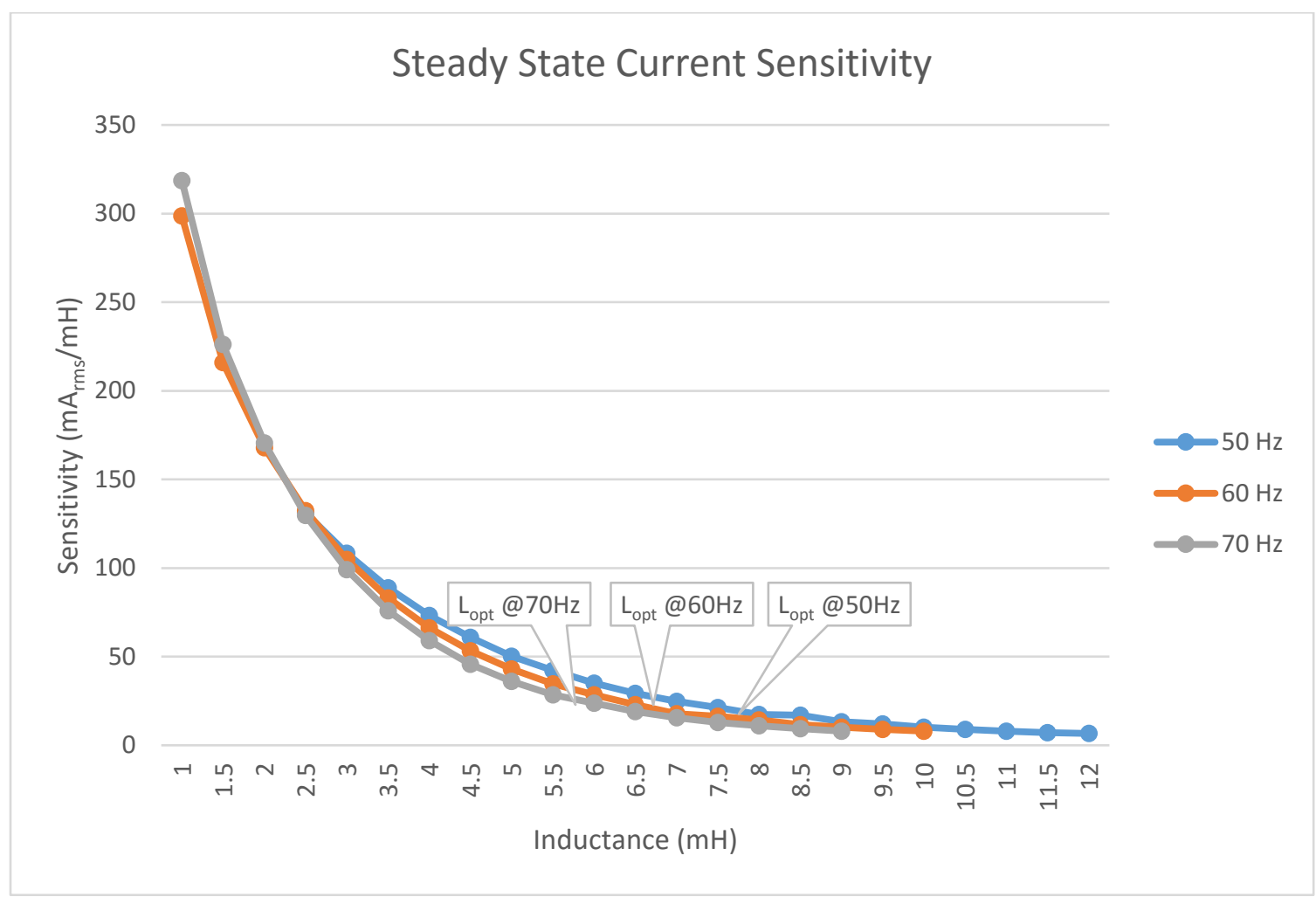

Figure 5-8: Output current sensitivity to inductor selection

Figure 5-8 demonstrates output current sensitivity to inductance. Beyond the optimum inductance, sensitivity drops below $50 \mathrm{~mA}_{\mathrm{rms}} / \mathrm{mH}$; thus, quantifying the effect where small changes in inductance beyond the optimum operating point result in a minimal change in output RMS voltage. The optimum inductor selection method is a practical design, since larger inductances require larger magnetic components, which increase system size and cost.

In addition to the simulations performed in this section, investigation of SLR inverter performance includes frequency stability, load and line regulation, total harmonic distortion and efficiency. The simulation models developed include $50 \mathrm{~Hz}, 60 \mathrm{~Hz}$, and $70 \mathrm{~Hz}$ designs. The 
parameters of these models are summarized in Table 5-1 to Table 5-3. These models are used in the following sections to investigate SLR inverter performance.

Table 5-1: Simulation parameters of 50Hz SLR inverter design

\begin{tabular}{|l|r|}
\hline $\mathbf{L}_{\text {opt }}(\mathbf{m H})$ & 7.87 \\
\hline $\mathbf{C}_{\text {opt }}(\boldsymbol{\mu} \mathbf{F})$ & 636.58 \\
\hline $\mathbf{R}_{\mathbf{L}} \mathbf{( \Omega )}$ & 4.50 \\
\hline $\mathbf{R}_{\text {DS(on) }}(\mathbf{m} \mathbf{\Omega})$ & 25.00 \\
\hline $\mathbf{R}_{\text {IND }}(\mathbf{m} \mathbf{\Omega})$ & 450.00 \\
\hline $\mathbf{R}_{\text {ESR }}(\mathbf{m} \mathbf{\Omega})$ & 25.00 \\
\hline $\mathbf{R}_{\text {PAR }}(\mathbf{\Omega})$ & 0.5 \\
\hline $\mathbf{R}_{\text {TOT }}(\mathbf{\Omega})$ & 5 \\
\hline $\mathbf{f}_{\mathbf{d}} \mathbf{( H z )}$ & 50 \\
\hline
\end{tabular}

Table 5-2:Simulation parameters of $60 \mathrm{~Hz}$ SLR inverter design

\begin{tabular}{|l|r|}
\hline $\mathbf{L}_{\text {opt }}(\mathbf{m H})$ & 6.56 \\
\hline $\mathbf{C}_{\text {opt }}(\boldsymbol{\mu} \mathbf{F})$ & 530.47 \\
\hline $\mathbf{R}_{\mathbf{L}} \mathbf{( \Omega )}$ & 4.50 \\
\hline $\mathbf{R}_{\text {DS(on) }}(\mathbf{m} \mathbf{\Omega})$ & 25.00 \\
\hline $\mathbf{R}_{\text {IND }}(\mathbf{m} \mathbf{\Omega})$ & 450.00 \\
\hline $\mathbf{R}_{\text {ESR }}(\mathbf{m} \mathbf{\Omega})$ & 25.00 \\
\hline $\mathbf{R}_{\text {PAR }}(\mathbf{\Omega})$ & 0.5 \\
\hline $\mathbf{R}_{\text {TOT }}(\mathbf{\Omega})$ & 5 \\
\hline $\mathbf{f}_{\text {d }}(\mathbf{H z})$ & 60 \\
\hline
\end{tabular}


Table 5-3: Simulation parameters of 70Hz SLR inverter design

\begin{tabular}{|l|r|}
\hline $\mathbf{L}_{\text {opt }}(\mathbf{m H})$ & 5.62 \\
\hline $\mathbf{C}_{\text {opt }}(\boldsymbol{\mu F})$ & 454.70 \\
\hline $\mathbf{R}_{\mathbf{L}}(\mathbf{\Omega})$ & 4.50 \\
\hline $\mathbf{R}_{\text {DS(on) }}(\mathbf{m} \mathbf{\Omega})$ & 25.00 \\
\hline $\mathbf{R}_{\text {IND }}(\mathbf{m} \mathbf{\Omega})$ & 450.00 \\
\hline $\mathbf{R}_{\text {ESR }}(\mathbf{m} \mathbf{\Omega})$ & 25.00 \\
\hline $\mathbf{R}_{\text {PAR }}(\mathbf{\Omega})$ & 0.50 \\
\hline $\mathbf{R}_{\text {TOT }}(\mathbf{\Omega})$ & 5.00 \\
\hline $\mathbf{f}_{\mathbf{d}}(\mathbf{H z})$ & 70.00 \\
\hline
\end{tabular}

\subsection{Hardware Prototype}

The hardware prototype is measured in lab to verify design requirements. A variable power resistor is connected to the output and adjusted to $4.7 \Omega$. MPJA-9313 power supply is connected to the DC input and set to $18.7 \mathrm{~V}_{\mathrm{DC}}$. An auxiliary GPR-6060D power supply is set to $12 \mathrm{~V}$ and connected to the control circuitry. The frequency regulating potentiometer is manually adjusted to achieve an output frequency as close to $70 \mathrm{~Hz}$ as possible. The voltage across the load is observed using LeCroy HDO-4104 oscilloscope. The laboratory setup for testing the SLR inverter is demonstrated in Figure 5-9. The output voltage waveform is demonstrated in Figure 5-10. 


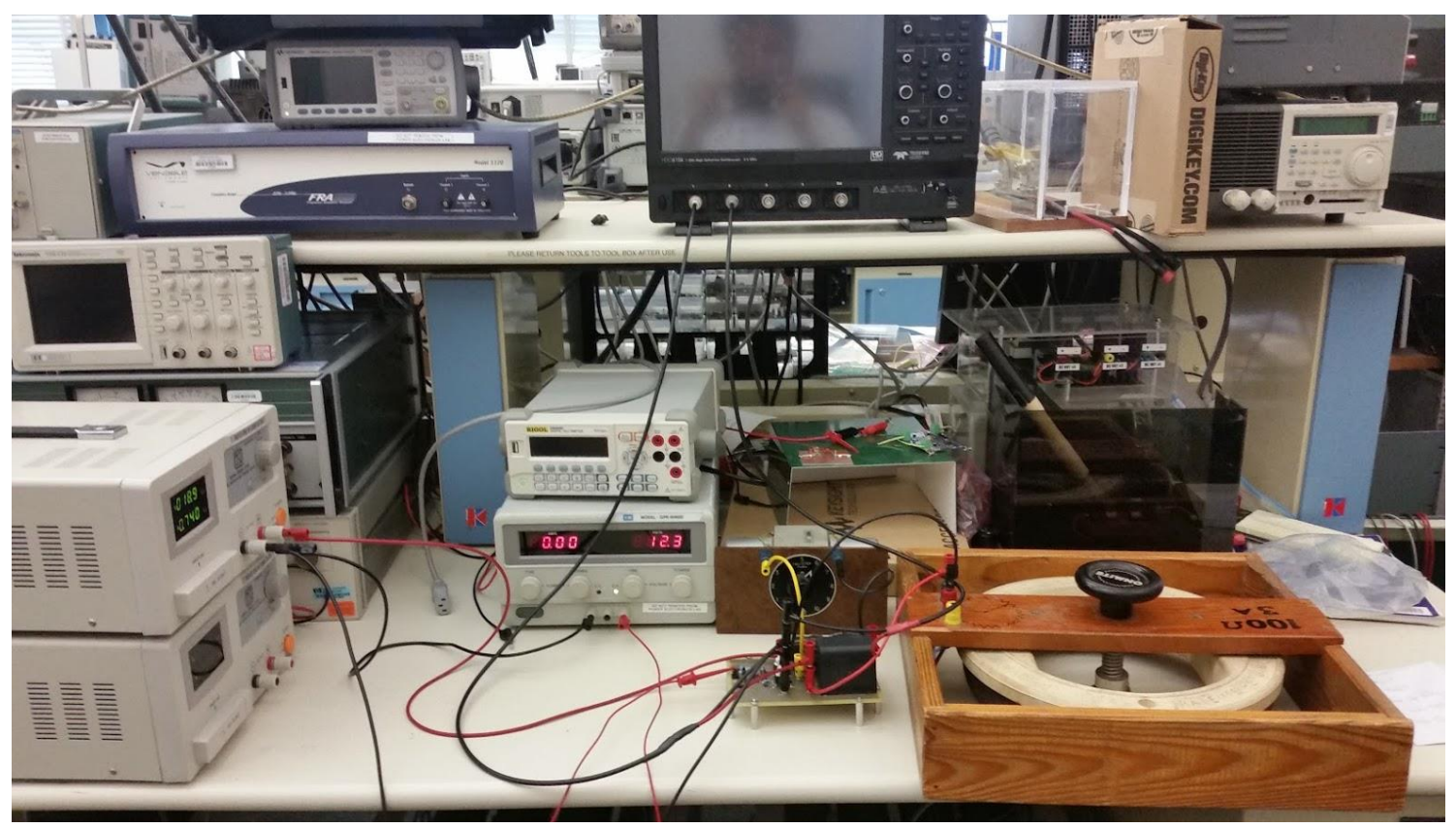

Figure 5-9: SLR inverter prototype laboratory setup

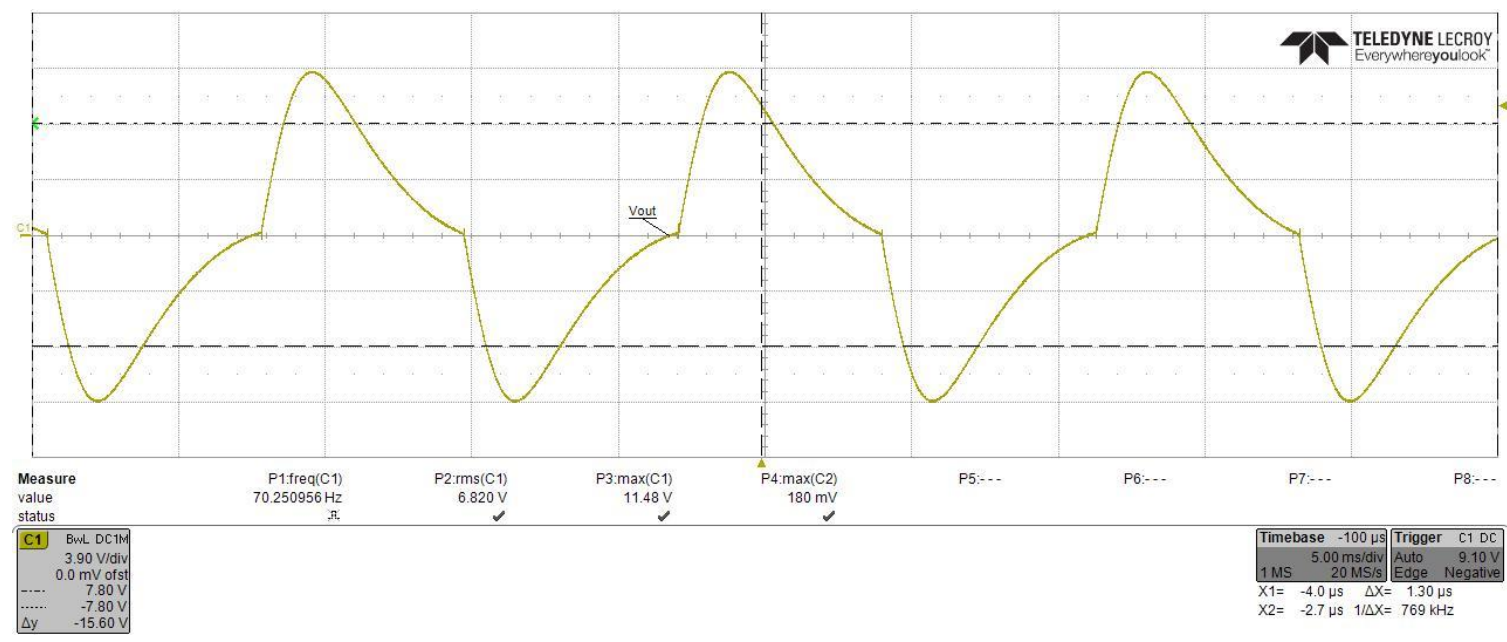

Figure 5-10: SLR inverter prototype output voltage

The voltage waveform observed in Figure 5-10 demonstrates a correlation to simulation. The output resembles the damped sine wave predicted by the mathematical model. The frequency of the output is measured to $70.3 \mathrm{~Hz}$. This inaccuracy is attributed to switching inaccuracies produced by the controller and turn-on/turn-off times of the MOSFETs. Output RMS voltage is measured to be $6.82 \mathrm{~V}_{\mathrm{RMS}}$, resulting in $9.9 \mathrm{~W}$ of power consumption, which is near the design 
output power of $10 \mathrm{~W}$. This error is attributed to inductor and capacitor inaccuracies. Additionally, the parasitic resistances were assumed to total $0.5 \Omega$; however, measurement demonstrates a total of $0.2 \Omega$, which also contributes to output power error. Nevertheless, these inaccuracies result in $1 \%$ error, which demonstrates the accuracy of the design equations. Summary of the design vs. measured parameters are shown in Table 5-4.

Table 5-4: SLR inverter prototype design vs. measurement

\begin{tabular}{|c|c|c|}
\hline & Design & Measured \\
\hline Frequency $(\mathrm{Hz})$ & 70.00 & 70.30 \\
\hline Input Voltage ( $\left.\mathbf{V}_{\text {DC }}\right)$ & 18.70 & 18.7 \\
\hline $\mathbf{L}_{\text {opt }}(\mathrm{mH})$ & 5.62 & 5.76 \\
\hline $\mathrm{C}_{\text {opt }}(\mu \mathrm{F})$ & 454.70 & 458.38 \\
\hline $\mathbf{R}_{\text {load}}(\mathbf{\Omega})$ & 4.50 & 4.70 \\
\hline $\mathbf{R}_{\mathrm{par}}(\mathbf{\Omega})$ & 0.50 & 0.20 \\
\hline $\mathbf{R}_{\text {total }}(\mathbf{\Omega})$ & 5.00 & 4.9 \\
\hline Output Power (W) & 10.00 & 9.9 \\
\hline
\end{tabular}

Further analysis of the SLR inverter is performed to understand operating characteristics. These parameters include efficiency, ZVS, initial capacitor voltage, and total harmonic distortion. Rigol DM3058E multi-meter measures a $1.03 \mathrm{~A}_{\mathrm{RMS}}$ input current to the SLR inverter; thus, a total input power of $19.26 \mathrm{~W}$ and an efficiency of $51.4 \%$. A higher efficiency is expected due to the measured parasitic resistances; however, it is important to note that these parasitics were measured with minimal inductor current. For the case of the loaded inductor, greater current through the inductor results in greater hysteresis and eddy current losses. To obtain more accurate measurements of inductor parasitics, measurement of the parasitics should be obtained at the desired load. Based on equation (3-26), total parasitic resistance is calculated to be $4.44 \Omega$; 
however, these loses do not compromise the output power design equation, since the influence of total path resistance is minimal in equation (3-17).

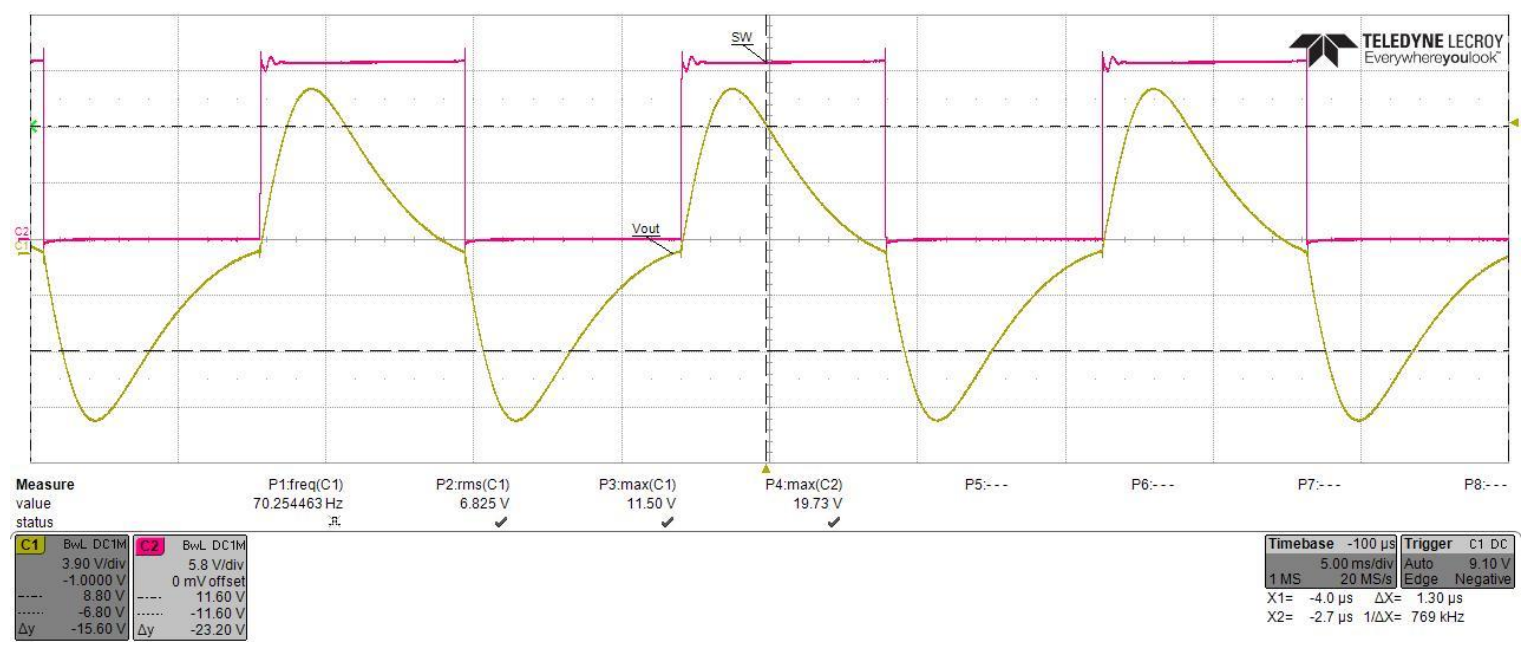

Figure 5-11: SLR inverter prototype switch node (SW) and output voltage (Vout)

Figure 5-11 demonstrates output voltage and switch node. Output current is proportional to output voltage, since output voltage is measured across a resistive load. Switching occurs when voltage/current is near zero; thus, ZVS is achieved resulting in minimized switching losses.

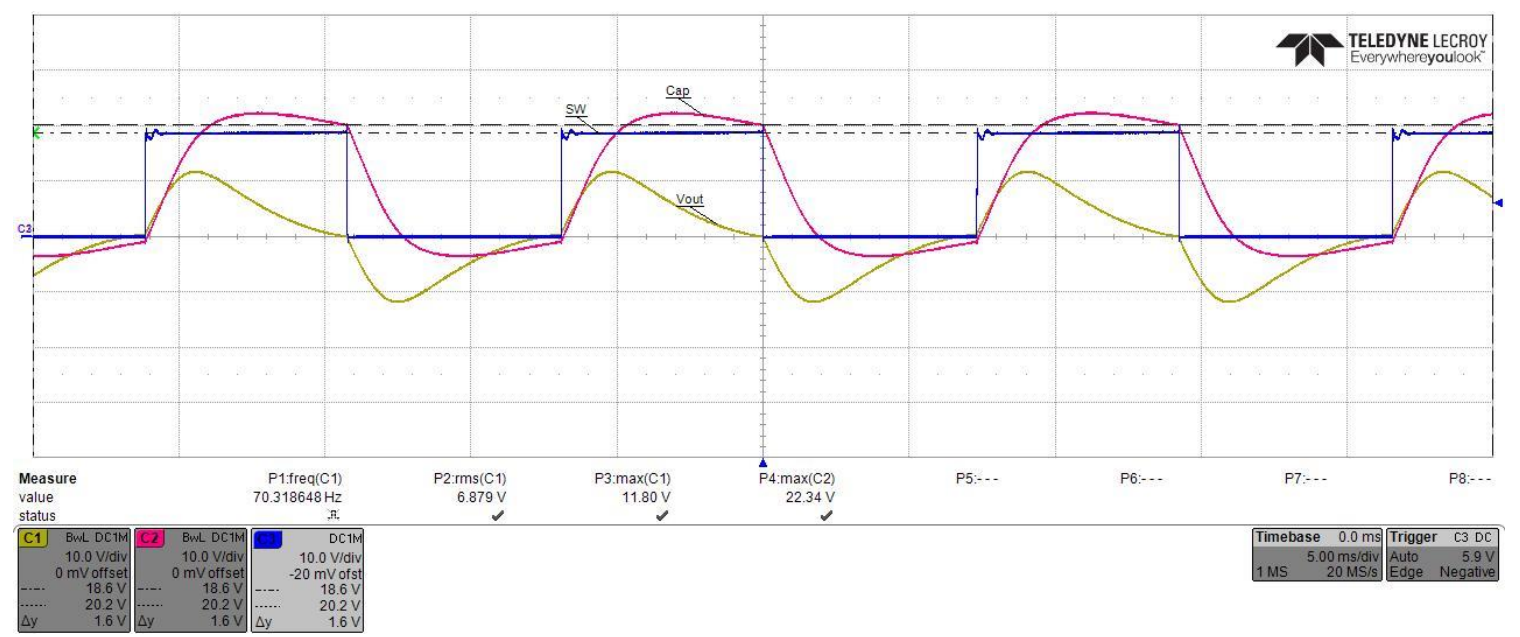

Figure 5-12: SLR inverter prototype switch node (SW), and capacitor voltage (Vcap)

Figure 5-12 demonstrates the relationship between the switch node and capacitor voltage. The mathematical model assumes the voltage across the capacitor is $0 \mathrm{~V}$ before a positive 
transition; however, a 1.6V deviation exists contributing to the error between design and measurement. Similarly, the voltage across the capacitor is assumed to be equivalent to the input voltage before a negative transition; nevertheless, a 1.6V deviation contributes to the error between design and measurement.

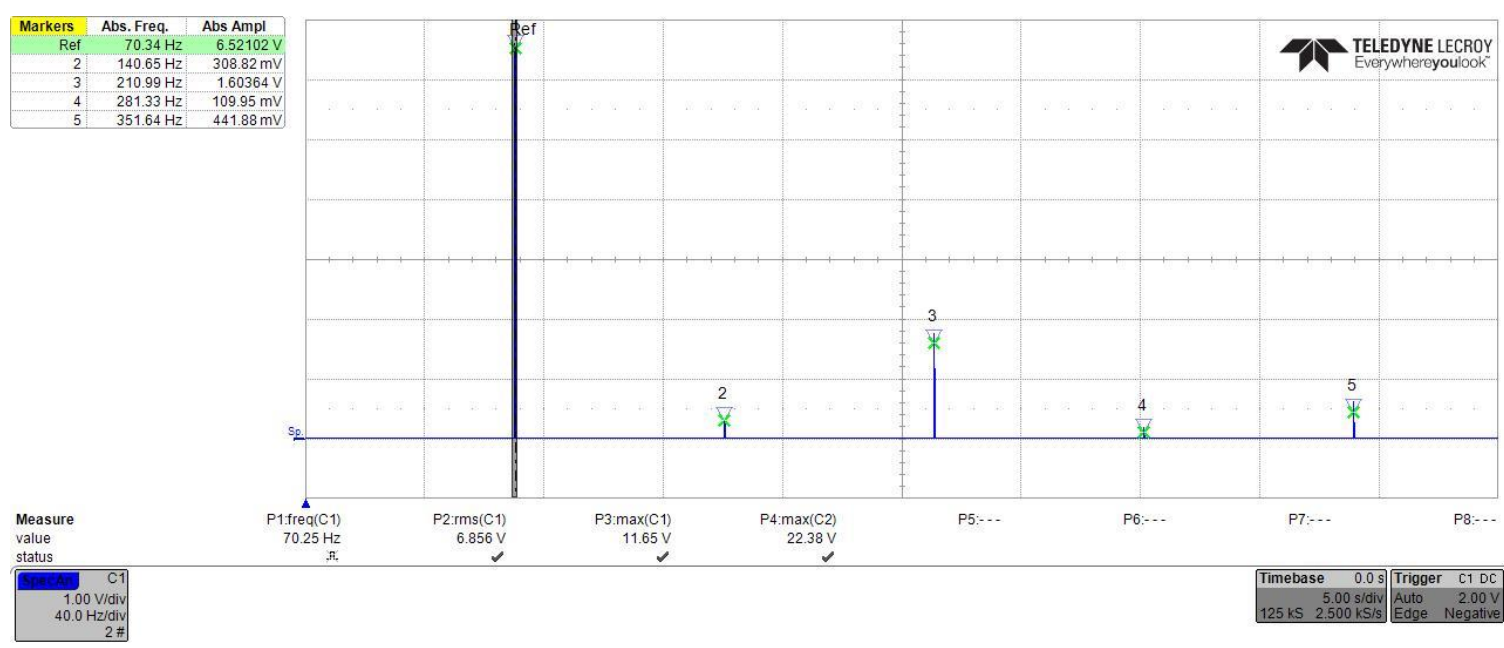

Figure 5-13: SLR inverter prototype output voltage THD

THD is measured using the LeCroy HDO-4104 spectrum feature, where Fast Fourier Transform (FFT) is performed on the output voltage. The FFT is taken for a total of $10 \mathrm{~s}$ of data and a flat top windowing function is used to evaluate data. The result of the FFT is demonstrated in Figure 5-13. The THD is calculated for the first 5 harmonics of the fundamental frequency. A table for the harmonics, frequency, and RMS voltage is shown at top right of Figure 5-13. Based on these measured harmonics, $30.7 \%$ THD is determined for the $70 \mathrm{~Hz}$ SLR inverter prototype. The same procedure is repeated for all THD measurements in this chapter.

To examine the effects of variable inductance, the fixed design inductance is replaced with a General Radio 940-E variable inductor. The inductance is varied from $1 \mathrm{mH}$ to $10 \mathrm{mH}$ in $1 \mathrm{mH}$ steps to measure frequency stability, THD, current regulation, and efficiency. The inductance and parasitic resistance is measured for each setting of the variable inductor, shown in 
Table 5-5. For each inductance value, a corresponding load resistance is set via a variable resistor, so as to achieve $70 \mathrm{~Hz}$ frequency stability. Each setting is measured for frequency stability, current regulation, THD, and efficiency. The input voltage is set to $12 \mathrm{~V}_{\mathrm{DC}}$ to compare simulation to hardware. Results are presented in the following sections.

Table 5-5: General Radio 940-E variable inductor measured parameters

\begin{tabular}{|r|r|}
\hline $\mathbf{L}(\mathbf{m H})$ & \multicolumn{1}{|c|}{$\mathbf{R}_{\mathbf{S}}(\mathbf{m} \boldsymbol{\Omega})$} \\
\hline 0.99 & 82.03 \\
\hline 2.01 & 158.60 \\
\hline 2.97 & 174.02 \\
\hline 3.99 & 280.14 \\
\hline 4.99 & 282.72 \\
\hline 5.98 & 347.25 \\
\hline 6.99 & 409.82 \\
\hline 7.96 & 445.28 \\
\hline 8.98 & 508.12 \\
\hline 9.97 & 604.46 \\
\hline
\end{tabular}

\subsection{Resonant Frequency Stability}

The dominant issue with resonant inverters is the sinusoid resonant frequency, which is dependent on load resistance. Ideal frequency of resonance, $\omega_{0}$, is established by the series inductance and capacitance of the SLR inverter, given by equation (3-5). The sinusoid ideal frequency of resonance occurs when resistive elements are absent in the SLR inverter. In practical circuits, a finite resistance is always present causing the ideal frequency of resonance to diminish. The diminished frequency is referred to as the damped frequency, $\omega_{\mathrm{d}}$, and is given by equation (3- 
6). To further characterize the load dependent nature of the SLR inverter, the expected frequency of equation (3-6) is compared to the simulation model of Figure 5-1.

Simulation of resonant frequency dependence on load is performed for the three previously designed simulation models. The parameters of these models are demonstrated in Table 5-1 to Table 5-3. For each model, the chosen optimum inductor and capacitor values remain constant while the load is varied from 0 to $4.5 \Omega$ in steps of $0.5 \Omega$. The resonant frequency is measured in simulation for each load setting. The frequency is measured by applying a step input to the SLR inverter and measuring the time difference between the zero-crossings of the output voltage. This time difference corresponds to a half-period and is plotted vs the theoretically calculated frequency. The results of the simulation are shown in Figure 5-14-Figure 5-16. Furthermore, Figure 5-1 demonstrates hardware results for the SLR inverter prototype. The simulation results validate the mathematical model for frequency given by equation (3-6). An error is observed for the prototype frequency stability, which is attributed to component tolerances.

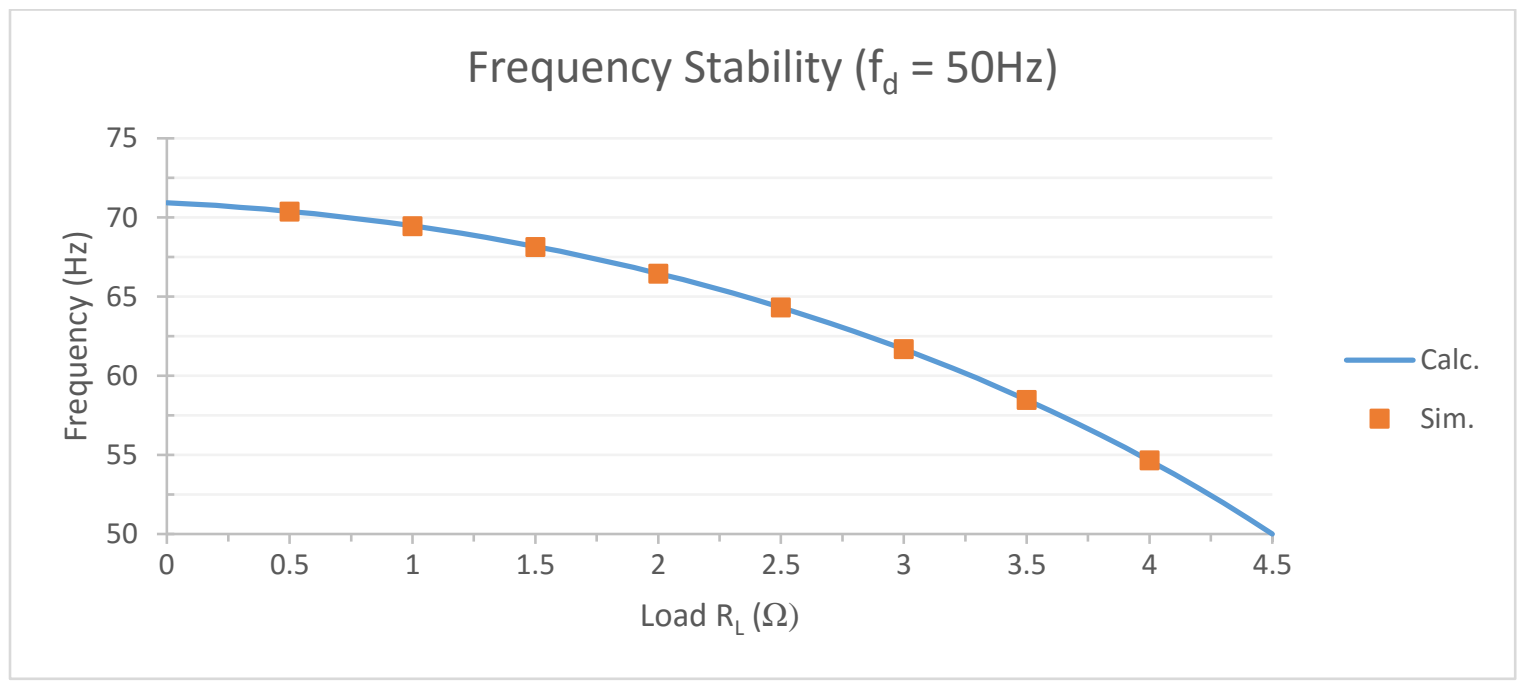

Figure 5-14: Resonant frequency vs. load for $50 \mathrm{~Hz}$ design 


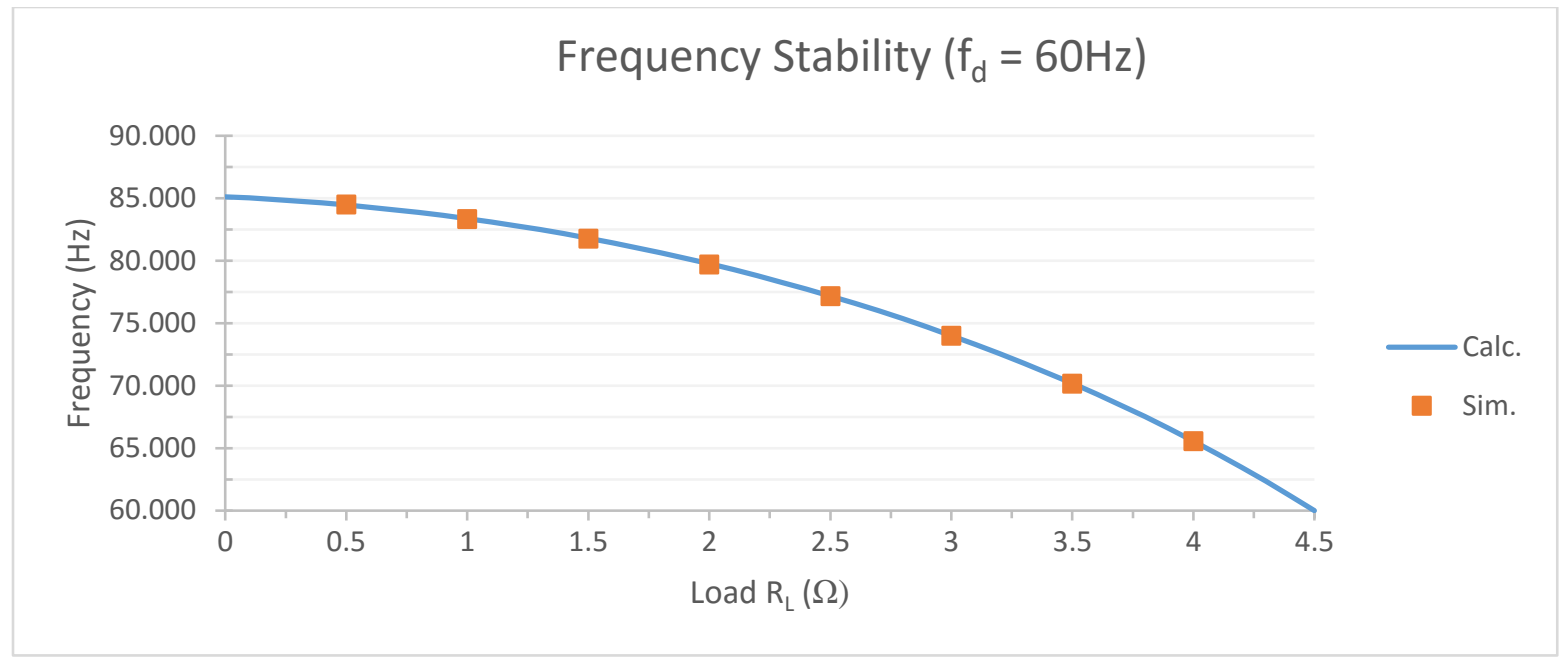

Figure 5-15: Resonant frequency vs. load for $60 \mathrm{~Hz}$ design

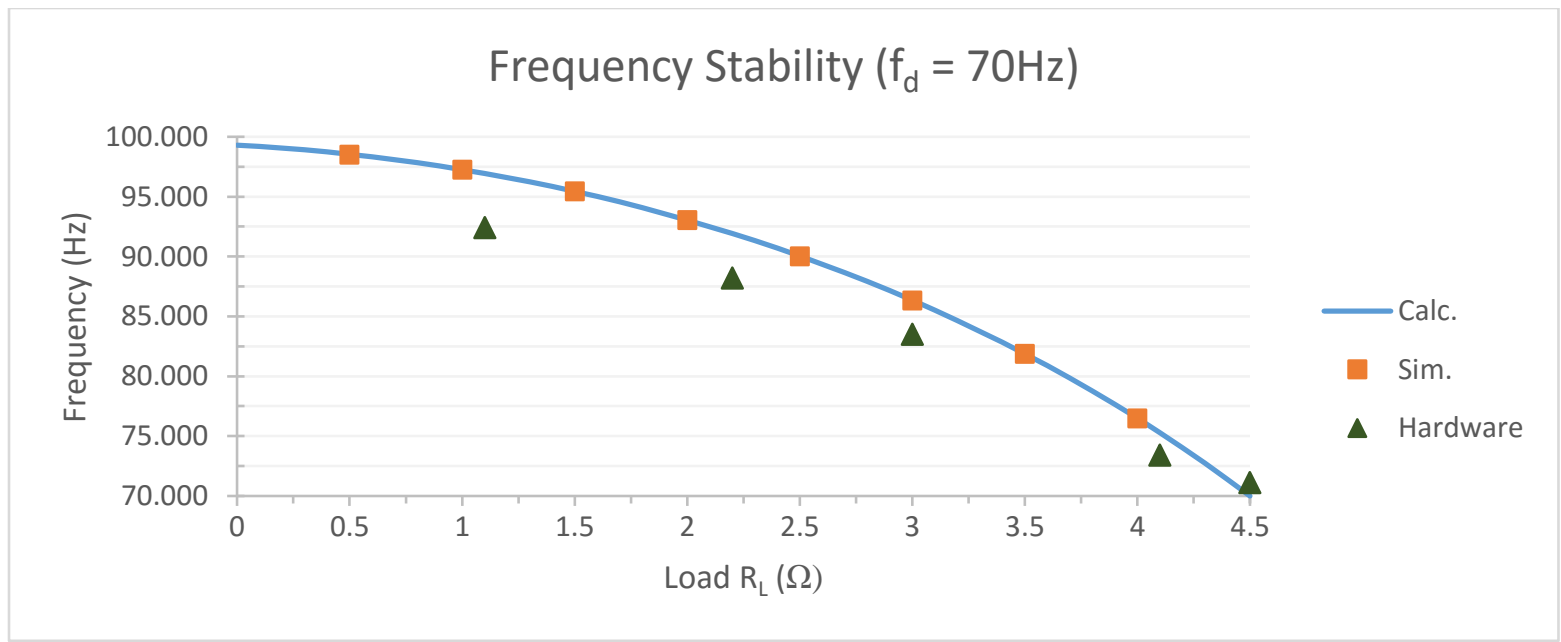

Figure 5-16: Resonant frequency vs. load for $70 \mathrm{~Hz}$ design

Previously discussed in the literature, [25][26], demonstrate a promise for a low frequency electronically tunable inductor. An electronically tunable inductor may be the solution to frequency stability in resonant inverters; thus, it is important to characterize the necessary inductance range to maintain stability. To determine the inductance range, inductance of the SLR inverter is varied in accordance with the load to maintain a stable frequency. The capacitor is kept 
constant, and the load is varied from 0 to $4.5 \Omega$. For each load, a corresponding inductance is calculated using equation (3-6) and the results are shown in Figure 5-17.

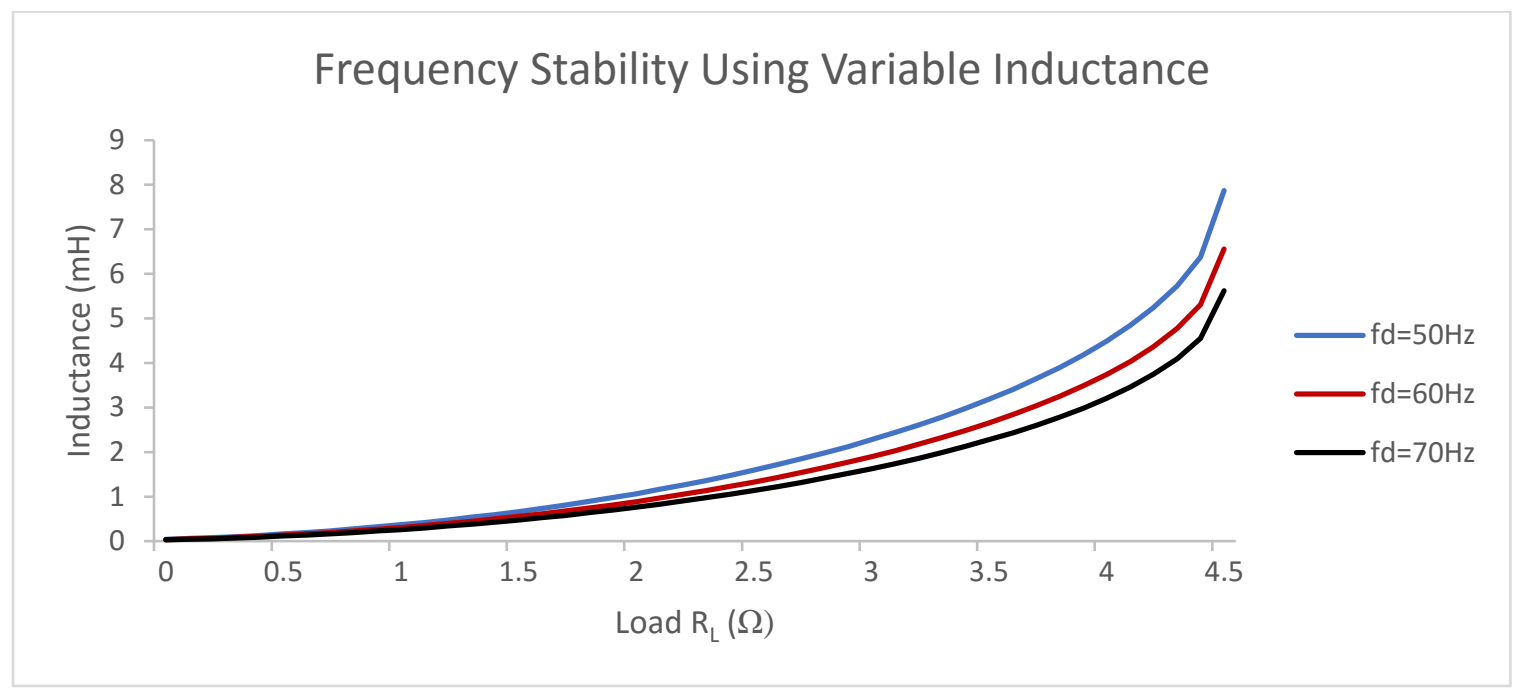

Figure 5-17: Inductance vs. load for constant frequency

The inductance range required to maintain stability for the entire load range is far beyond the range of the electronically tunable inductor [25]. The inductance range of the tunable inductor is $L_{\max } / L_{\min }=370 \%$ as specified in [25]; thus, a load range from $2.9 \Omega$ to $4.5 \Omega$ is achievable, as shown by the data obtained from Figure 5-17. It is interesting to note that frequency stability cannot be maintained for load resistances above the optimum load. This is evident from equation (3-6), since a solution for the required inductance cannot be determined. A mathematical justification using equation (3-6) is shown below to understand the limits of the required inductance for a given load when capacitance is kept constant.

$$
\begin{gathered}
\omega_{d}=\sqrt{\frac{1}{L C}-\left(\frac{R_{\text {total }}}{2 L}\right)^{2}} \\
\omega_{d}^{2}+\left(\frac{R_{\text {total }}}{2 L}\right)^{2}-\frac{1}{L C}=0
\end{gathered}
$$




$$
L^{2} \omega_{d}^{2}-\frac{L}{C}+\frac{R_{\text {total }}^{2}}{4}=0
$$

The required inductance vs. load resistance results in a quadratic equation. The load resistance term lies within $R_{\text {total }}$, which is the total series resistance of the circuit. To determine a solution to the quadratic equation, the quadratic formula is used, as shown below.

$$
L=\frac{\frac{1}{C} \pm \sqrt{\frac{1}{C^{2}}-4\left(\omega_{d}^{2}\right)\left(\frac{R_{\text {total }}{ }^{2}}{4}\right)}}{2 \omega_{d}^{2}}
$$

For a real solution to exist the square root term must always be greater than zero. Thus the limits of the load range for frequency stability may be determined by assuring this term is greater than or equal to 0 .

$$
\begin{gathered}
\frac{1}{C^{2}}-\omega_{d}^{2} R_{\text {total }}^{2} \geq 0 \\
\frac{1}{C^{2}} \geq \omega_{d}^{2} R_{\text {total }}^{2} \\
\sqrt{\frac{1}{\omega_{d}^{2} C^{2}}} \geq \sqrt{R_{\text {total }}^{2}} \\
\frac{1}{\omega_{d} C} \geq R_{\text {total }} \\
X_{c} \geq R_{\text {total }}
\end{gathered}
$$


The limiting factor of the load range is determined to be the reactance of the capacitor where $X_{c}=\frac{1}{\omega_{d} C}$. These results show that it is not useful to vary the inductance to maintain stability, since the load range is limited to resistances below the optimum design value. Alternately, to achieve stability the capacitance may be varied as the load changes. The capacitance required to maintain low frequency stability for the $50-70 \mathrm{~Hz}$ frequency range is on the order $10^{-4}$ Farads. Though electronically tunable capacitors exist, they are limited to the RF frequency range and thus frequency stability cannot be maintained with current technology.

Although a mathematical solution cannot be determined for the frequency, forced switching may accomplish frequency stability, but at the cost of switching losses. When switching losses must be accounted, the mathematical model fails, thus a new set of equations, which include switching losses must be developed. This effect is demonstrated in the hardware results shown in Figure 5-18. The results demonstrate a nose curve for the inductance vs. load, which verifies the condition for resonance predicted by equation (5-1). Beyond the optimum inductance (i.e. $6.56 \mathrm{mH}$ ), the load resistance must be diminished to maintain frequency stability; thus, frequency regulation for load resistances beyond the optimum operating load cannot be achieved without compromising switching losses. Additionally, required inductance to maintain frequency stability deviates from the calculated and simulated values. This is attributed to the increase in inductor parasitics as a function of inductance. Both calculation and simulation assume these parasitics remain constant; however, in practice greater parasitic resistances are expected for larger inductances, since more windings are required. This effect is demonstrated in the measured series resistance of the variable inductor shown in Table 5-5. 


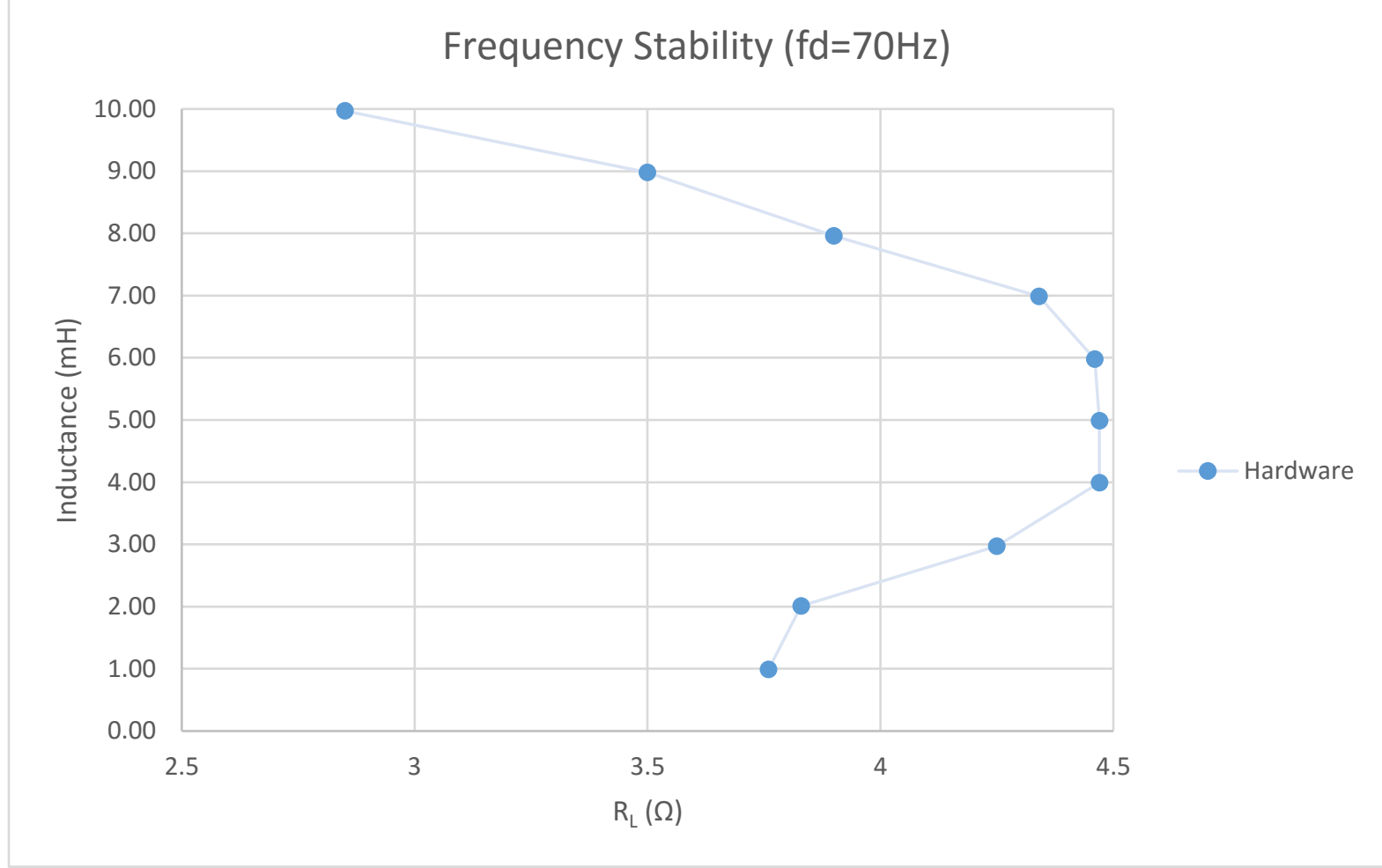

Figure 5-18: Frequency stability using variable inductance hardware results

\subsection{Load and Line Regulation}

Equation (3-16) reveals a linear relationship between output RMS current and input voltage. This allows for output power regulation of the SLR inverter by increasing or decreasing input voltage. The mathematical model used to develop equation (3-16) disregards initial conditions, thus the linear relationship between output current and input voltage must be verified via simulation. The linear relationship between output current and input voltage is verified in simulation via LTspice to determine the accuracy of the equation (3-16).

Load and line regulation is simulated using the three SLR inverter models and their parameters are shown in Table 5-1 to Table 5-3. To demonstrate line regulation, the load is varied from $0 \Omega$ to $4.5 \Omega$ while frequency is kept constant. Frequency stability is achieved by adjusting inductance while optimum capacitance remains constant. To simulate line regulation, three different input voltages are used to plot line regulation, which include $12 \mathrm{~V}, 24 \mathrm{~V}$, and $48 \mathrm{~V}$. The 
results of load and line regulation simulations for the $50 \mathrm{~Hz}, 60 \mathrm{~Hz}$, and $70 \mathrm{~Hz}$ inverter models is shown in Figure 5-19 to Figure 5-21, respectively.

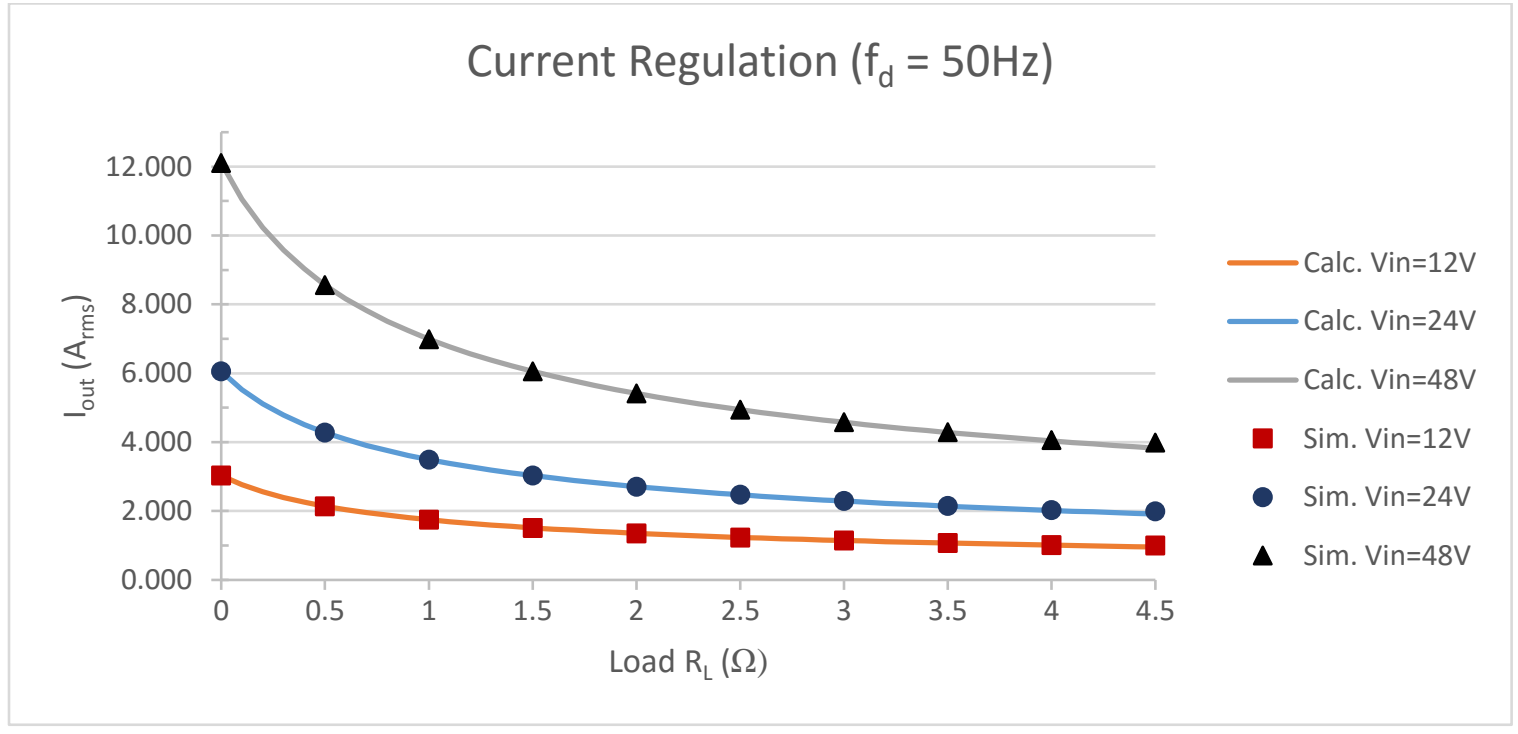

Figure 5-19: Load and line regulation for $50 \mathrm{~Hz}$ design using variable inductance frequency stability

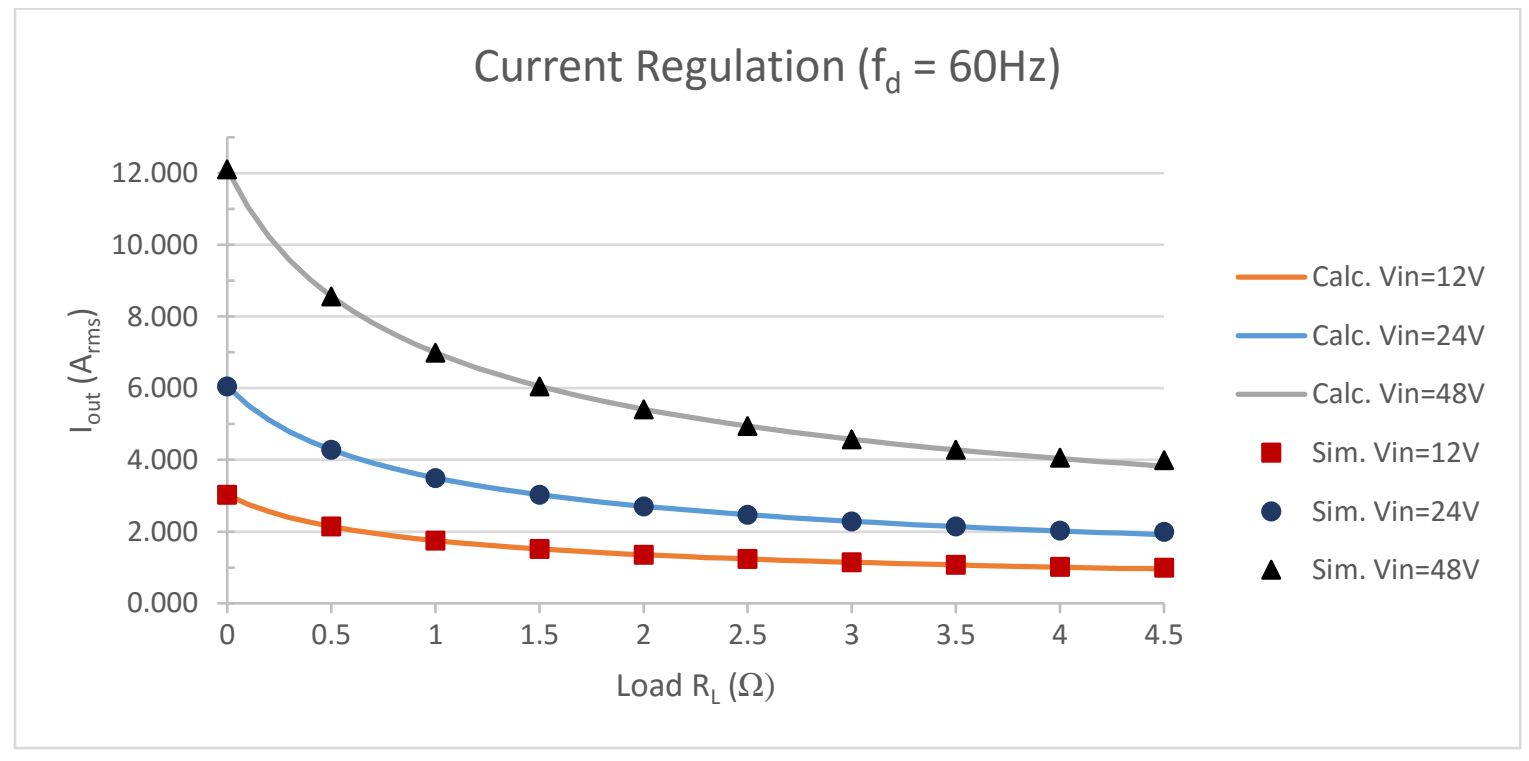

Figure 5-20: Load and line regulation for $60 \mathrm{~Hz}$ design using variable inductance frequency stability 


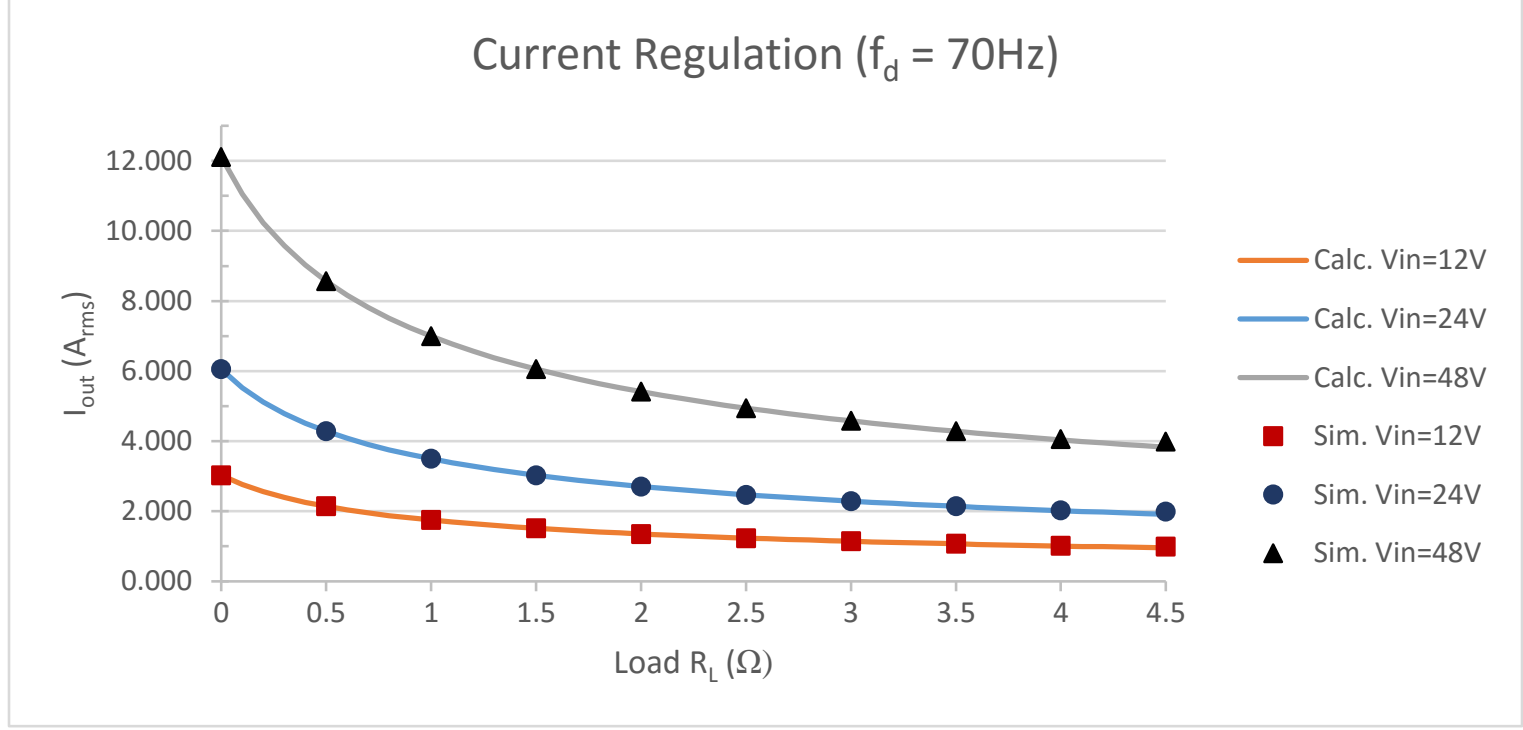

Figure 5-21: Load and line regulation for $70 \mathrm{~Hz}$ design using variable inductance frequency stability

The results of load and line regulation from Figure 5-19 to Figure 5-21 demonstrate agreement between the expected and simulation values. Greater inaccuracies are observed near the optimum load of $4.5 \Omega$, which is expected by the results shown in Figure 5-7 demonstrating the error between theoretical and steady state values of the output current. This confirms the linear relationship between the input voltage and output current; thus, voltage regulation at the input of the SLR inverter may be used to achieve output current regulation. Note that load resistance near the optimum load value of $4.5 \Omega$ requires less regulation. Specifically, for a small change in load resistance the output current remains fairly constant for load resistances within a range close to $4.5 \Omega$. If frequency stability is maintained via variable inductance, the load resistance is limited by the reactance of the capacitor as shown by equation (5-1). This limits the regulation performance of the SLR inverter.

In addition, it is important to note that in the event of a short-circuit condition (i.e. $\mathrm{R}_{\mathrm{L}}=0 \Omega$ ) the output current is clamped to a finite value. This provides the circuit with inherent short circuit protection as predicted by equation (3-16), since the output current is not proportional to the load. 
The three inverter models exhibit nearly identical load and line regulation. This effect demonstrates the output current of the SLR inverter is insensitive to the operational frequency between the range of $50 \mathrm{~Hz}$ to $70 \mathrm{~Hz}$. This is expected for lower frequencies; however, as the operational frequency of the inverter increases the developed inverter model must consider the parasitic inductance and capacitances of all components.

Hardware results shown in Figure 5-22 demonstrate a maximum output current deviation of $0.55 \mathrm{~A}_{\text {rms }}$ for a load range between $2.85 \Omega$ and $4.47 \Omega$. Note that regulation cannot be achieved beyond the optimum load of $4.5 \Omega$. Once again, this is attributed to limitations imposed by equation (5-1). For inductances below the optimum design inductance the maximum error between simulation and hardware is $2.7 \%$. This corresponds to the lower portion of the nose curve in Figure 5-22: Current regulation using variable inductance hardware results.

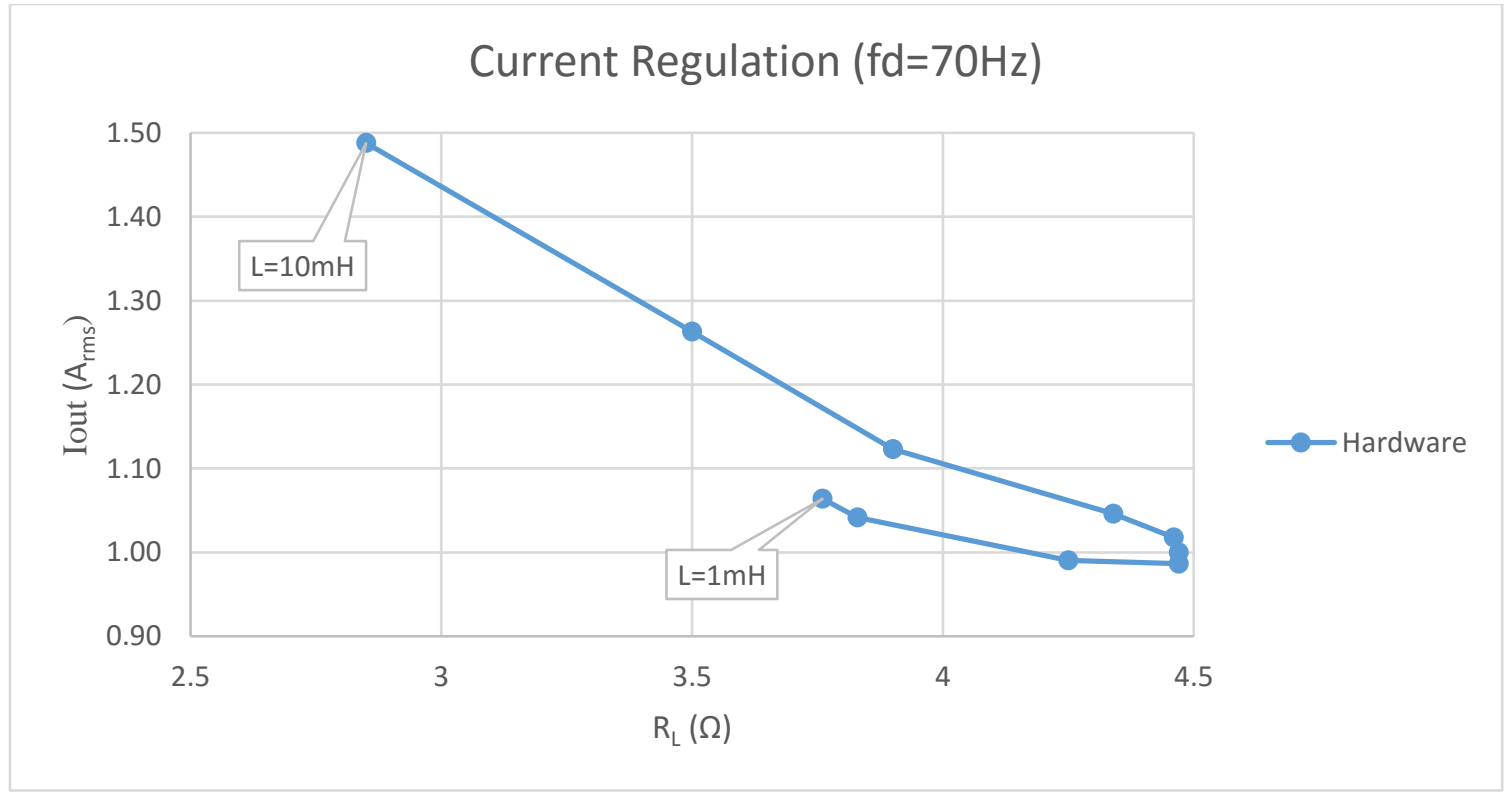

Figure 5-22: Current regulation using variable inductance hardware results 


\subsection{Total Harmonic Distortion}

The series capacitor and inductor in the SLR inverter form a band pass filter, thus attenuating frequencies below and beyond the resonant frequency. A square wave input present at the switch node presents odd order harmonics of the switching frequency to the series capacitor and inductor. These harmonics diminish the power quality of the output; thus, measuring the harmonics produced is detrimental to system performance. Total harmonic distortion (THD) is defined as the ratio between the total RMS of harmonic content (not including fundamental) and RMS of the fundamental component. THD quantifies the effect of the odd order harmonics, where a large THD represents significant harmonic content with respect to the fundamental and low THD represents insignificant harmonic content with respect to the fundamental.

The output current THD of each simulation model is measured using Fourier analysis provided by LTspice for a variable load resistance. The optimum capacitance is kept constant while inductance is varied in accordance with the load to maintain a stable output frequency. The Fourier analysis is performed for 10 cycles and measured to 10 harmonics. The results are plotted in Figure 5-23.

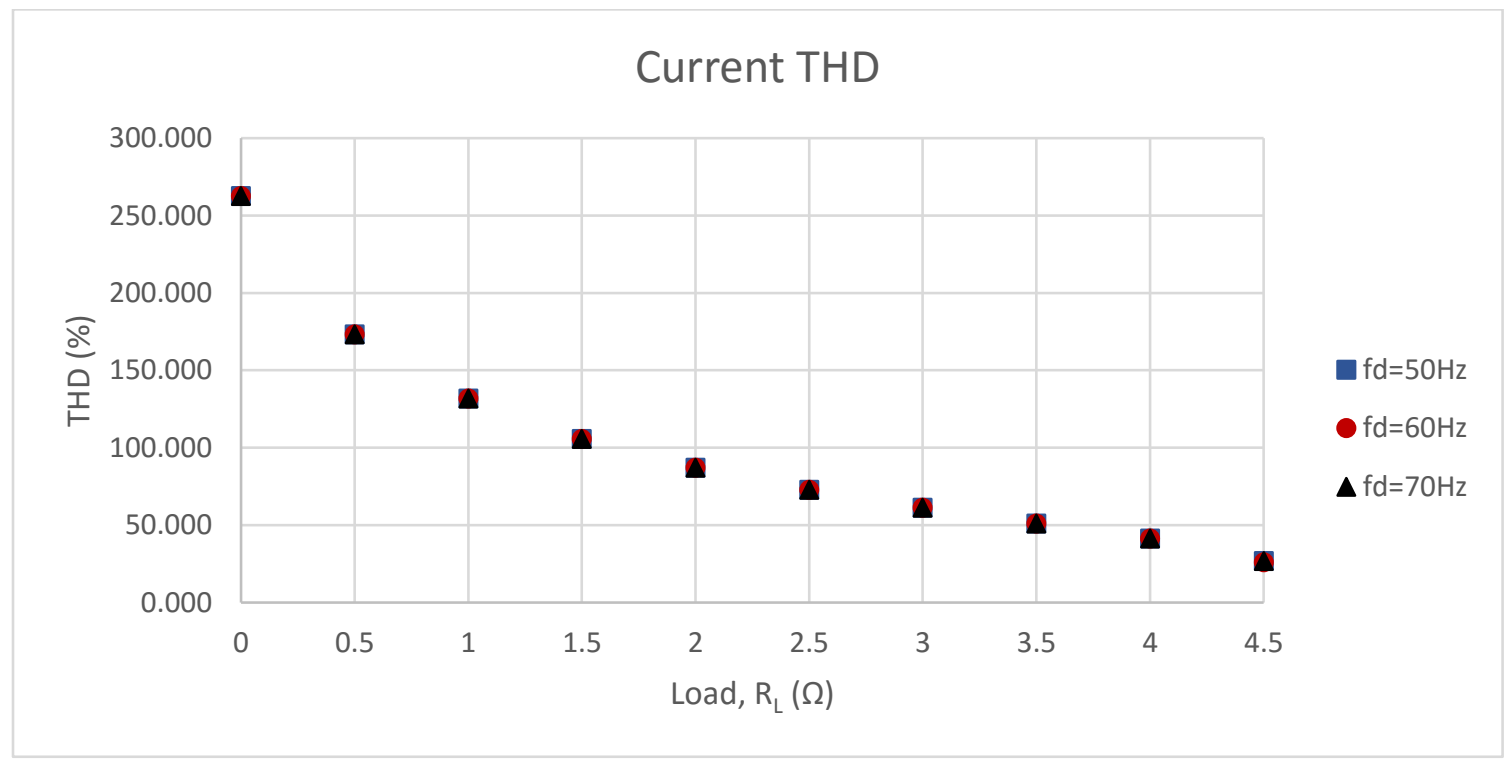

Figure 5-23: Output current THD vs. load for constant frequency using variable inductance 
The THD simulation results have corresponding performance for the three simulation models, as shown in Figure 5-23. This demonstrates the THD is dependent on the load and independent of the operating frequency. In addition, THD above $25 \%$ is observed for load resistances below the optimum design load resistance of $4.5 \Omega$. Once again, this suggest the variable inductance method for frequency stability is impractical, since frequency regulation is only attainable for load resistances below the designed load, which result in THD above $25 \%$.

Hardware results of THD are demonstrated in Figure 5-24: THD using variable inductance hardware results. This THD is equivalent to current THD, since this measurement is

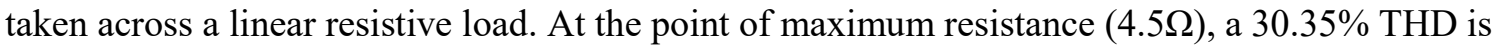
measured in hardware. Similarly, simulation demonstrates $26.83 \%$ at maximum resistance; thus corresponding to an error of $11.5 \%$. Note that a lower THD is observed for increasing inductance; which agrees to simulation results. Simulation results of Figure 5-23 correspond to higher portion of the nose curve demonstrated in Figure 5-24.

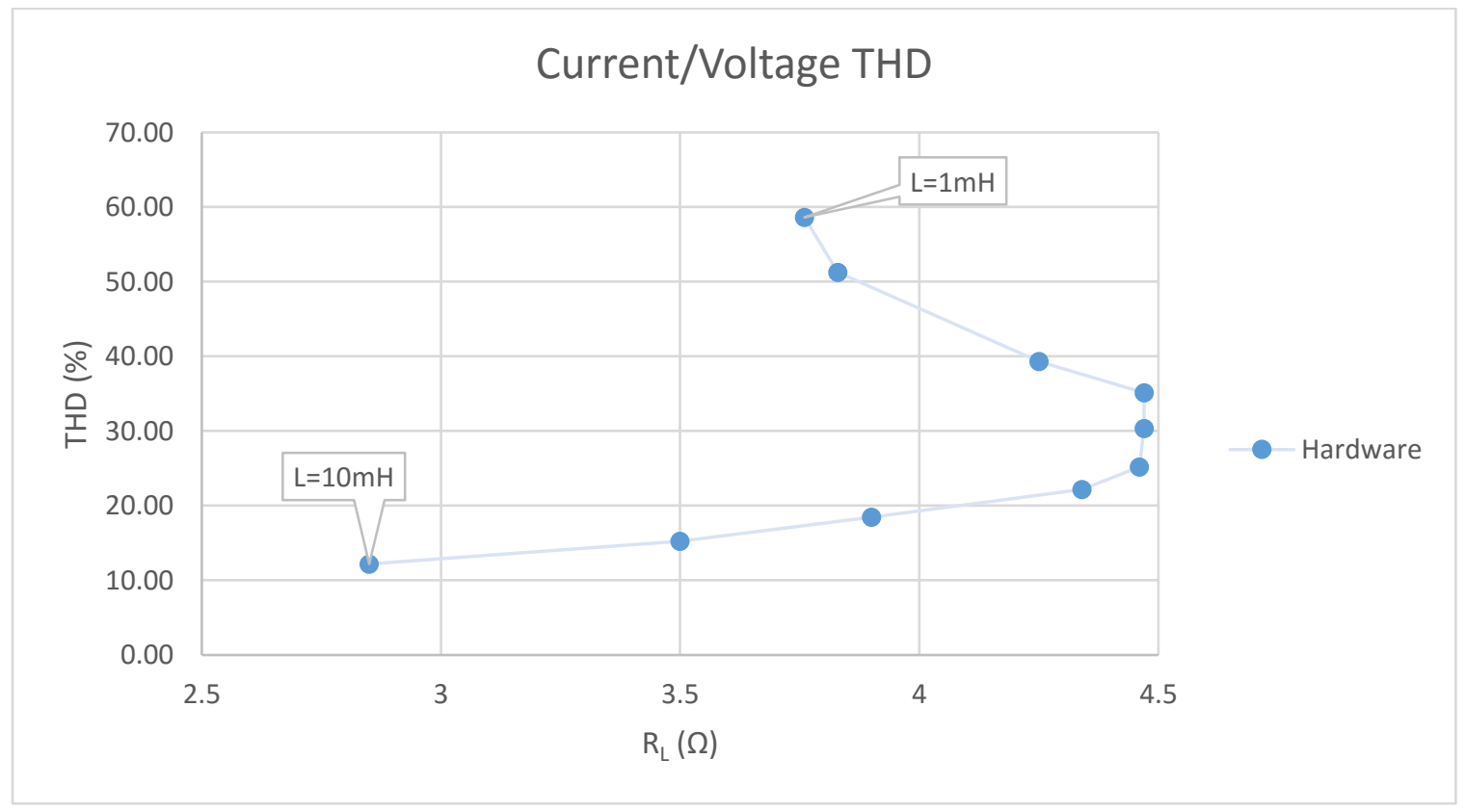

Figure 5-24: THD using variable inductance hardware results 


\subsection{Efficiency}

Equation (3-26) demonstrates the efficiency of the SLR inverter is strictly a function of the resistive elements in the series path. This assumes switching losses are neglected due to ZCS of the SLR inverter. To verify the ZCS and the efficiency model predicted by equation (3-26) an efficiency simulation is conducted for the three models. A total simulation time of $500 \mathrm{~ms}$ of steady state data is obtained to measure the efficiency for load resistances between $0 \Omega$ and $4.5 \Omega$. Frequency is kept constant by varying the inductance in accordance with the load resistance, while the optimum capacitance is fixed. Efficiency is measured by taking the ratio between the average power of the load and source. Simulation results are compared to the theoretical efficiency and plotted in Figure 5-25.

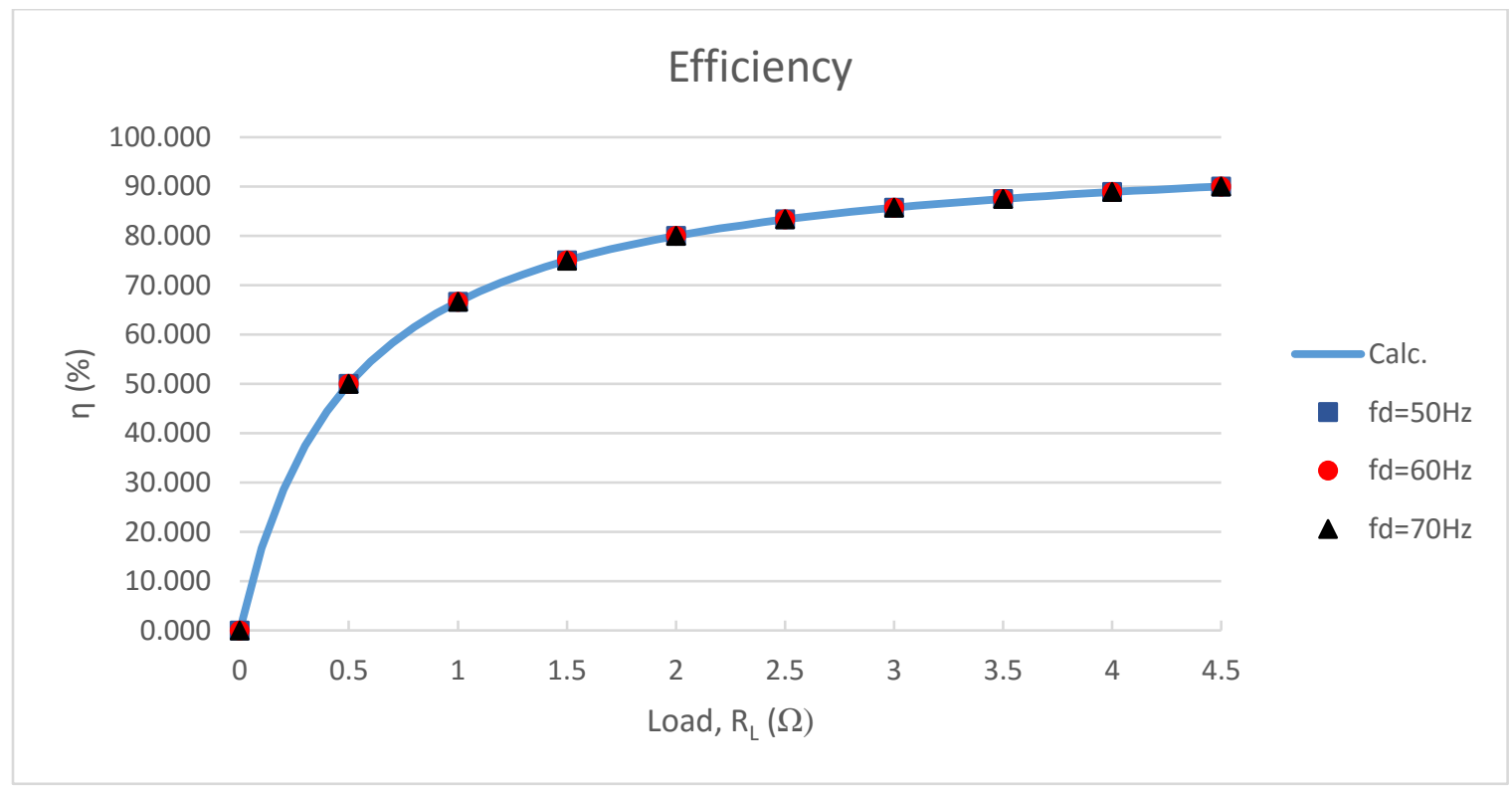

Figure 5-25: Efficiency vs. load resistance for constant frequency using variable inductance

Figure 5-25 demonstrates the agreement between theoretical (Calc.) and simulated efficiency. Equation (3-26) predicts efficiency increases as the ratio between the load and total path impedance increases; thus, higher efficiencies may be realized by either diminishing parasitic resistances, or increasing the load resistance. Frequency stability via variable inductance limits the load resistance and therefore the efficiency of the SLR inverter. 
Efficiency results obtained from hardware are demonstrated in Figure 5-26. Measured hardware efficiency is significantly less than simulation. This attributed to the increasing parasitic resistances as a function of increasing inductance. Simulation assumes series parasitic resistances remain constant as inductance increases; however, inductor series resistance increases as inductance increases, as demonstrated in Table 5-5. Additionally, hysteresis and eddy current losses are unaccounted for in simulation. These losses are proportional to the current through the inductor. To obtain an accurate model, inductor parasitic losses must be measured under loaded conditions.

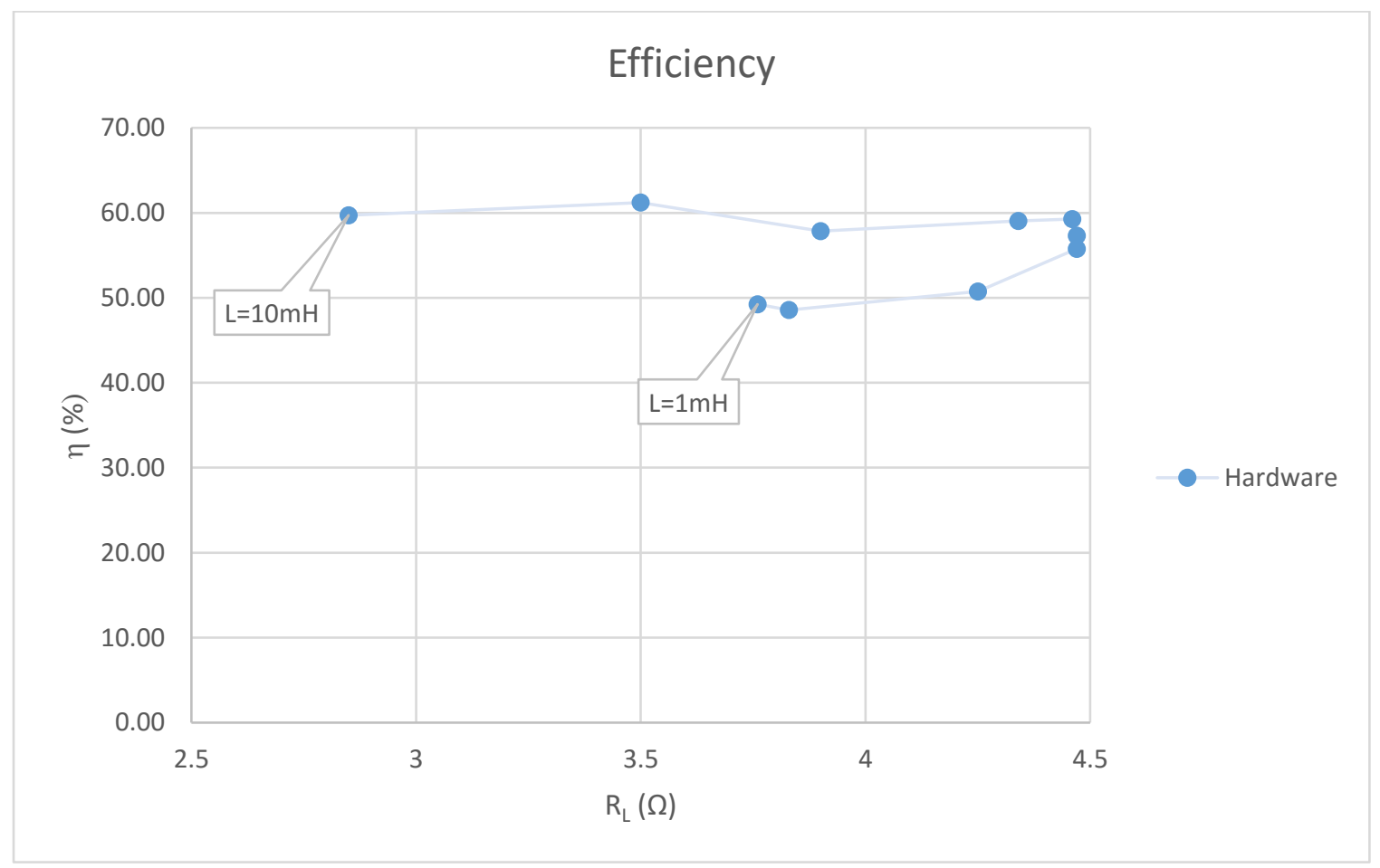

Figure 5-26: Hardware efficiency using variable inductance frequency stability 


\section{Chapter 6: Conclusion}

\subsection{Summary}

Results between simulation and hardware demonstrate accuracy of the mathematical model for inductances below the optimum operating point. Inductances beyond this range are impacted by capacitor initial conditions imposed on a cycle to cycle basis. Calculated current/voltage error between simulation and hardware results in a $2.5 \%$ maximum for inductances within the model range. Current regulation in hardware demonstrates a $70 \mathrm{~mA}_{\mathrm{RMS}}$ deviation for a $0.71 \Omega$ load change. The linear relationship between input voltage and output current may be utilized to achieve accurate current regulation.

Optimum inductor value selection is beneficial when designing for a load with greater load resistance than inductive parasitic resistances. It is evident from results that the inductor is a crucial component of the SLR inverter, since majority of losses are attributed to the inductor. This requires and inductor with large quality factor to obtain high efficiency. A significant efficiency error between simulation and hardware is attributed to inductor performance. Simulations assume constant parasitic resistances for variable inductance; however, hardware results demonstrate a significant dependence between inductance and parasitic components. Hysteretic and eddy current losses are unaccounted in both simulation and hardware. To obtain superior performance the designer must fully characterize the load and ensure a large ratio exists between load and parasitic resistances.

Hardware results show that increasing inductance reduces THD, but at the cost of increasing the size of magnetics. THD is a crucial component to sensitive loads, since harmonics with significant influence can cause instability in a system. Additionally, these harmonics can cause premature failure in mechanical equipment such as motors. To understand the impact of harmonics on motors, the inductive nature of the motor must first be characterized. If the inductance of the motor is significant, THD may be minimized. Additionally, a tunable magneto- 
electric inductor, [25], may be implemented in series with motor to achieve frequency stability. However, performance is limited by the load range, since loads greater than the reactance of the resonant capacitor result in switching losses.

Output frequency stability can only be maintained for loads below the reactance of the resonant capacitor. Loads beyond the reactance of the resonant capacitor result in switching losses. Thus, optimum inductor selection is based on a maximum load resistance, so as to achieve ZCS for loads below the maximum load resistance. However, efficiency and THD are negatively impacted for decreasing load resistance. The designer must seek a balance between efficiency and inductance based on the proposed design equations. For any case, the designer must ensure a full characterization of the inductor and load to determine appropriate design parameters.

\subsection{Potential Applications}

Harmonic distortion and low-efficiency of the hardware results suggest applications of the SLR inverter are limited. ZCS is the most attractive trait of the SLR inverter. Reduction in switching losses increases efficiency and reduces heating. THD is the greatest limiting feature of the SLR inverter, since pronounced harmonics of the fundamental frequency may cause mechanical failure in motors. Additionally, THD may cause significant electro-magnetic emissions; thus, compromising EMC requirement for high fidelity applications. Low power applications are more likely, since magnitude of harmonics are diminished, thus emit less electromagnetic radiation. Most AC applications drive motors, with the exception of lighting; thus, motors must be further investigated with the SLR inverter topology. From the data gathered in this thesis, high voltage low power motors are the most practical loads; however, greater research must be placed on load characterization to confirm a definitive application for SLR inverters. 


\subsection{Future Developments}

During the works of this thesis, additional methods of improving the SLR inverter were continually realized. This section focuses on demonstrating relevant improvements for the SLR inverter.

\subsubsection{State Space Model}

The mathematical model proposed in this thesis uses a transfer function method. From results, the model is only accurate for inductances below the optimum value. Inductances above the optimum value result in an error attributed to initial conditions imposed on the capacitor. A more accurate model may be developed using a state space method. The state space model allows for consideration of initial conditions; thus, a more accurate model may be achieved.

\subsubsection{Resonant Frequency Control}

Frequency control in the SLR inverter prototype is achieved using the IRS2153 selfoscillating half-bridge driver. This method requires manual adjustment of a potentiometer to regulate frequency. Alternatively, self-oscillating structures have been proposed in the literature [23]. This structure utilizes the resonant current through the inductor to actuate halfbridge switches; however, this is impractical for low frequency applications, due to the size of magnetic components. Similarly, a resonant current control strategy using current sensing eliminates the need for additional magnetic components and provides a self-resonating control.

\subsubsection{Output Voltage/Current Regulation}

The linear relationship between output voltage/current and supply voltage allow for regulation using a variable input voltage. Regulation may be implemented using a DC/DC converter that uses feedback from the output of the SLR inverter. An appropriate control 
strategy and DC/DC converter topology must be selected to optimize inverter load range. High voltage DC/DC converters are of preference due to ZCS of the SLR inverter.

\subsubsection{Load Characterization}

The gathered results demonstrate a great emphasis on load characterization. Motors have the greatest need for AC power; thus, investigation of suitable motor topologies is detrimental to SLR performance. Selected motors must be characterized experimentally to recognize SLR inverter performance based on the model developed in this thesis. Relevant parameters include operating inductance range, load, and losses. 


\section{REFERENCES}

[1] "Key World Energy Statistics 2015." International Energy Agency. OECD/IEA, Nov. 2015. Web. Mar. 2016.

[2] Hook, M, and X Tang. "Depletion of Fossil Fuels and Anthropogenic Climate Change-A Review." Energy Policy, 52 (2013): 797-809.

[3] Delucchi, Mark, and Mark Jacobson. "Meeting the World's Energy Needs Entirely with Wind, Water, and Solar Power." Bulletin of the Atomic Scientists, 69.4 (2013): $30-40$.

[4] Jacob, JA. "EPA Releases Final Clean Power Plan." Jama-journal of the American Medical Association, 314.12 (2015): 1216.

[5] United States. Energy Information Administration. Wind and Solar Data and Projections from the U.S. Energy Information Administration: Past Performance and Ongoing Enhancements. U.S. Department of Energy, 22 Mar. 2016. Web. Mar. 2016.

[6] Sarkar, Joydeep, and Pramod Yade. "Structuring DC Micro-Grid for Integrating Renewable Energy in a DC Load Dominant Electrical Environment." International Journal of Engineering Sciences \& Research Technology, 3.7 (2014): 609-613.

[7] Lotfi, Hossein, and Amin Khodaei. "AC Versus DC Microgrid Planning." IEEE Transactions on Smart Grid, (2015): 1.

[8] Sasidharan, N, MN Madhu, JG Singh, and W Ongsakul. "An Approach for an Efficient Hybrid AC/DC Solar Powered Homegrid System Based on the Load Characteristics of Home Appliances." Energy and Buildings, 108 (2015): 23-35. 
[9] Strunz, Kai, Ehsan Abbasi, and Duc Nguyen Huu. "DC Microgrid for Wind and Solar Power Integration." IEEE Journal of Emerging and Selected Topics in Power Electronics, 2.1 (2014): 115-126.

[10] Starke, M, Fangxing Li, L.M Tolbert, and B Ozpineci. "AC Vs. DC Distribution: Maximum Transfer Capability." 2008 IEEE Power and Energy Society General Meeting - Conversion and Delivery of Electrical Energy in the 21st Century, (2008): $1-6$.

[11] Owen, E.L. "History [Origin of the Inverter]." IEEE Industry Applications Magazine, 2.1 (1996): 64-66.

[12] Esram, T, and P.L Chapman. "Comparison of Photovoltaic Array Maximum Power Point Tracking Techniques." IEEE Transactions on Energy Conversion, 22.2 (2007): 439-449.

[13] Saidur, R, S Mekhilef, MB Ali, A Safari, and HA Mohammed. "Applications of Variable Speed Drive (VSD) in Electrical Motors Energy Savings." Renewable \& Sustainable Energy Reviews, 16.1 (2012): 543-550.

[14] Power Electronics Handbook. San Diego, Calif.: Academic Press, 2001.

[15] TT Electronics, Resistors for High Voltage Applications - Appl. Note 6488, online.

[16] A. Kumar and R. Gupta, "Capacitors voltage balancing in half bridge inverter for low switching frequency applications," Power Electronics, Drives and Energy Systems (PEDES) \& 2010 Power India, 2010 Joint International Conference, New Delhi, 2010, pp. 1-7.

[17] Colak, Ilhami, Ersan Kabalci, and Ramazan Bayindir. "Review of Multilevel Voltage Source Inverter Topologies and Control Schemes." Energy Conversion and Management, 52.2 (2011): 1114-1128. 
[18] Sandhu, Er. Mamatha, and Dr. Tilak Thakur. "Multilevel Inverters: Literature Survey - Topologies, Control Techniques \& Applications of Renewable Energy Sources Grid Integration." International Journal of Engineering Research and Applications, 4.3 (2014): 644-652.

[19] Gupta, Krishna Kumar, Alekh Ranjan, Pallavee Bhatnagar, Lalit Kumar Sahu, and Shailendra Jain. "Multilevel Inverter Topologies with Reduced Device Count: A Review." IEEE Transactions on Power Electronics, 31.1 (2016): 135-151.

[20] S.J. Finney, T.C. Green, and B.W. Williams. "Review of Resonant Link Topologies for Inverters." IEEE Proceedings-b Electric Power Applications, 140.2 (1993): 103114.

[21] Kumari, Veena, D.V. Bhaskar, N. Parida, and T. Maity. "Comparative Study of Multiple-output Series Resonant Inverters for IH Applications." 2015 International Conference on Circuits, Power and Computing Technologies [ICCPCT-2015], (2015): $1-7$.

[22] Cosby, M.C, and R.M. Nelms. "A Resonant Inverter for Electronic Ballast Applications." IEEE Transactions on Industrial Electronics, 41.4 (1994): 418-425.

[23] Chan, S.S.M, H.S.H Chung, and S.Y Hui. "Design and Analysis of an IC-less SelfOscillating Series Resonant Inverter for Dimmable Electronic Ballasts." IEEE Transactions on Power Electronics, 20.6 (2005): 1450-1458.

[24] Vroubel, M, Yan Zhuang, B Rejaei, and J.N Burghartz. "Integrated Tunable Magnetic RF Inductor." IEEE Electron Device Letters, 25.12 (2004): 787-789.

[25] Lin, Hwaider, Jing Lou, Yuan Gao, Ryusuke Hasegawa, Ming Liu, Brandon Howe, John Jones, Gail Brown, and Nian X Sun. "Voltage Tunable Magnetoelectric 
Inductors with Improved Operational Frequency and Quality Factor for Power Electronics." IEEE Transactions on Magnetics, 51.1 (2015): 1-5.

[26] Dong, Shuxiang, Xiaoxi Cui, and Guoxi Liu. "A Tunable Ring-type Magneto-electric Inductor." Journal of Applied Physics, 108.9 (2010): 094106-094106-4.

[27] Kasturi, R. "An Analysis of Series Inverter Circuits." IEEE Transactions on Industrial Electronics and Control Instrumentation, IECI-22.4 (1975): 515-519.

[28] Stewart, James. "Reference Pages." Calculus: Early Transcendentals. 8th ed. Salt Lake City: Brooks Cole, 2015. 8. Print.

[29] International Rectifier Datasheet. IRFB7540 - Hexfet Power Mosfet (E1 Segundo, CA, 2014) 15 May. $2016<$ http://www.irf.com/productinfo/datasheets/data/irfs7540pbf.pdf $>$

[30] International Rectifier Datasheet. IRS2153 - Self-oscillating half-bridge driver IC (El Segundo, CA, 2014) 15 May. $2016<\underline{\text { http://www.irf.com/product- }}$ info/datasheets/data/irs2153d.pdf $>$

[31] International Rectifier Application Note. AN-978 - HV Floating MOS-Gate Driver ICs (El Segundo, CA, 2007) 15 May. $2016<\underline{\text { http://www.infineon.com/dgdl/an- }}$ 978.pdf?fileId $=5546 \mathrm{~d} 462533600 \mathrm{a} 40153559 \mathrm{f} 7 \mathrm{cf} 21200>$ 


\section{Appendix A: LTspice Half-Bridge Controller Module}

Author: K. M.

Source: http://home.scarlet.be/nap0/ltspice/index.html

Date Accessed: January 12, 2016

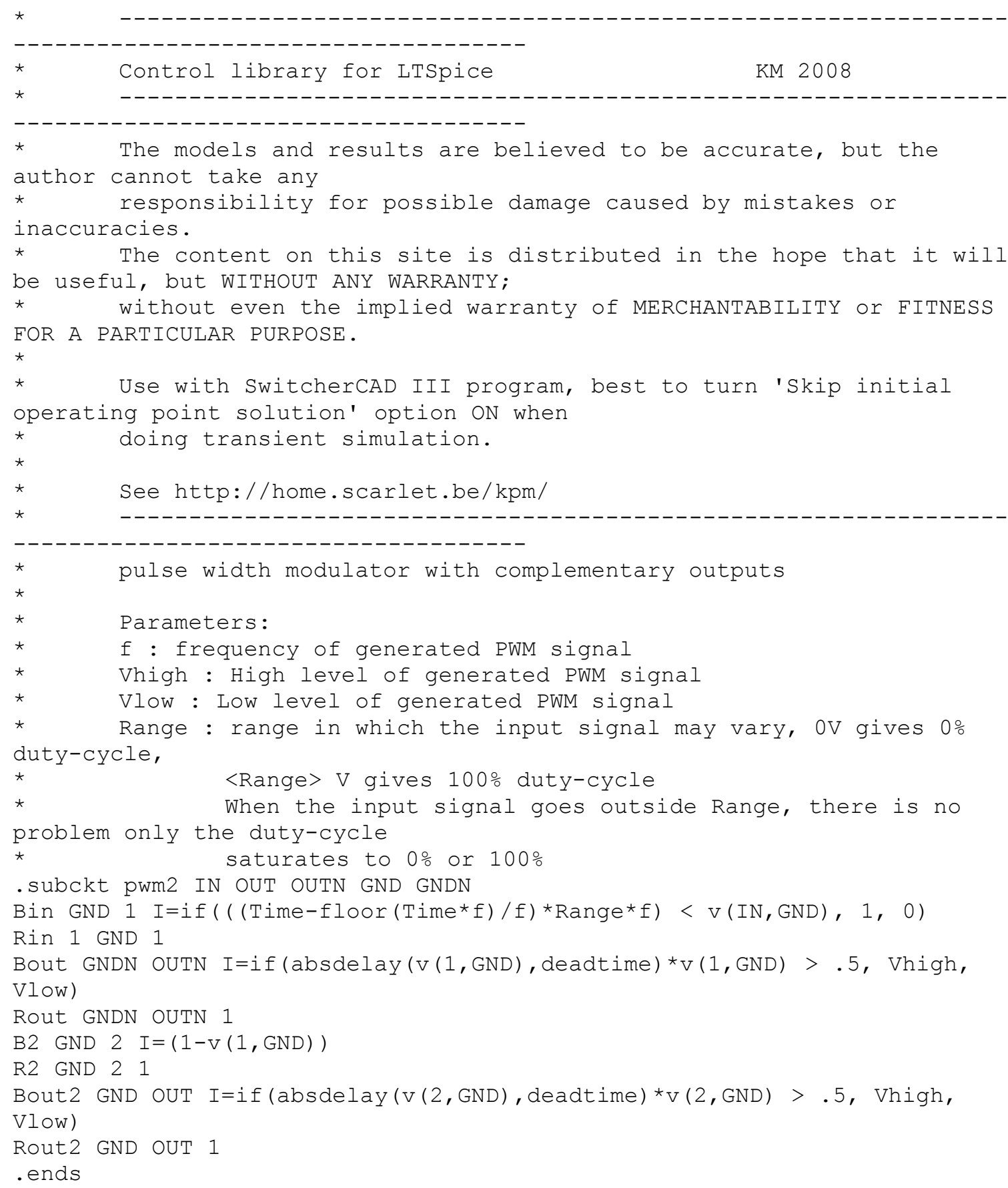

